\title{
Rectal cancer imaging : staging and restaging
}

Citation for published version (APA):

Vliegen, R. F. A. (2008). Rectal cancer imaging : staging and restaging. [Doctoral Thesis, Maastricht University]. Datawyse / Universitaire Pers Maastricht. https://doi.org/10.26481/dis.20080618rv

Document status and date:

Published: 01/01/2008

DOI:

10.26481/dis.20080618rv

Document Version:

Publisher's PDF, also known as Version of record

\section{Please check the document version of this publication:}

- A submitted manuscript is the version of the article upon submission and before peer-review. There can be important differences between the submitted version and the official published version of record.

People interested in the research are advised to contact the author for the final version of the publication, or visit the DOI to the publisher's website.

- The final author version and the galley proof are versions of the publication after peer review.

- The final published version features the final layout of the paper including the volume, issue and page numbers.

Link to publication

\footnotetext{
General rights rights.

- You may freely distribute the URL identifying the publication in the public portal. please follow below link for the End User Agreement:

www.umlib.nl/taverne-license

Take down policy

If you believe that this document breaches copyright please contact us at:

repository@maastrichtuniversity.nl

providing details and we will investigate your claim.
}

Copyright and moral rights for the publications made accessible in the public portal are retained by the authors and/or other copyright owners and it is a condition of accessing publications that users recognise and abide by the legal requirements associated with these

- Users may download and print one copy of any publication from the public portal for the purpose of private study or research.

- You may not further distribute the material or use it for any profit-making activity or commercial gain

If the publication is distributed under the terms of Article $25 \mathrm{fa}$ of the Dutch Copyright Act, indicated by the "Taverne" license above, 
RECTAL CANCER IMAGING

staging and restaging 
ISBN: 9789052787251

Copyright $\odot$ R.F.A. Vliegen, Heerlen 2008. All rights reserved.

The copyright of articles that have been published or accepted for publication has been transferred to the respective journals.

Layout: I. Kengen

Printed by: Datawyse | Universitaire Pers Maastricht 


\title{
RECTAL CANCER IMAGING
}

\section{staging and restaging}

\author{
Proefschrift
}

ter verkrijging van de graad van doctor aan de Universiteit Maastricht, op gezag van de Rector Magnificus Prof. mr. G.P.M.F. Mols, volgens het besluit van het College van Decanen, in het openbaar te verdedigen

op woensdag 18 juni 2008 om 12.00 uur

door

Roy Frans Arnold Vliegen

Geboren op 6 december 1969 te Heerlen

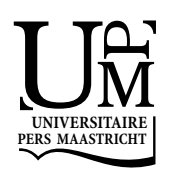


Promotores:

Prof. Dr. R.G.H. Beets-Tan

Prof. dr. J.M.A. van Engelshoven

Copromotores:

Dr. G.L. Beets

Beoordelingscommissie:

Prof. dr. P.B. Soeters (voorzitter)

Prof. dr. ir. P van den Brandt

Prof. dr. Ph. Lambin

Prof. dr. J. Stoker (Academisch Medisch Centrum, Universiteit van Amsterdam)

Prof. dr. V.C. Tjan-Heijnen 
Aan mijn vrouw Andrea 



\section{Contents}

Chapter 1 Introduction and aim of the thesis

Chapter 2 Magnetic Resonance Imaging of rectal cancer: what radiation oncologists need to know

Frontiers Radiation Therapy Oncology 2004; 38:1-12.

Chapter 3 Rectal cancer: MR Imaging in local staging-Is gadoliniumbased contrast material helpful?

Radiology 2005; 234:179-188.

Chapter 4 The accuracy of Multi-detector row CT for the assessment of tumor invasion of the mesorectal fascia in primary rectal cancer

Abdominal Imaging 2008 (in press)

Chapter 5 The accuracy of MRI in predicting mesorectal fascia invasion after neo-adjuvant chemoradiation for locally advanced rectal cancer

Radiology 2008; 246:454-62.

Chapter 6 How accurate can sequential FDG-PET/CT predict tumor regression from the mesorectal fascia in locally advanced rectal cancer treated with neo-adjuvant chemoradiation therapy?

Submitted for publication.

Chapter 7 General discussion

Chapter 8 Summary

Samenvatting

Dankwoord

Curriculum vitae 



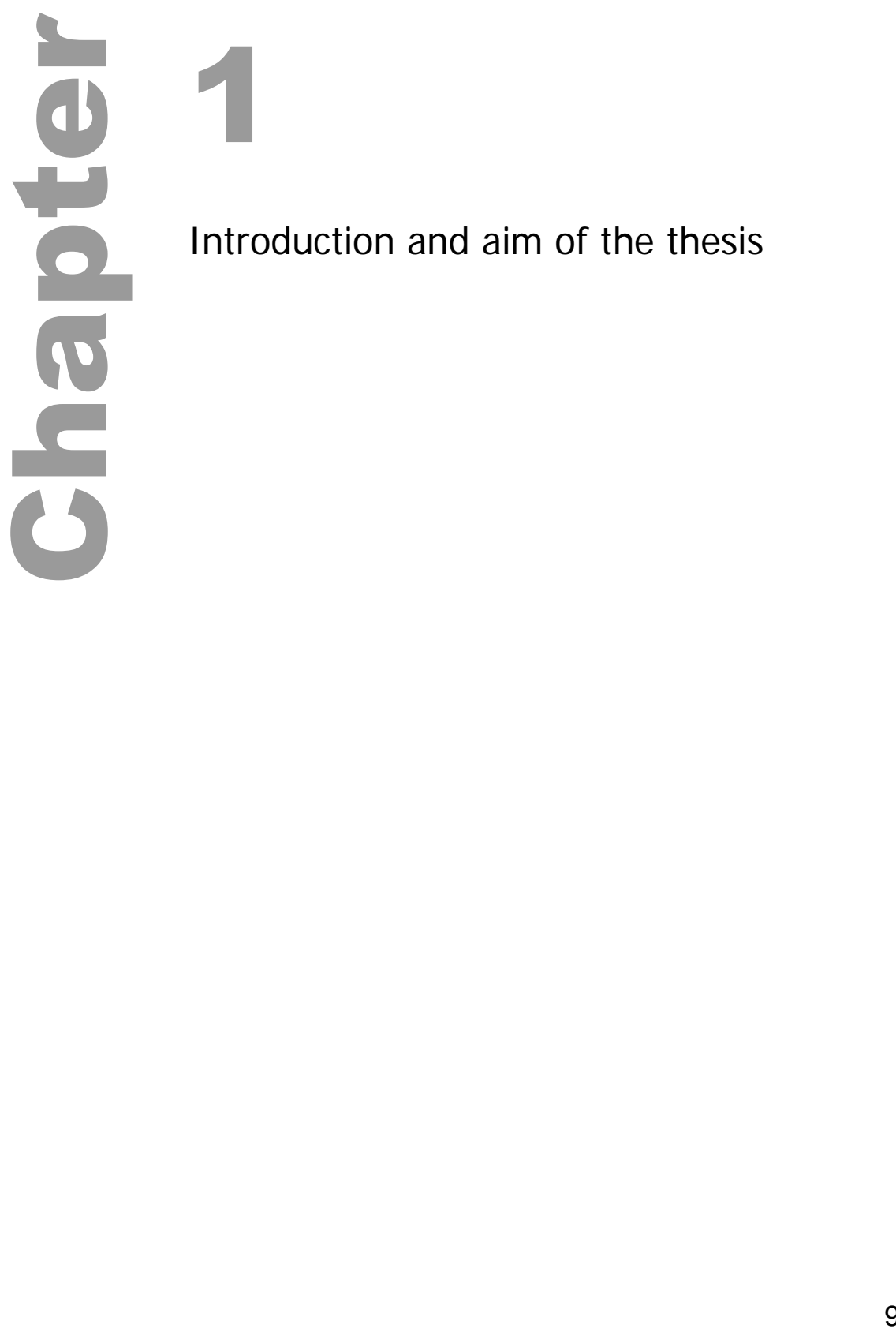




\section{Introduction}

Colorectal cancer is a major health problem with an incidence of 10000 and a mortality rate of 4500 patients a year in The Netherlands (1). One-third of all colorectal cancers occur in the rectosigmoid or rectal region.

The primary treatment of rectal cancer has been surgery, with a mainly blunt dissection technique of the rectum. This technique resulted in a high local tumor recurrence (up to $30 \%$ ) because of the incomplete removal of the later tumor spread (2-5). Since then, much effort has been focussed on the local tumor control. First, the surgical technique has been improved by the introduction of the surgical concept of total mesorectal excision (figure 1). This technique, propagated by Heald resulted in a much lower local recurrence rate (reported below 10\%), and therefore it is currently considered as standard for non locally advanced rectal cancer (6). In addition, both a short course of preoperative (neo-adjuvant) radiotherapy (5x5 Gy) and a combination of long courses of preoperative radiation therapy and chemotherapy have been shown to further improve local tumor control in addition to optimized surgical techniques (7-12).

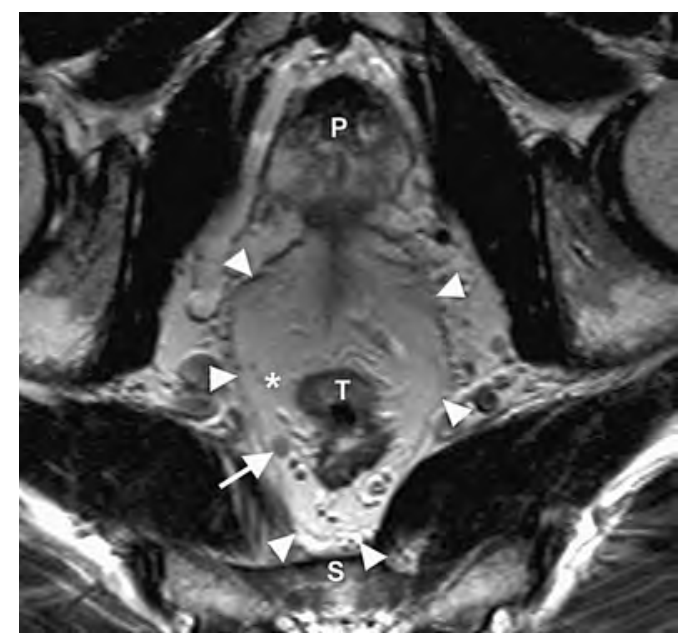

Fig. 1. Transverse Magnetic Resonance Image illustrating the principles of total mesorectal excision surgery (TME) which is the current standard treatment of tumors confined to the mesorectal compartment (non locally advanced cancer). The rectum including the tumor $(T)$ and surrounding mesorectal=perirectal fat tissue (asterisk) is removed by sharp dissection along the mesorectal fascia (arrowheads). The mesorectal fascia defines the surgical resection plane in TME (arrow=lymph node; $p=$ prostate; $s=$ sacrum).

However, the downside of neo-adjuvant treatment is not just extra cost, but also morbidity (13). Therefore, there is a current trend to individualize the treatment according to the risk for local recurrence instead of a uniform treatment. Using neo-adjuvant therapy schedules in patients who need it most 
improves the therapeutic ratio and minimizes the associated morbidity. The most important risk factors for a local recurrence are the distance of the tumor to the mesorectal fascia, distal tumors and metastatic lymph node disease (14-16). The current trend in the treatment of non locally advanced rectal cancer is the application of short courses of neo-adjuvant radiation therapy (5x5gy) followed by TME surgery $(7,17)$. Whereas long courses of chemoradiation therapy are reserved for patients showing previous mentioned risk factors for local recurrence, followed by often more extensive surgical resections depending on the tumor shrinkage effect of neo-adjuvant therapy (8-10,15,17-19).

When considering individualized treatment strategies, there is a pivotal role of imaging. It provides important information to stratify rectal cancer patients into different risk groups for local recurrence (19,20-23). Presently, there are different imaging techniques available each with their strengths as well as shortcomings with a continuous improvement of each technique.

Endorectal ultrasound (EUS) is the prefered imaging technique for the evaluation of superficial (confined to the rectal wall) rectal tumors when local excision is considered only in selected cases $(17,20,24)$. The close position of the image probe to the rectal wall provides detailed anatomical information of the individual rectal wall layers but the image quality is insufficient for deeper pelvic structures because of signal drop off. Combined with the high interobserver disagreement of this technique, it is not suitable for the evaluation of more advanced rectal cancer and its relationship to clinical important structures like the mesorectal fascia and pelvic organs (20,24-25).

High resolution Magnetic Resonance Imaging (MRI) has gained worldwide acceptance and is presently established as the selection tool of choice for the determination of the most appropriate treatment regime of rectal cancer patients due to its proven high effectiveness to identify patients with a close or tumor invaded circumferential resection margin (17,20-23,26-27). The standard MR imaging protocol of rectal cancer consists of T2-weighted Fast Spin Echo (FSE) MR images acquired with an external phased array coil technique $(23,27-28)$. Differences in opinion exist regarding the application of contrast enhanced sequences (29-32). Studies evaluating their value are scarce and non of these studies have evaluated the value of these sequences for the prediction of tumor invasion of the mesorectal fascia (29-32). It is evident that when contrast enhanced sequences can be omitted, it would save imaging time, reduce costs and prevent potential complications.

For years, Comuted Tomography (CT) could not compete with the equisite anatomical detail provided by MRI and therefore it played a non important role in the local tumor staging of rectal cancer (17). Recently, improvements of the image quality of CT have been achieved by the invention of Multi-Detector row techniques. The main advantage of Multi-Detector row CT (MDCT) is the capability to perform a quick one stop shop examination of the 
whole body and therefore it may enable to make an integral treatment plan. The local staging capabilities of MDCT have been addressed in a few papers (33-36). These studies have shown high accuracies (86-95\%) for the prediction of $\mathrm{T}$ stage which is encouraging but rather irrelevant for daily practice. Instead, the question whether MDCT is as good as MRI for predicting the tumor relationship to the clinical important mesorectal fascia needs to be answered.

The introduction of modern chemoradiation regimens in the treatment of locally advanced rectal cancer have resulted in improved response rates which have created potential new treatment options (37). Whereas previously a complete response was sporadically observed, some regimens now show complete response rates of $20-30 \%$ (37-39). Although no doubt this is beneficial, it also brings new questions and new dilemmas. After a good response for a locally advanced tumor, is an extensive resection still required? Do the previously involved organs need to be resected? And more controversially, can a local excision be performed when the tumor appears limited to the bowel wall after chemoradiation, or is there a complete response and can surgery be withheld altogether? (40-41). There is a clear need to assess the response after chemoradiation, and both MRI and Positron Emission Tomography-Computed Tomography (PET-CT) could be of use. Upto now, studies on the MRI prediction of tumor response are scarce (42-44). They have mainly focussed on T-staging and not on more relevant surgical questions how good they perform for the prediction of tumor regression from the mesorectal fascia. PET studies have mainly focussed on the prediction of pathological tumor response and not on the assessment of anatomical tumor regression which is obvious important information when more conservative resections are contemplated (45-49).

\section{Objectives of this thesis}

1. To establish the optimal Magnetic Resonance Imaging (MRI) protocol for rectal cancer staging.

2. To evaluate the role of Multi-Detector row Computed Tomography (MDCT) as an alternative to MRI for local staging of rectal cancer.

3. To determine the role of MRI for assessing tumor response after neoadjuvant chemoradiation therapy.

4. To evaluate the role of Positron Emission Tomography-Computed Tomography (PET-CT) for assessing tumor response after neo-adjuvant chemoradiation therapy.

\section{Outline of this thesis}

Chapter 2 gives a general overview of the role, performance, limitations and technique of MRI in the diagnostic work-up of rectal cancer patients. 
Chapter 3 evaluates the additional value of gadolinium enhanced Magnetic Resonance Imaging (MRI) sequences as a supplement to T2-weighted Fast Spin Echo MR images for the purpose of rectal cancer staging and defines an optimized MR imaging protocol.

Chapter 4 assesses the accuracy of Multi-Detector row CT (MDCT) for the identification of locally advanced rectal cancer with tumor invasion of the mesorectal fascia.

Chapter 5 evaluates the accuracy of postchemoradiation MRI for the prediction of tumor invasion of the mesorectal fascia in locally advanced primary rectal cancer treated with neo-adjuvant chemoradiation therapy.

Chapter 6 assesses the value of Positron Emission Tomography-Computed Tomography (PET-CT) for the prediction of tumor regression from the mesorectal fascia in locally advanced rectal cancer treated with neo-adjuvant chemoradiation therapy.

Chapter 7 consists of the general discussion of this thesis, conclusions and recommendations.

Chapter 8 includes the summary of this thesis.

\section{References}

1. KWF 2007: www.KWFkankerbestrijding nl

2. Sagar $\mathrm{P}$, Pemberton J. Surgical management of locally recurrent rectal cancer. $\mathrm{Br} \mathrm{J}$ Surg 1996; 83:293-304.

3. Fielding L, Stewart-Brown S, Dudley $\mathrm{H}$. Surgeon-related variables and the clinical trial. Lancet 1978; 2:778-779.

4. Quirke P, Durdey P, Dixon M, et al. Local recurrence of rectal adenocarcinoma due to inadequate surgical resection: histopathological study of lateral tumour spread and surgical excision. Lancet 1986; 2:996-999.

5. Quirke P, Dixon M. The prediction of local recurrence in rectal adenocarcinoma by histopathological examination. Int J Colorectal Dis 1988; 3:127-131.

6. Heald R, Ryall R. Recurrence and survival after total mesorectal excision for rectal cancer. Lancet 1986; 1:1479-1482.

7. Kapiteijn E, Marijnen C, Nagtegaal I, et al. Preoperative radiotherapy combined with total mesorectal excision for resectable rectal cancer. N Engl J Med 2001; 345:638-646.

8. Sauer $R$, Beckers $H$, Hohenberger $W$, et al. Preoperative vs postoperative chemoradiotherapy for rectal cancer. N Engl J Med 2004; 351:1731-1740.

9. Bosset J, Collette L, Calais G, et al. Chemotherapy with preoperative radiotherapy in rectal cancer. N Engl J Med 2006; 355:1114-1123.

10. Gerard J, Conroy T, Bonnetain F, et al. Preoperative radiotherapy with or without concurrent fluorouracil and leucovorin in T3-4 rectal cancers: results of FFCD 9203. J Clin Oncol 2006; 24:4620-4625. 
11. Improved survival with preoperative radiotherapy in resectable rectal cancer: Swedish Rectal Cancer Trial. N Engl J Med 1997; 336:980-987.

12. Colorectal Cancer Collaborative Group. Adjuvant radiotherapy for rectal cancer: a systematic overview of 8,507 patients from 22 randomised trials. Lancet 2001; 358:12911304.

13. Birgisson $\mathrm{H}$, Pahlman L, Gunnarsson U, et al. Late adverse effects of radiation therapy for rectal cancer-a systemic overview. Acta Oncol 2007; 46:504-516.

14. Moran $M$, James $E$, Rothenberger $D$, et al. Prognostic value of positive lymph nodes in rectal cancer. Dis Colon Rectum 1992; 35:579-581.

15. Nagtegaal I, Marijnen C, Kranenbarg E, et al. Circumferential margin involvement is still an important predictor of local recurrence in rectal carcinoma: not one millimeter but two millimeters is the limit. Am J Surg Pathol 2002; 26:350-357.

16. den Dulk M, Marijnen $\mathrm{C}$, Putter $\mathrm{H}$, et al. Risk factors for adverse outcome in patients with rectal cancer treated with an abdominal resection in the total mesorectal excision trial. Ann Surg 2007; 246:83-90.

17. Valentini V, Glimelius B, Minsky B, et al. The multidisciplinary rectal cancer treatment: main convergences, controversial aspects and investigational areas which support the need for an European consensus. Radiother Oncol 2005; 76:241-250.

18. Chau I, Brown G, Cunningham D, et al. Neoadjuvant capecitabine and oxaliplatin followed by synchronous chemoradiation and total mesorectal excision in magnetic resonance imaging defined poor risk rectal cancer. J Clin Oncol 2006; 24:668-674.

19. Wiggers T. Staging of rectal cancer. Br J Surg 2003; 90:895-896.

20. Engelen S, Beets G, Beets-Tan R. Role of preoperative local and distant staging in rectal cancer. Onkologie 2007; 30:141-145.

21. Lahaye $M$, Engelen $S$, Nelemans $P$, et al. Imaging for predicting the risk factors-the circumferential resection margin and nodal disease-of local recurrence in rectal cancer: a meta-analysis. Semin Ultrasound CT MR 2005; 26:259-268.

22. Beets-Tan R, Beets $G$. Rectal cancer: how accurate can imaging predict the $T$ stage and the circumferential resection margin? Int J Colorectal Dis 2003; 18:385-391.

23. MERCURY study group. Diagnostic accuracy of preoperative magnetic resonance imaging in predicting curative resection of rectal cancer: prospective observational study. BMJ 2006; 333:779-790.

24. Glancy D, Pullyblank A, Thomas M, et al. The role of endoanal ultrasound scanning (EUS) in selecting patients suitable for resection by transanal endoscopic microsurgery (TEM). Colorectal Dis 2005; 7:148-150.

25. Burtin $P$, Rabot $A$, Heresbach $D$, et al. Interobserver agreement in the staging of rectal cancer using endoscopic ultrasonography. Endoscopy 1997; 29:620-625.

26. Beets-Tan $R$, Beets $G$, Vliegen $R$, et al. Accuracy of magnetic resonance imaging in prediction of tumour-free resection margin in rectal cancer surgery. The Lancet 2001; 357:497-504.

27. Brown G, Radcliffe A, Newcombe R, et al. Preoperative assessment of prognostic factors in rectal cancer using high-resolution magnetic resonance imaging. $\mathrm{Br} J$ Surg 2003; 90:355-364.

28. Brown G, Richards C, Newcombe R, et al. Rectal carcinoma: thin-section MR imaging for staging in 28 patients. Radiology 1999; 211:215-222.

29. Okizuka H, Sugimura K, Yoshizako $T$, et al. Rectal carcinoma: prospective comparison of conventional and gadopentetate dimeglumine enhanced fat-suppressed MR imaging. J Magn Reson Imaging 1996; 6:465-471. 
30. Maier A, Kersting-Sommerhoff B, Reeders J, et al. Staging of rectal cancer by doublecontrast MR imaging using the rectally administered superparamagnetic iron oxide contrast agent ferristine and IV gadodiamide injection: results of a multicenter phase II trial. J Magn Reson Imaging 2000; 12:651-660.

31. Vogl T, Pegios W, Mack M, et al. Accuracy of staging rectal tumors with contrastenhanced transrectal MR imaging. Am J Roentgenol 1997; 168:1427-1434.

32. Wallengren N, Holtås S, Andrén-Sandberg $\AA$, et al. Rectal carcinoma: double contrast MR imaging for preoperative staging. Radiology 2000; 215:108-114.

33. Kulinna C, Eibel R, Matzek W, et al. Staging of rectal cancer: diagnostic potential of multiplanar reconstructions with MDCT. Am J Roentgenol 2004; 183:421-427.

34. Matsuoka H, Nakamura A, Masaki $\mathrm{T}$, et al. A prospective comparison between multidetector-row computed tomography and magnetic resonance imaging in the preoperative evaluation of rectal carcinoma. Am J Surg 2003; 185:556-559.

35. Kulinna C, Scheidler J, Strauss T, et al. Local staging of rectal cancer: assessment with double-contrast multi-slice computed tomography and transrectal ultrasound. J Comput Assist Tomogr 2004; 28:123-130.

36. Sinha $R$,Verma $R$, Rajesh $A$, et al. Diagnostic value of multi-detector row $C T$ in rectal cancer staging: comparison of multiplanar and axial images with histopathology. Clin Radiol 2006; 61:924-931.

37. Hartley A, Ho K, McConkey $\mathrm{C}$, et al. Pathological complete response following preoperative chemoradiotherapy in rectal cancer: analysis of phase II/III trials. $\mathrm{Br} J$ of Radiol 2005; 78:934-938.

38. Tobin R, Mohiuddin M, Marks G. Preoperative irradiation for cancer of the rectum with extrarectal fixation. Int J Radiat Oncol Biol Phys 1991; 21:1127-1132.

39. Read $T$, Olajungu $A$, Ogunbiyi $O$, et al. Neoadjuvant external beam radiation and proctectomy for adenocarcinoma of the rectum. Dis Colon Rectum 2001; 44:1778-1790.

40. Bonnen M, Crane C, Vauthey J, et al. Long-term results using local excision after preoperative chemoradiation among selected T3 cancer patients. Int J Radiat Oncol Biol Phys 2004; 60:1098-1105.

41. Habr-Gama A, Perez R, Nadalin W, et al. Operative versus nonoperative treatment for stage 0 distal rectal cancer following chemoradiation therapy: long-term results. Ann Surg 2004; 240:711-717.

42. Kuo L, Chern M, Tsou M, et al. Interpretation of magnetic resonance imaging for locally advanced rectal carcinoma after preoperative chemoradiation therapy. Dis Colon Rectum 2005; 48:23-28.

43. Hoffmann K, Rau B, Wust $P$, et al. Restaging of locally advanced carcinoma of the rectum with MR imaging after preoperative radio-chemotherapy plus regional hyperthermia. Coloproctology 2002; 24:253-261.

44. Chen $C$, Lee $R$, Lin J, et al. How accurate is magnetic resonance imaging in restaging rectal cancer in patients receiving preoperative combined chemoradiotherapy? Dis Colon Rectum 2004; 48:722-728.

45. Capirci C, Rampin L, Erba P, et al. Sequential FDG-PET-CT reliably predicts response of

46. locally advanced rectal cancer to neo-adjuvant chemo-radiation therapy. Eur J Nucl Med Mol Imaging 2007; 34:1583-1593.

47. Capirci C, Rubello D, Chierichetti $F$, et al. Restaging after neoadjuvant chemoradiotherapy for rectal adenocarcinoma : role of F18-FDG PET. Biomed Pharmacother 2004; 58:451457.

48. Cascani G, Avallone A, Delrio P, et al. 18F-FDG PET is an early predictor of pathologic tumor response to preoperative radiochemotherapy in locally advanced rectal cancer. J Nucl Med 2006; 47:1241-1248.

49. Melton $\mathrm{G}$, Lavely W, Jacene $\mathrm{H}$, et al. Efficacy of preoperative combined 
50. 18-Fluorodeoxyglucose Positron Emission Tomography and Computed Tomography for assessing primary rectal cancer response to neoadjuvant therapy. J Gastrointest Surg 2007; 11:961-969.

51. Young $H$, Baum $R$, Cremerius $U$, et al. Measurement of clinical and subclinical tumour response using [18F]-fluorodeoxyglucose and positron emission tomography: review and 1999 EORTC recommendations. Eur J Cancer 1999; 35:1773-1782. 


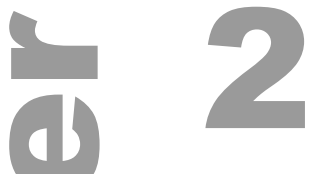

Magnetic Resonance Imaging of rectal cancer: what radiation oncologists need to know

RG Beets-Tan, RF Vliegen, GL Beets.

Frontiers Radiation Therapy Oncology 2004; 38:1-12. 


\section{Introduction}

Rectal cancer carries a poor prognosis because of the risk of both metastases and local recurrences. Although local recurrences have a small impact on survival rates, they have a profound impact on the quality of life. A local recurrence is often debilitating because of severe pain and immobility, and prolonged and multiple hospital admissions for surgery, radiation and chemotherapy. Attention has therefore mainly been directed towards defining the best treatment strategy for the primary tumor in order to obtain optimal local control, combining radiation therapy (I-4) with optimal surgery, a total mesorectal excision (TME) (5) (figure 1).

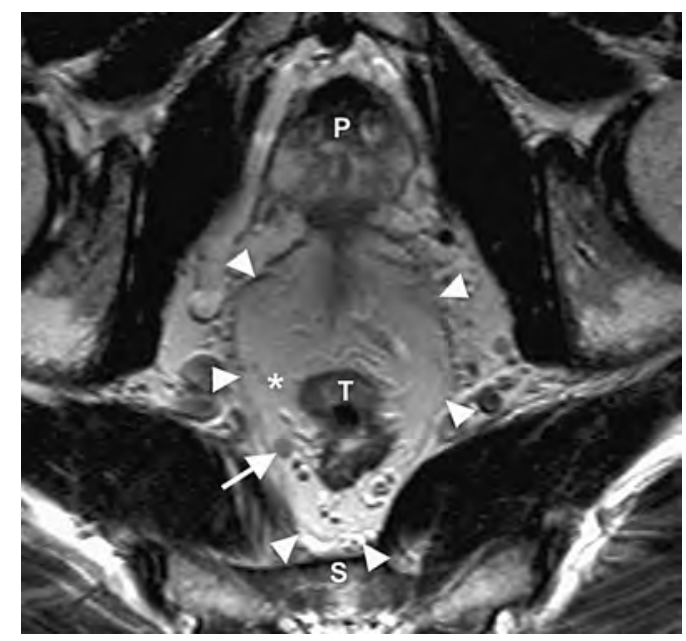

Fig. 1. Total mesorectal excision (TME). Axial T2W TSE MR image of a patient with rectal cancer clearly shows the mesorectal fascia (arrowheads) enveloping the mesorectum. The mesorectum is an anatomic compartment that comprises the rectum ( $T$ = tumor in rectum), the mesorectal fat (asterisk), blood vessels, nerves and perirectal lymph nodes (arrow). TME surgery removes the mesorectum by sharp dissection along the mesorectal fascia. $\mathrm{P}=$ Prostate; $\mathrm{S}=$ sacrum.

Because high-risk patients benefit from extensive neoadjuvant treatment (6), imaging can play an important role in the preoperative identification of these patients. High-resolution Magnetic Resonance Imaging (MRI) has recently been reported to he a reliable tool for the preoperative identification of the circumferential resection margin at TME $(7,8)$, an important prognostic indicator for local recurrences. MRI has also been reported to be superior to Computed Tomography (CT) for the preoperative assessment of tumor invasion in surrounding structures $(9,10)$. MRI is therefore more frequently being applied as a routine investigation in the preoperative work up of patients with rectal cancer. Clinicians dealing with rectal cancer patients are 
nowadays confronted not only with MR images of rectal tumors but also with the complex MR images of irradiated rectal cancer.

This essay provides an overview of significant MR findings in rectal cancer and illustrates some of the interpretation difficulties in MR images of irradiated rectal cancer.

\section{MRI techniques and T staging accuracies}

The successful introduction of MRI in imaging of pelvic diseases and the numerous reports on the high performance of MRI have over recent years caused MRI to gradually replace CT in preoperative staging of rectal cancer. Initial MRI studies used the body coil. Because conventional body coil techniques showed a resolution that was still insufficient to differentiate the individual layers of the rectal wall, overall accuracies reported for body coil MRI have not been any better than those reported for $\mathrm{CT}$ with figures ranging from 59 to $88 \%$ (11-17).

With the introduction of endoluminal coils, image resolution improved and detailed evaluation of the layers of the rectal wall was feasible (18). This was also reflected in the improved and more consistent accuracies for $\mathrm{T}$ staging ranging between 71 and $91 \%$ (19-26). With an endorectal MRI, however, the mesorectal fascia and surrounding pelvic structures are difficult to visualize due to the sudden signal drop off at a short distance from the coil (27), so endorectal MRI is less accurate for the evaluation of advanced rectal tumors.

With the introduction of dedicated coils, phased array coils, improvement in MRI performance was expected (28-32). The advantages of a high spatial resolution with a large field of view make phased array MRI suitable for staging of both superficial and advanced rectal tumors. This is shown in figures 2-5, which illustrate the phased array MR images of different stages of rectal cancer.

Nevertheless the first MRI studies that used the multiple surface coil technique reported an overall accuracy for $\mathrm{T}$ staging of only $55-65 \%$ and obviously showed no benefit as compared to the body coil MRI or even to CT $(33,34)$. The low performance of MRI in these studies could have been attributed to the low spatial resolution that was used with the early phased array techniques. But even when a higher spatial resolution had been applied with newer generation phased array coil MRI techniques, the accuracy for $\mathrm{T}$ staging was not as consistent and high as anticipated with figures varying between 65 and $86 \%$ and considerable inter-observer variability $(8,32,35-36)$. 


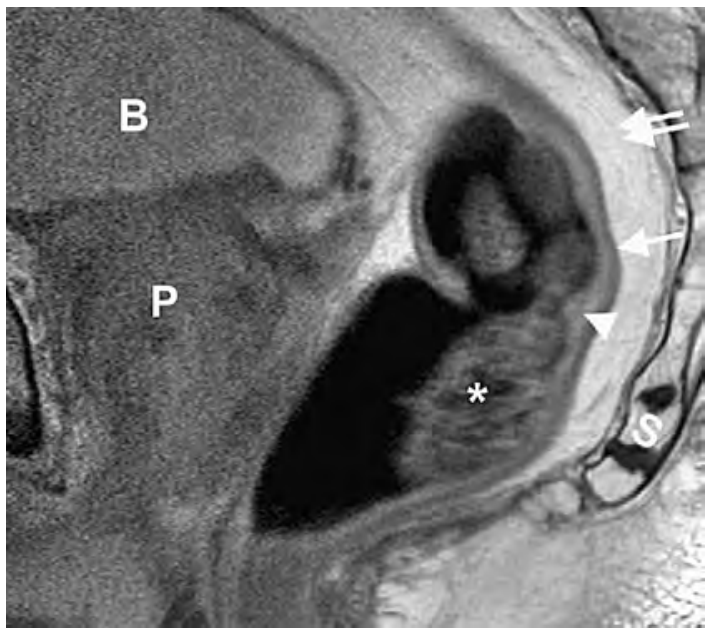

Fig. 2. MRI of T1 rectal cancer. Sagittal contrast-enhanced TIW TSE MR image showing the different rectal wall layers important for T staging. The rectal tumor (asterisk) is slightly hypointense to the hyper-intense submucosal layer (arrowhead) and slightly hyper-intense to the hypointense muscular rectal wall (arrow). There is tumor invasion in the submucosal layer but no invasion in the muscularis propria, stage $\mathrm{T} 1$ tumor. $\mathrm{B}=$ Bladder; $\mathrm{P}=$ prostate; $\mathrm{S}=$ sacrum.

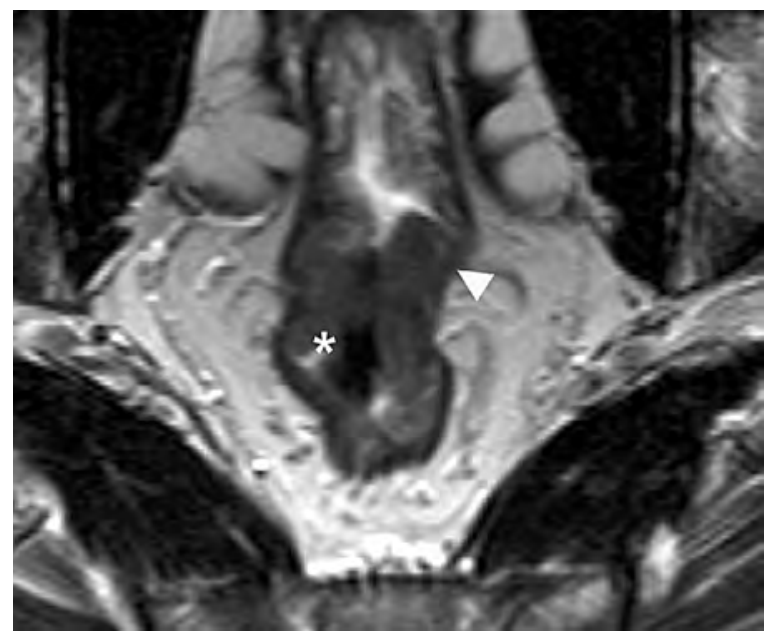

Fig. 3. MRI of $T 2$ rectal cancer Axial T2W TSE MR image shows a rectal tumor (asterisk), slightly hyper-intense to the muscular rectal wall (arrowhead). The tumor is limited to the rectal wall, there is no penetration into the perirectal fat, stage $\mathrm{T} 2$ rectal cancer. 


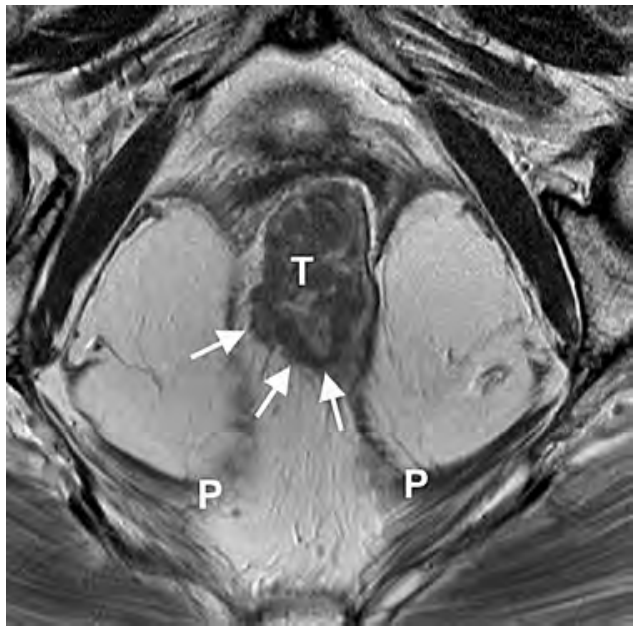

Fig. 4. $M R I$ of $T 3$ rectal cancer. Axial contrast-enhanced $T 1 W$ TSE MR image depicting a rectal tumor ( $\mathrm{T}$ ) with tumor penetration through the rectal wall into the mesorectal fat tissue (arrows). In contrast to the tumor in figure 9 , which shows a spiculated growth pattern, this tumor shows a more nodular growth pattern into the mesorectal fat, almost $100 \%$ predictive of tumor penetration through the rectal wall. $\mathrm{P}=$ Pelvic floor muscles.

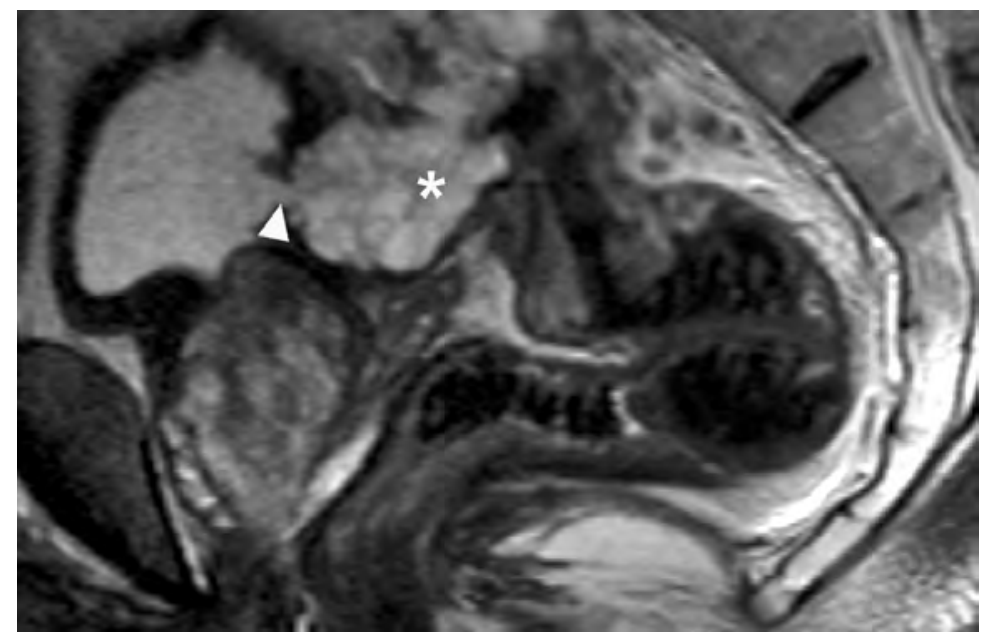

Fig. 5. MRI of T4 rectal cancer. Sagittal T2W TSE MR image of a hyper-intense rectal tumor (asterisk), invading and disrupting the dorsal bladder wall (arrowhead). This is the typical MR appearance of a mucinous adenocarcinoma.

\section{MRI techniques and circumferential resection margins}

So far there have been 4 reports in literature on the MRI evaluation of the mesorectal fascia and circumferential resection margins. In one study the mesorectal fascia was visualized with a high-resolution phased array MRI technique, and although the authors concluded that the depth of tumor 
extension could he predicted with high accuracy, the more relevant distance between tumor and fascia was not studied (3I). With a postoperative MRI of 26 resected rectal tumor specimens, Blomqvist et al. (37) were able to predict tumor involvement of the circumferential resection margin with high accuracy. The largest study to date on the MRI evaluation of circumferential resection margins in patients with rectal cancer was published by our team in the Lancet early 2001 (8). Seventy-six patients underwent a preoperative phased array $\mathrm{MRI}$ and the images were evaluated by 2 observers. The accuracy for T staging was $83 \%$ for observer I but only $67 \%$ for the less experienced observer 2 . For 12 T4 tumors involving the mesorectal fascia, both observers correctly predicted this in all 12 patients. In 29 patients who had a wide circumferential margin (>10 mm), observer I correctly predicted the margin in 28 and the less experienced observer 2 in 27 patients. For margins between 1 and $10 \mathrm{~mm}$, a linear regression curve constructed for both observers showed that the crucial distance of at least $2 \mathrm{~mm}$ can be predicted with $97 \%$ confidence when the distance on MRI is at least $6 \mathrm{~mm}$. An important finding was the high agreement of the resection margin measurements both between (intra-class correlation coefficients 0.99 and 0.91 ) and within the observers (intra-class correlation coefficient 0.93 ) in contrast to the only moderate intra- and inter-observer agreement for the T-stage determination (kappa 0.53). These results were confirmed in a study by Bissett et al. (7) on the MR determination of the circumferential resection margins in 43 patients. The authors reported a $95 \%$ accuracy on the MR prediction of tumor penetration through the mesorectal resection plane.

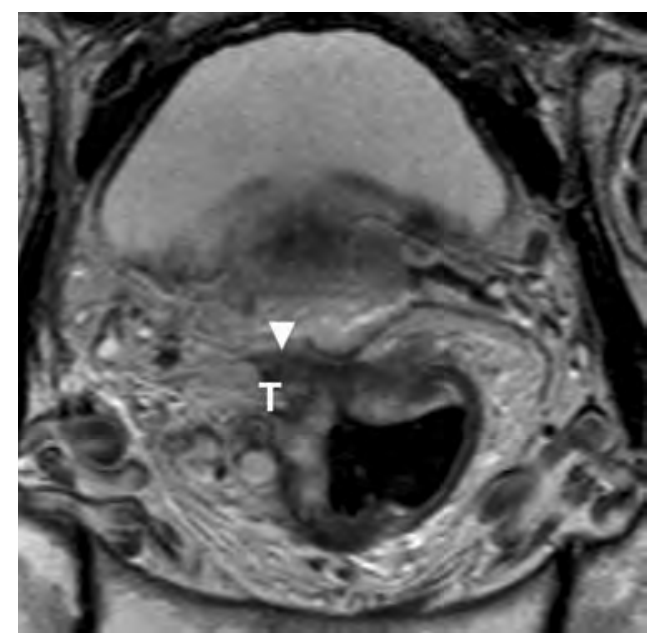

Fig. 6. $M R I$ of rectal cancer with invasion of the mesorectal fascia. Axial T2W TSE MR image shows a rectal tumor $(T)$ penetrating the rectal wall. There is no fat plane visible between the tumor and the thickened mesorectal fascia (arrowhead), suggesting invasion of this structure. This is important preoperative information because a wider excision than a total mesodermal excision is needed in this patient to obtain a free resection margin. 


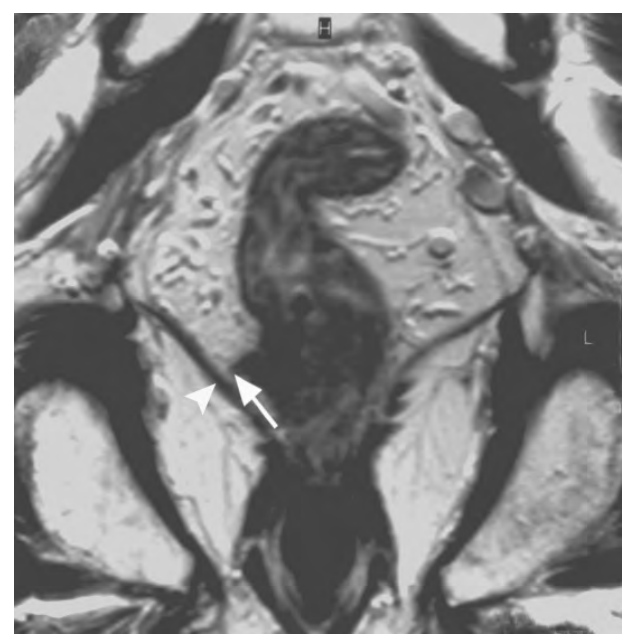

Fig. 7. MRI of rectal cancer with a close resection margin. Coronal T2W TSE MR image shows a distal rectal tumor in the right lateral wall (arrow), which has penetrated the rectal wall and extended close to the pelvic floor muscles (arrowhead). The measured distance between the tumor and mesorectal Fascia was $2 \mathrm{~mm}$ on MRI and $3 \mathrm{~mm}$ at histology MRI is very accurate in predicting the circumferential resection margin at total mesodermal excision.

This indicates that phased array MRI is very reliable for the prediction of the circumferential resection margin. The MR evaluation of the resection margin is more consistent and less affected by the skill of the readers than the MR evaluation of the T stage. Some of the findings of our study are illustrated in figures 6 and 7.

\section{MRI of rectal cancer: a word of caution}

Most staging failures with MRI occur in the differentiation of $\mathrm{T} 2$ and borderline T3 lesions with overstaging as the main cause of errors. Overstaging is often caused by desmoplastic reactions $(8,26,38)$ and it is difficult to distinguish between spiculation in the perirectal fat caused by fibrosis only (stage pT2) and spiculation caused by fibrosis that contains tumor cells (stage pT3) on MRI (8). Figures 8 and 9 illustrate this pitfall best. Both figures show similar MRI pictures of two patients with a rectal tumor and tumoral stranding into the mesorectal fat. The patient in figure 8 had a T2 rectal cancer, while the patient in figure 9 had a T3 rectal cancer. Differentiation between T2 and T3 tumors is difficult in the case of a spiculated growth pattern. A spiculated pattern is usually caused by a desmoplastic reaction around the tumor, but MRI cannot accurately distinguish between fibrosis with or without viable tumor cells. A nodular growth pattern, as shown in figure 4, however, is more predictive of tumor penetration through the rectal wall. 


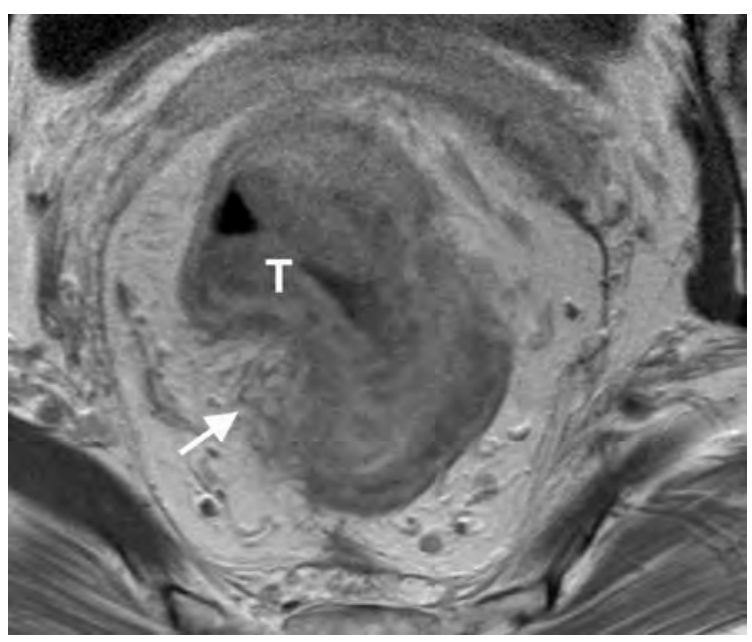

Fig. 8. $M R$ difficulties is distinguishing between fibrosis with and without viable tumor cells. Axial contrast-enhanced TI W MR image shows a rectal tumor (T) with speculated infiltration (arrow) in the mesorectal fat, suggesting a T3 tumor. The spiculations consist of desmoplastic reaction only, there were no tumor cells, histologically stage T2 tumor. MRI overstaged the tumor.

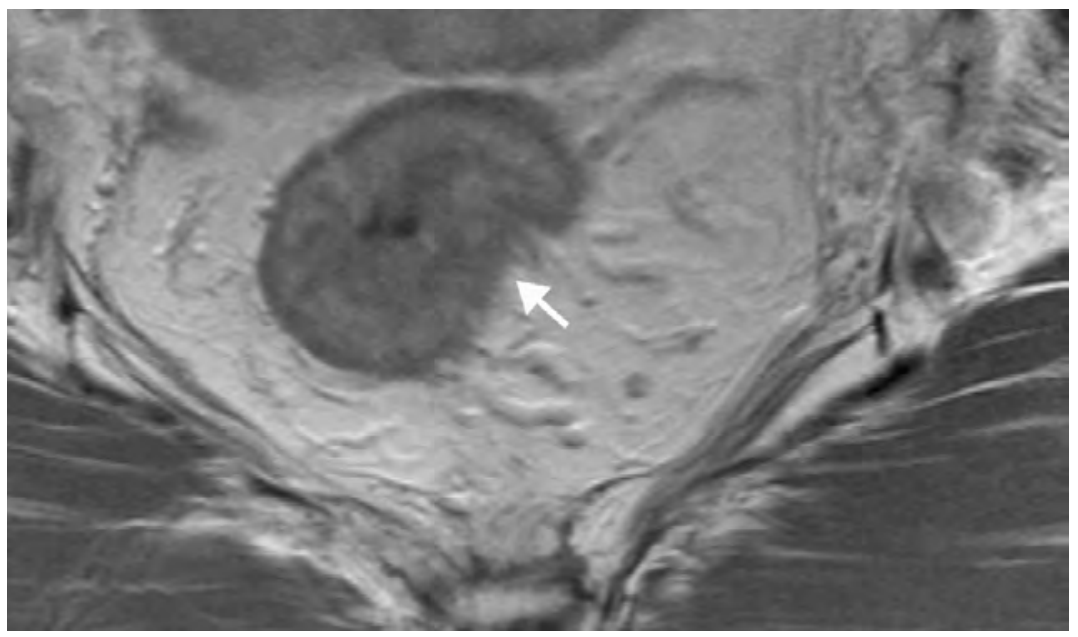

Fig. 9. MR difficulties in distinguishing between fibrosis with and without viable tumor cells. Axial contrast-enhanced TI W MR image of a rectal tumor shows a similar pattern of spiculations in the mesorectal fat as in figure 8 (arrow). However, in this patient these spiculations consisted of fibrosis with viable tumor cells, histologically stage T3 tumor. Differentiation between T2 and T3 tumors is difficult in case of a spiculated growth pattern. A spiculated pattern is usually caused by a desmoplastic reaction around the tumor, but MRI cannot accurately distinguish between fibrosis with or without viable tumor cells. 
Interpretation problems also occur on MRI of irradiated rectal cancer. Radiotherapy can cause tumor shrinkage, necrosis and fibrosis, as shown in figure 10. MRI can be useful to evaluate tumor response after radiotherapy, but one should be aware of some pitfalls (9). Figures 11 and 12 illustrate some of these pitfalls. A hypo-intense mass after radiotherapy generally represents fibrosis, but in some cases can contain viable tumor cells (39). Again MRI cannot reliably distinguish between fibrosis with or without viable tumor cells.

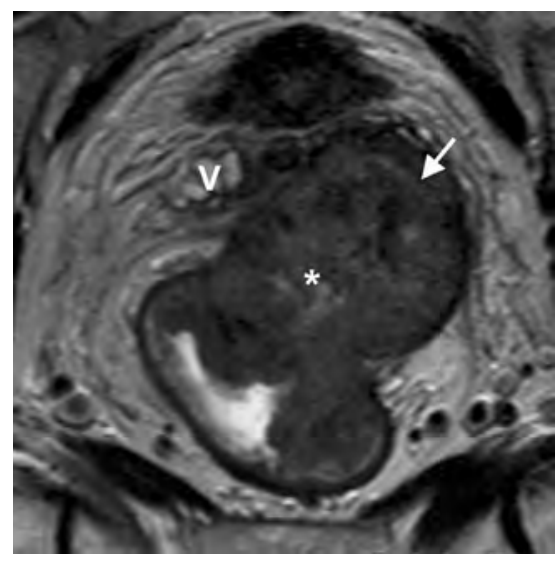

$10 a$

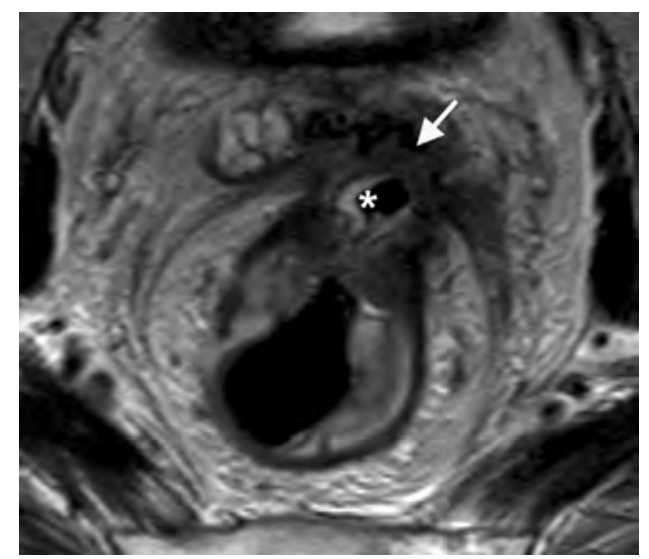

10b

Fig. 10a-b. MRI of rectal cancer before and after radiotherapy. (a) Axial T2W TSE MR image of rectal cancer before radiotherapy shows a bulky anterior located tumor (asterisk), which has penetrated the mesorectal fat and invaded the left seminal vesicle (arrow). $V=$ Normal seminal vesicle. (b) Axial T2W TSE MR image of the same patient after a long course of radiation therapy. There is a reduction in tumor size and overall the tumor has become more hypo-intense, suggestive of post-radiation fibrosis (arrow). The central part of the tumor has become hyper-intense or necrotic and there is also a central crater (asterisk).

When MRI shows these changes around the resection margin they may cause interpretation difficulties in predicting whether the resection margin will be free. In order to minimize these interpretation problems, one should make a baseline MRI before radiotherapy. Surgeons often dissect the whole area of fibrosis assuming that fibrosis on post-radiation MRI indicates a former tumor location. 

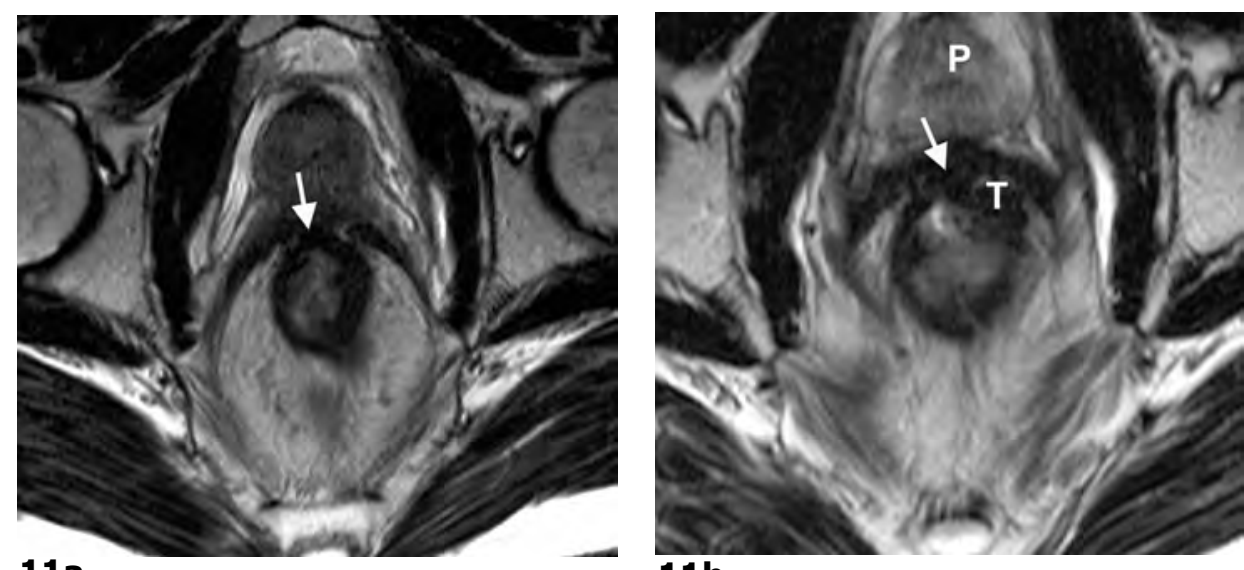

$11 \mathrm{a}$

$11 \mathbf{b}$

Fig. 11a-b. MRI of rectal cancer before and after radiotherapy. (a) Axial T2W TSE MR image of rectal cancer before radiotherapy shows an anteriorly located tumor $(T)$, slightly hyper-intense to the muscolaris propria, invading the pelvic floor muscles (arrow). $\mathrm{P}=$ Prostate. (b) Axial T2W MR image of the same patient after a long course of radiotherapy shows a reduction in the tumor size (arrow) and signal intensity, suggesting post-radiation fibrosis. In the resection specimen, no viable tumor cells were detected. The residual hypo-intense mass was based on fibrosis only.

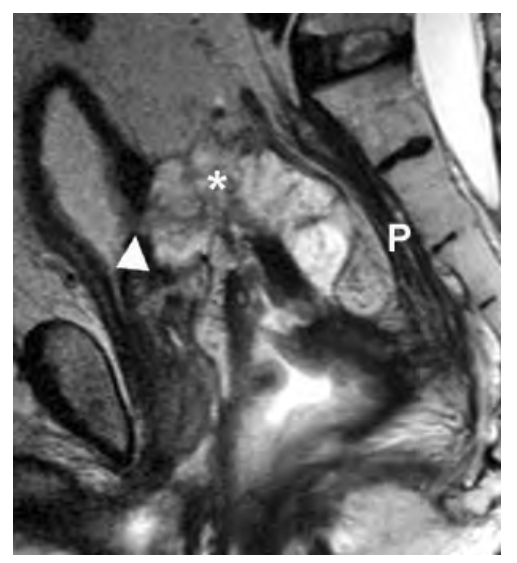

Fig. 12. MRI of rectal cancer after radiotherapy. Sagittal T2W TSE MR image shows a hyperintense tumor (asterisk) invading the dorsal bladder wall (arrow-head), suggestive of a mucinous adenocarcinoma. The presacral fascia is thickened $(\mathrm{P})$ and shows a very hypo-intense aspect after irradiation, resembling the radiation fibrosis in figure $11 \mathrm{~b}$. However, this presacral fascia contained viable tumor cells. MRI cannot reliably differentiate between fibrosis with or without tumor cells. 


\section{Conclusion}

MRI is a reliable imaging modality for the preoperative determination of the lateral tumor-free resection margin and local tumor extent in patients with rectal cancer. This allows an accurate preoperative selection by MRI of those patients with advanced tumors and a high risk of local recurrence who will benefit from more extensive (neoadjuvant) treatment. Nevertheless some difficulties can occur in reading MR images, especially of patients with irradiated rectal cancer, that prevents accurate prediction of the resection margins. Most interpretation difficulties are caused by post-radiation fibrosis. When MRI is going to be used for clinical decision making one should be aware not only of the normal MRI appearances of rectal cancer but also of the pitfalls of MRI in irradiated rectal cancer. This essay has provided an overview of significant MR findings in rectal cancer and discussed some of the pitfalls in interpreting MRI of irradiated rectal cancer.

\section{References}

1. Minsky B. Adjuvant radiation therapy for rectal cancer: Is there finally an answer? Lancet 2001;358: 1285-1286.

2. Colorectal Cancer Collaborative Group. Adjuvant radiotherapy for rectal cancer: A systematic overview of 8,507 patients from 22 randomised trials. Lancet 2001;358:12911304.

3. Swedish Rectal Cancer Trial: Improved survival with preoperative radiotherapy in resectable rectal cancer. N Engl J Med 1997; 336:980-987. Published erratum: N Engl J Med 1997; 336:1539.

4. Kapiteijn E, Marijnen C, Nagtegaal I, et al. Preoperative radiotherapy combined with total mesorectal excision for resectable rectal cancer. N Engl J Med 2001;345:638-646.

5. Heald R, Ryall R. Recurrence and survival after total mesorectal excision for rectal cancer. Lancet 1986; i: 1479-1482.

6. Chen $E$, Mohiuddin M. Brodovsky $H$, et al. Downstaging of advanced rectal cancer following combined preoperative chemotherapy and high dose radiation. Int J Radiat Oncol Biol Phys 1994;30:169-175.

7. Bissett I, Fernando C, Hough D, et al. Identification of the fascia propria by magnetic resonance imaging and its relevance to preoperative assessment of rectal cancer. Dis Colon Rectum 2001;44:259-265.

8. Beets-Tan R, Beets G, Vliegen R, et al. Accuracy of magnetic resonance imaging in prediction of tumour-free resection margin in rectal cancer surgery. Lancet 2001;357:497504.

9. Beets-Tan R, Beets G, Borstlap A, et al. Preoperative assessment of local tumor extent in advanced rectal cancer: CT or high-resolution MRI? Abdom I maging 2000;25:533-541.

10. Blomqvist L, Holm T, Nyren S, et al. MR Imaging and computed tomography in patients with rectal tumours clinically judged as locally advanced. Clin Radiol 2002;57:211-218.

11. Butch R, Stark D, Wittenberg J, et al. Staging rectal cancer by MR and CT. AJR Am J Roentgenol 1986;146:1155-1560.

12. Hodgman C, MacCarty R, Wolff B, et al. Preoperative staging of rectal carcinoma by computed tomography and $0.15 \mathrm{~T}$ magnetic resonance imaging. Preliminary report. Dis Colon Rectum 1986;29:446-450. 
13. Guinet C, Buy J, Ghossain M, et al. Comparison of magnetic resonance imaging and computed tomography in the preoperative staging of rectal cancer. Arch Surg 1990; 125:385-388.

14. Okizuka H, Sugimura K, Ishida T. Preoperative local staging of rectal carcinoma with MR imaging and a rectal balloon. J Magn Reson Imaging 1993;3:329-335.

15. Cova M, Frezza F, Pozzi-Mucelli R, et al. Computed tomography and magnetic resonance in the preoperative staging of the spread of rectal cancer. A correlation with the anatomicopathological aspects (in Italian). Radiol Med (Torino) 1994;87:82-89.

16. Zerhouni E, Rutter $\mathrm{C}$, Hamilton $\mathrm{S}$, et al. CT and MR imaging in the staging of colorectal carcinoma: Report of the Radiology Diagnostic Oncology Group II. Radiology 1996; 200:443-451.

17. Starck M, Bohe M, Fork $F$, et al. Endoluminal ultrasound and low-field magnetic resonance imaging are superior to clinical examination in the preoperative staging of rectal cancer. Eur J Surg 1995; 161:841-845.

18. Vogl T, Pegios W, Hunerbein M, et al. Use and applications of MRI techniques in the diagnosis and staging of rectal lesions. Recent Results Cancer Res 1998;146:35-47.

19. Gualdi G, Casciani E, Guadalaxara A, et al. Local staging of rectal cancer with transrectal ultrasound and endorectal magnetic resonance imaging: Comparison with histologic findings. Dis Colon Rectum 2000;43:338-345.

20. Maldjian C, Smith R, Kilger A, et al. Endorectal surface coil MR imaging as a staging technique for rectal carcinoma: A comparison study to rectal endosonography. Abdom Imaging 2000;25:75-80.

21. Chan T, Kressel H, Milestone B, et al. Rectal carcinoma: Staging at MR imaging with endorectal surface coil. Work in progress. Radiology 1991;181:461-467.

22. Schnall M, Furth E, Rosato E, et al. Rectal tumor stage: Correlation of endorectal MR imaging and pathologic findings. Radiology 1994;190:709-714.

23. Indinnimeo M, Grasso R, Cicchini $C$, et al. Endorectal magnetic resonance imaging in the preoperative staging of rectal tumors. Int Surg 1996;81:419-422.

24. Zagoria R, Schlarb C, Ott D, et al. Assessment of rectal tumor infiltration utilizing endorectal MR imaging and comparison with endoscopic rectal sonography. J Surg Oncol 1997;64:312-317.

25. Pegios $W$, Vogl J, Mack $M$, et al. MRI diagnosis and staging of rectal carcinoma. Abdom Imaging 1996;21:211-218.

26. Vogl T, Pegios W, Mack M, et al. Accuracy of staging rectal tumors with contrastenhanced transrectal MR imaging. AJ R Am J Roentgenol 1997;168:1427-1434.

27. deSouza N, Hall A, Puni R, et al. High resolution magnetic resonance imaging of the anal sphincter using a dedicated endoanal coil. Comparison of magnetic resonance imaging with surgical findings. Dis Colon Rectum 1996;39:926-934.

28. Beets-Tan R, Beets G, van der Hoop A, et al. High-resolution magnetic resonance imaging of the anorectal region without an endocoil. Abdom Imaging 1999;24:576-581; discussion 582-584.

29. Beets-Tan R, Morren G, Beets G, et al. Measurement of anal sphincter muscles: Endoanal US, endoanal MR imaging, or phased-array MR imaging? A study with healthy volunteers. Radiology 2001;220:81-89.

30. Beets-Tan R, Beets G, van der Hoop A, et al. Preoperative MR imaging of anal fistulas: Does it really help the surgeon? Radiology 2001;218:75-84.

31. Brown G, Richards C, Newcombe R, et al. Rectal carcinoma: Thin-section MR imaging for staging in 28 patients. Radiology 1999;211:215-222.

32. Blomqvist L, Machado M, Rubio $C$, et al. Rectal tumour staging: MR imaging using pelvic phased-array and endorectal coils vs endoscopic ultrasonography. Eur Radiol 2000; 10:653-660. 
33. Hadfield M, Nicholson A, MacDonald A, et al. Preoperative staging of rectal carcinoma by magnetic resonance imaging with a pelvic phased-array coll. Br J Surg 1997;84:529-531.

34. Lange de $E$, Fechner $R$, Wanebo $H$. Suspected recurrent rectosigmoid carcinoma after abdominoperineal resection: $M R$ imaging and histopathologic findings. Radiology 1989; 170: 323-328.

35. Blomqvist L, Holm T, Rubio C, et al. Rectal tumours -MR imaging with endorectal and/or phased-array coils, and histopathological staging on giant sections. A comparative study. Acta Radiol 1997; 38:437-444.

36. Gagliardi G, Bayar S, Smith R, et al. Preoperative staging of rectal cancer using magnetic resonance imaging with external phase-arrayed coils. Arch Surg 2002;137:447-451.

37. Blomqvist L, Rubio C, Holm T, et al. Rectal adenocarcinoma: Assessment of tumour involvement of the lateral resection margin by MRI of resected specimen. $\mathrm{Br} J$ Radiol 1999; 72:18-23.

38. Meyenberger $\mathrm{C}$, Huch Boni R, Bertschinger $\mathrm{P}$, et al. Endoscopic ultrasound and endorectal magnetic resonance imaging: A prospective, comparative study for preoperative staging and follow-up of rectal cancer. Endoscopy 1995;27:469-479.

39. Kahn H, Alexander A. Rakinic J, et al. Preoperative staging of irradiated rectal cancers using digital rectal examination, computed tomography, endorectal ultrasound, and magnetic resonance imaging does not accurately predict TO, NO pathology. Dis Colon Rectum 1997; 40:140-144. 



\section{Rectal cancer: MR Imaging in local}

staging-Is gadolinium-based contrast material helpful?

RF Vliegen, GL Beets, MF von Meyenfeldt, AG Kessels, EE Lemaire, J M van Engelshoven, RG Beets-Tan.

Radiology 2005; 234:179-188. 


\section{Abstract}

Purpose: To determine retrospectively whether addition of gadoliniumenhanced T1-weighted magnetic resonance (MR) sequence to T2-weighted turbo spin-echo (SE) MR imaging is valuable for preoperative assessment of $T$ stage and circumferential resection margin in patients with primary rectal cancer.

Materials and Methods: Eighty-three patients with operable primary rectal cancer underwent preoperative MR imaging. Retrospectively, two observers independently scored T2-weighted turbo SE MR images and, in a second reading, T2-weighted images combined with gadolinium-enhanced T1-weighted turbo SE MR images for tumor penetration through rectal wall and tumor extension into mesorectal fascia. A confidence level scoring system was used, and receiver operating characteristic (ROC) curves were generated. Histologic findings were standard of reference. Difference in performance of T2weighted and combined T2-weighted plus gadolinium-enhanced T1-weighted sequences was analyzed by comparing corresponding areas under ROC curves $\left(A_{z}\right)$ for each observer. Interobserver agreement was calculated by using linear weighted $\mathrm{k}$ statistics.

Results: Addition of contrast enhanced T1-weighted to T2-weighted images did not significantly improve the diagnostic accuracy for predicting tumor penetration through the rectal wall ( $A z$ of $\mathrm{T} 2$-weighted vs $\mathrm{T} 2$-weighted plus contrast enhanced T1-weighted images for obs $1,0.740$ vs 0.764 ; obs. $2,0.856$ vs 0.768 ) and tumor extension into the mesorectal fascia (Az for observer. $1,0.962$ vs 0.902 ; observer $2,0.902$ vs 0.911 ). Diagnostic performance $(\mathrm{Az})$ of $\mathrm{MRI}$ and interobserver agreement were high for prediction of tumor extension intoof the mesorectal fascia $(\kappa=0.61,0.74)$ but only moderate for penetration through the rectal wall $(\kappa=0.47,0.45)$.

Conclusion: Gadolinium enhanced MR sequences did not improve diagnostic accuracy for assessment of tumor penetration through the rectal wall and tumor extension into mesorectal fascia. 


\section{Introduction}

In recent years, surgeons and radiation therapists have directed their efforts at reducing the local recurrence rate after resection of primary rectal cancer. Incomplete removal of the lateral spread of tumor is generally recognized as the major cause of local recurrence rate, varying from $3 \%$ up to $32 \%(1-4)$. With a standardized surgical technique-total mesorectal excision-a distinct anatomic compartment called the mesorectum, containing the rectum and the mesorectal fat, is removed by means of sharp dissection along the mesorectal fascia. This results in a substantial reduction of the high local recurrence rate to less than $10 \%$ (5) Postoperative and especially preoperative radiation therapy have also shown a substantial reduction of local recurrence rates (6-7). A large randomized trial (8) showed that even with optimal surgical technique, a preoperative short course of radiation therapy even further reduces the local recurrence rate to less than $5 \%$.

Although postoperative radiation therapy is still practiced widely in the United States, there is an increasing interest in applying it preoperatively on the basis of data that suggest an improved efficacy. At the same time, the idea of differentiated treatment is gaining ground: On the basis of the risk for local recurrence, patients could be treated with surgery only for cases of superficial tumors, with a short course of preoperative radiation therapy for intermediate cases, or with a long course of preoperative radiochemotherapy for locally advanced cases. This can be achieved only with good preoperative imaging of rectal cancer.

Magnetic resonance (MR) imaging is the most promising technique for the local staging of rectal cancer (9). The identification of tumors close to or invading the mesorectal fascia has become increasingly important, maybe even more important than the classic $T$ stage determination (10).

High-spatial-resolution T2-weighted fast spin-echo (SE) MR sequences performed with a phased-array coil have been shown to produce a detailed depiction of the mesorectal fascia and allow accurate prediction of the circumferential resection margin (11-12). The role of contrast materialenhanced MR sequences is not clear. There are few publications on the use of contrast materials in MR imaging of rectal cancer (13-16). Most of these studies involve the comparison of contrast-enhanced MR imaging with nonenhanced MR imaging for the prediction of $T$ stage, but because of the different techniques applied, these studies are difficult to compare. To our knowledge, there is no report on the benefit of contrast-enhanced MR sequences for the prediction of tumor invasion into the mesorectal fascia.

The purpose of this study was to determine retrospectively whether the addition of gadolinium-enhanced T1-weighted MR sequences to T2-weighted turbo SE MR sequences is valuable for preoperative assessment of $T$ stage and circumferential resection margin in patients with primary rectal cancer. 


\section{Materials and Methods}

Institutional approval was obtained for this retrospective study. Between July 1997 and April 2001, a total of 83 consecutive patients with primary operable rectal cancer who underwent surgery following standard preoperative MR imaging that included gadolinium-enhanced sequences were included retrospectively. There were 61 male patients with a mean age of 64 years (age range, $15-85$ years) and 22 female patients with a mean age of 66 years (age range, 36-86 years).

\section{Imaging techniques}

MR imaging was performed with a 1.5-T system (Gyroscan, Powertrack 6000 , NT release 6.2 .1 with $23.0 \mathrm{mT} / \mathrm{m}$, rise time of $0.2 \mathrm{msec}$, and slew rate $105 \mathrm{~T} / \mathrm{m} / \mathrm{sec}$; Philips Medical Systems, Best, the Netherlands). The patients were positioned supine with feet in the first position with the pelvis centered on the proximal end of a quadrature phased-array spine coil (Synergy spine coil; Philips Medical Systems).

The following MR imaging sequences were applied: a precontrast T1weighted two-dimensional turbo (fast) SE sequence (repetition time msec/echo time msec, 656/10; echo train length of five, 8- $\mathrm{mm}$ section thickness, $0.8-\mathrm{mm}$ gap, four signals acquired, $166 \times 256$ matrix, $25-\mathrm{cm}$ field of view, and acquisition time of 1.4 minutes), a gadolinium-enhanced $(0.2 \mathrm{~mL}$ per kilogram of body weight, Magnevist; Schering, Berlin, Germany) T1-weighted twodimensional turbo (fast) SE sequence $(612 / 15$, echo train length of five, 3-mm section thickness, 0.3-mm gap, eight signals acquired, $383 \times 512$ matrix, $20-\mathrm{cm}$ field of view, $0.6-\mathrm{mm}^{3}$ voxel size, and acquisition time of 9.0 minutes), and a T2-weighted two-dimensional turbo (fast) SE sequence (3427/ 150, echo train length of $25,3-\mathrm{mm}$ section thickness, $0.3-\mathrm{mm}$ gap, eight signals acquired, $175 \times 256$ matrix, $20-\mathrm{cm}$ field of view, $2.64-\mathrm{mm}^{3}$ voxel size, and acquisition time of 6.5 minutes).

The T1-weighted precontrast MR sequence was performed in the transverse plane, and the first T2-weighted MR sequence was performed in the sagittal plane. These images were used to plan the T2-weighted sequences in the transverse and coronal planes. The transverse and coronal planes were angled exactly perpendicular to the long axis of the rectal tumor. Gadoliniumenhanced T1-weighted sequences were performed in the transverse and coronal planes.

Patients did not receive bowel preparation, air insufflation, or intravenous spasmolytic medication. The total imaging time was approximately 45 minutes. 


\section{Image evaluation}

The MR images were evaluated retrospectively by two radiologists, who were blinded to each other and to the histologic results. Observer 1 was a dedicated pelvic MR imaging radiologist (R.G.H.B.T.) who had more than 10 years of experience in reading pelvic MR images. Observer 2 was a general radiologist (R.F.A.V.) who had 5 years of experience in reading MR images.

Both observers scored the MR images independently for tumor penetration through the muscular rectal wall and tumor extension into the mesorectal fascia by using a confidence level scoring system. The following confidence levels were used: definitely absent, probably absent, possibly present, probably present, and definitely present.

During a first reading only, the T2-weighted fast SE MR sequences in three orientations (sagittal, coronal, and transverse) were evaluated. During a second reading after at least a 1-week interval and in a different order, both T2-weighted turbo SE images and gadolinium-enhanced T1-weighted images (transverse and coronal) were evaluated. When there were MR images available that were obtained before radiation therapy in the group of patients that received preoperative irradiation, these images were scored for the prediction of tumor penetration through the rectal wall and extension into the mesorectal fascia.

The confidence levels for the prediction of tumor penetration through the rectal wall on MR images were defined as follows: definitely absent, completely intact muscular rectal wall; probably absent, disruption of the muscular rectal wall without extension beyond the contour of the rectal wall; possibly present, subtle spiculations in the perirectal fat or a minimal bulging tumor margin beyond the contour of the rectal wall; probably present, spiculations in the perirectal fat or a bulging tumor margin beyond the contour of the rectal wall; and definitely present, nodular invasion of the perirectal fat or diffuse stranding from the tumor into the perirectal fat with complete disruption of the muscular rectal wall.

On MR images, the mesorectal fascia was defined as the fine linear structure enveloping the mesorectum that was hypointense on T2-weighted images and isointense to muscle on contrast-enhanced T1-weighted images. The confidence levels for the prediction of tumor (or nodal) extension into the mesorectal fascia were defined as follows: definitely absent, a wide fat plane between the tumor and the mesorectal fascia; probably absent, a thin fat plane between the tumor and the mesorectal fascia; possibly present, a thin fat plane between the tumor and the fascia with subtle spiculations from the tumor reaching the mesorectal fascia; probably present, clear spiculations from the tumor reaching the mesorectal fascia or a minimal fat plane between the tumor, tumor deposit, or a suspected lymph node and the mesorectal fascia; and definitely present, diffuse stranding from the tumor into the mesorectal fascia, a 
nodular tumor mass encroaching the mesorectal fascia (figure 1), or a tumor deposit or suspected lymph node in direct contact with the mesorectal fascia.

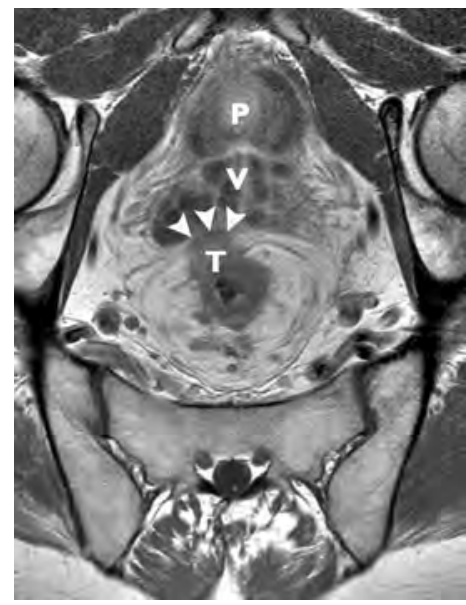

Fig. 1. Clear invasion of mesorectal fascia. Transverse gadolinium-enhanced T1-weighted MR image $(612 / 15)$ of rectal tumor $(T)$ with penetration (arrowheads) through rectal wall into mesorectal fat and fascia. There is no fat plane between anterior penetrating tumor and nodular thickened mesorectal fascia, which suggests invasion of this structure. This was confirmed at histologic examination. $P=$ prostate, $V=$ seminal vesicle.

On the combined T2-weighted plus gadolinium-enhanced T1-weighted MR images, observer 1 assessed whether there was a nodular advancing tumor margin present in the perirectal fat or an advancing tumor margin consisting of spiculations into the perirectal fat. This was done to define the predictive value of these patterns of invasion of the perirectal fat for the prediction of tumor penetration through the rectal wall. A nodular advancing tumor margin was defined as bulky tissue extending from the tumor into the perirectal fat. An advancing tumor margin consisting of spiculations was defined as fine strands of tissue extending from the tumor into the perirectal fat.

\section{Preoperative radiation therapy and surgery}

The standard surgical resection was a total mesorectal excision (5). Forty-two patients underwent sphincter-sparing total mesorectal excision, and six patients underwent non-sphincter-sparing abdominoperineal resection. In 29 patients, the surgical resection was extended to include surrounding pelvic structures or organs to obtain a free resection margin. Two patients underwent transanal resection because of a superficial rectal tumor. Four patients underwent explorative laparotomy with biopsy because of noncurable extensive disease. Twenty-seven patients received preoperative radiation therapy because of (a) suspected extensive rectal cancer, for which a long course of radiation therapy was administered in an attempt to downstage the tumor, or (b) participation in the Dutch randomized Total Mesorectal Excision trial, in which a short course of radiation therapy was administered ( $5 \times 5 \mathrm{~Gy}$ ) (8). Six of the 27 patients underwent MR imaging before radiation therapy only, 10 of 27 patients underwent MR imaging after radiation therapy only, and 11 of 27 patients underwent MR imaging both before and after radiation therapy. 


\section{Histologic examination}

Since the mid-1990s, the histologic evaluation of rectal cancer excision specimens has been standardized according to the protocol of Quirke et al (3). All evaluations are performed or supervised by dedicated pathologists with more than 10 years of experience in gastrointestinal pathology. The circumferential resection plane of the specimen was stained, and the specimen was opened anteriorly and fixed in formalin for 24 hours. The specimen was then sliced transversely at $0.5-\mathrm{cm}$ intervals. The extent of lateral tumor spread into the mesorectal fat was assessed macroscopically on each slice, and the shortest distance between the tumor or involved lymph node and the mesorectal circumferential resection plane was measured. When at macroscopy the circumferential resection margin was close to or involved with tumor tissue, the distance between the tumor and the circumferential stained resection plane was measured at microscopy. When adjacent organs were resected or when a resection was outside the plane of the mesorectal fascia, the relationship between the tumor and the mesorectal fascia was reconstructed. When a tumor response to radiation therapy was noted, the area of the obvious tumor necrosis and fibrosis was considered as the former tumor extent. In a few patients, the tumor appeared to be nonresectable at surgery. The distance of the tumor to the mesorectal fascia was reconstructed on the basis of intraoperative findings and biopsy results.

\section{Statistical analysis}

Tumor penetration through the muscular rectal wall and tumor extension into the mesorectal fascia at histologic examination were used as the standard of reference against which MR findings were compared (R.G.H.B.T., R.F.A.V.). A histologically measured distance of up to $1 \mathrm{~mm}$ between the tumor or a pathologic lymph node and the mesorectal fascia was considered to be tumor extension into the mesorectal fascia.

Receiver operating characteristic (ROC) curves were constructed (A.G.H.K., R.F.A.V.) with the confidence level scores $(0=$ absent, $1=$ probably absent, $2=$ possibly present, $3=$ probably present, and $4=$ present) at both readings ( $T 2$-weighted MR images and T2-weighted plus gadolinium-enhanced T1-weighted MR images) and for both observers for the detection of tumor penetration through the muscular rectal wall and for tumor extension into the mesorectal fascia.

The difference in performance between T2-weighted MR sequences only and the combined T2-weighted plus gadolinium-enhanced T1-weighted MR sequences was analyzed (A.G.H.K., R.F.A.V.) by means of comparison of the corresponding areas under the ROC curves $\left(A_{z}\right)$ for each observer (17). 
For the analysis of the influence of preoperative radiation therapy, age, and sex on the results, a subgroup analysis was performed (A.G.H.K., R.F.A.V.). The following subgroups were analyzed: patients with versus those without preoperative radiation therapy, male versus female patients, and age less than 60 years versus age more than 60 years. In each subgroup, ROC curves were constructed, and the difference in performance of T2-weighted and the combined T2-weighted plus gadolinium-enhanced T1-weighted sequences was evaluated by comparing $A_{z}$ values for the prediction of penetration through the rectal wall and tumor extension into the mesorectal fascia for each observer (17).

Findings that were missed on MR images at one or both readings for the whole group of patients and the subgroups were reviewed (R.G.H.B.T., R.F.A.V.) to find a specific cause.

For the calculation (A.G.H.K., R.F.A.V.) of the accuracy, sensitivity, specificity, positive predictive value (PPV), and negative predictive value (NPV) of MR imaging for the prediction of tumor extension into the mesorectal fascia and penetration through the rectal wall, the confidence level scores of each reading were dichotomized. The cutoff point was assigned between possibly present and probably present (between confidence levels of 0-2 and 3-4).

The accuracy, sensitivity, specificity, PPV, and NPV of a nodular advancing tumor margin into the perirectal fat and a tumor margin consisting of spiculations into the perirectal fat visualized on the combined T2-weighted plus gadolinium-enhanced T1-weighted MR images for the prediction of tumor penetration through the rectal wall were calculated (A.G.H.K., R.F.A.V.) for observer 1.

The interobserver agreement of the MR imaging evaluation was calculated A.G.H.K., R.F.A.V.) by using linear weighted $\mathrm{k}$ statistics (18). $\mathrm{K}$ values can range from 0 (no agreement) to 1.00 (perfect agreement) and can be interpreted as poor (0), slight $(0.01-0.20)$, fair $(0.21-0.40)$, moderate (0.41-0.60), substantial (0.61-0.80), and almost perfect (0.81-1.00) (19).

Statistical analysis was performed (A.G.H.K., R.F.A.V.) by using the software package

\section{Results}

\section{Tumor penetration of the rectal wall}

The accuracy, sensitivity, specificity, PPV, and NPV of T2-weighted images and combined T2-weighted plus gadolinium-enhanced T1-weighted MR images for the prediction of tumor penetration of the rectal wall for observers 1 and 2 are given in table 1 .

The $A_{z}$ values with $95 \%$ confidence intervals $(\mathrm{Cls})$ of T2-weighted MR images only and T2-weighted plus gadolinium-enhanced T1-weighted MR images were $0.74(95 \% \mathrm{Cl}: 0.61,0.87)$ and $0.76(95 \% \mathrm{Cl}: 0.63,0.90)$, 
respectively, for observer 1 and $0.86(95 \% \mathrm{Cl}: 0.75,0.96)$ and $0.77(95 \% \mathrm{Cl}$ : $0.63,0.91$ ), respectively, for observer 2 (table 1 , figure 2 ). There was no significant difference between the $A_{z}$ values of T2-weighted MR images only and T2-weighted plus contrast-enhanced T1-weighted MR images for both observers $(P=.71$ for observer 1 and $P=.17$ for observer 2$)$.

Table 1. Prediction of tumor penetration of the rectal wall and invasion of the mesorectal fascia.

\begin{tabular}{lllll}
\hline & \multicolumn{2}{c}{ Observer 1 } & \multicolumn{2}{c}{ Observer 2 } \\
\hline & T2-w & CE T1+ & CE T1+ \\
Parameters & T2-w & T2-w & T2-w \\
\hline Tumor penetration of rectal wall & & & \\
Az & 0.74 & 0.76 & 0.86 & 0.77 \\
$95 \% \mathrm{Cl}$ & $0.61-0.87$ & $0.63-0.90$ & $0.75-0.96$ & $0.63-0.91$ \\
Accuracy (\%) & $69(57 / 83)$ & $81(67 / 83)$ & $83(69 / 83)$ & $84(70 / 83)$ \\
Sensitivity (\%) & $78(49 / 63)$ & $87(55 / 63)$ & $91(57 / 63)$ & $98(62 / 63)$ \\
Specificity (\%) & $40(8 / 20)$ & $60(12 / 20)$ & $60(12 / 20)$ & $40(8 / 20)$ \\
PPV (\%) & $80(49 / 61)$ & $87(55 / 63)$ & $88(57 / 65)$ & $84(62 / 74)$ \\
NPV (\%) & $36(8 / 22)$ & $60(12 / 20)$ & $67(12 / 18)$ & $89(8 / 9)$ \\
& & & & \\
Invasion of the mesorectal fascia & 0.97 & 0.90 & & \\
Az & $0.93-1.01$ & $0.83-0.98$ & $0.83-0.98$ & $0.84-0.98$ \\
95\% Cl & $93(77 / 83)$ & $88(73 / 83)$ & $87(72 / 83)$ & $87(72 / 83)$ \\
Accuracy (\%) & $88(30 / 34)$ & $85(29 / 34)$ & $82(28 / 34)$ & $79(27 / 34)$ \\
Sensitivity (\%) & $96(47 / 49)$ & $90(44 / 49)$ & $90(44 / 49)$ & $92(45 / 49)$ \\
Specificity (\%) & $94(30 / 32)$ & $85(29 / 34)$ & $85(28 / 33)$ & $87(27 / 31)$ \\
PPV (\%) & $90(44 / 49)$ & $92(47 / 51)$ & $88(44 / 50)$ & $87(45 / 52)$ \\
NPV (\%) & & &
\end{tabular}

Note. - Number in parenthesis are raw data. CE T1+T2- $\mathrm{W}=$ contrast enhanced $\mathrm{T} 1$-weighted plus $\mathrm{T} 2$-weighted sequences, $\mathrm{T} 2-\mathrm{w}=\mathrm{T} 2$-weighted sequences only, $\mathrm{Az}=\mathrm{Area}$ under the $\mathrm{ROC}$ (Receiver Operating Curve), $95 \% \mathrm{Cl}=95 \%$ Confidence Interval. There was no statistical difference between $\mathrm{Az}$ values.

Subgroup analysis of patients with versus those without preoperative radiation therapy, male versus female patients, and age less than 60 years versus age more than 60 years showed that there was only a significant difference between the $A_{z}$ values of T2-weighted MR sequences and gadoliniumenhanced T1-weighted MR sequences in the group of patients with preoperative radiation therapy for observer 2 . T2-weighted sequences performed significantly better than the combined T2-weighted plus gadolinium-enhanced T1-weighted sequences in this subgroup of patients $(P<.05)$. The $A_{z}$ values with $95 \% \mathrm{Cls}$, accuracy, sensitivity, specificity, PPV, and NPV of T2-weighted images and combined T2-weighted plus gadolinium-enhanced T1-weighted images for the prediction of tumor penetration of the rectal wall in the group of patients with and those without preoperative radiation therapy for both observers are given in tables 2 and 3. 
Table 2. Subgroup analysis of patients without preoperative radiotherapy for the prediction of tumor penetration of the rectal wall and invasion of the mesorectal fascia.

\begin{tabular}{lllll}
\hline & \multicolumn{2}{c}{ Observer 1 } & \multicolumn{2}{c}{ Observer 2 } \\
\hline Parameters & T2-w & $\begin{array}{l}\text { CE T1+ } \\
\text { T2-w }\end{array}$ & T2-w & $\begin{array}{l}\text { CE T1+ } \\
\text { T2-w }\end{array}$ \\
\hline Tumor penetration of rectal wall & & & \\
$\mathrm{Az}$ & 0.70 & 0.80 & 0.83 & 0.83 \\
$95 \% \mathrm{Cl}$ & $0.55-0.85$ & $0.66-0.95$ & $0.70-0.96$ & $0.69-0.97$ \\
Accuracy (\%) & $70(39 / 56)$ & $73(41 / 56)$ & $79(44 / 56)$ & $84(47 / 56)$ \\
Sensitivity (\%) & $67(26 / 39)$ & $72(28 / 39)$ & $77(30 / 39)$ & $90(35 / 39)$ \\
Specificity (\%) & $77(13 / 17)$ & $76(13 / 17)$ & $82(14 / 17)$ & $71(12 / 17)$ \\
& & & & \\
Invasion of the mesorectal fascia & 0.98 & 0.90 & & \\
Az & $0.94-1.00$ & $0.79-1.00$ & $0.68-0.99$ & $0.75-1.00$ \\
$95 \%$ Cl & $91(51 / 56)$ & $84(47 / 56)$ & $86(48 / 56)$ & $88(49 / 56)$ \\
Accuracy & $85(11 / 13)$ & $85(11 / 13)$ & $77(10 / 13)$ & $85(11 / 13)$ \\
Sensitivity & $93(40 / 43)$ & $84(36 / 43)$ & $88(38 / 43)$ & $84(36 / 43)$ \\
Specificity & & & &
\end{tabular}

Note. - Number in parenthesis are raw data. CE $T 1+T 2-W=$ contrast enhanced $T 1$-weighted plus T2-weighted sequences, T2- $\mathrm{w}=\mathrm{T} 2$-weighted sequences only, Az=Area under the ROC (Receiver Operating Curve), $95 \% \mathrm{Cl}=95 \%$ Confidence Interval. There was no statistical difference between Az values.

Table 3. Subgroup analysis of patients with preoperative radiotherapy for the prediction of tumor penetration of the rectal wall and invasion of the mesorectal fascia.

\begin{tabular}{|c|c|c|c|c|}
\hline \multirow[b]{3}{*}{ Parameters } & \multicolumn{2}{|c|}{ Observer 1} & \multicolumn{2}{|c|}{ Observer 2} \\
\hline & & CE T1+ & & CE T1+ \\
\hline & T2-w & T2-w & T2-w & T2-w \\
\hline \multicolumn{5}{|c|}{ Tumor penetration of rectal wall } \\
\hline $\mathrm{Az}$ & $0.78^{*}$ & $0.48 *$ & $0.92 * *$ & $0.46^{* *}$ \\
\hline $95 \% \mathrm{Cl}$ & $0.45-1.00$ & $0.14-0.82$ & $0.82-1.00$ & 0.13-079 \\
\hline Accuracy (\%) & $89(24 / 27)$ & $85(23 / 27)$ & $85(23 / 27)$ & $81(22 / 27)$ \\
\hline Sensitivity (\%) & $92(22 / 24)$ & $96(23 / 24)$ & $92(22 / 24)$ & $92(22 / 24)$ \\
\hline Specificity (\%) & $67(2 / 3)$ & $0(0 / 3)$ & $33(1 / 3)$ & $0 \quad(0 / 3)$ \\
\hline
\end{tabular}

Invasion of the mesorectal fascia

$\begin{array}{lllll}\text { Az } & 0.96 * & 0.87 * & 0.95 * & 0.90 * \\ \text { C.I. 95\% } & 0.88-1.00 & 0.67-1.00 & 0.86-1.00 & 0.78-1.00 \\ \text { Accuracy (\%) } & 93(25 / 27) & 85(23 / 27) & 89(24 / 27) & 78(21 / 27) \\ \text { Sensitivity (\%) } & 95(20 / 21) & 86(18 / 21) & 90(19 / 21) & 76(16 / 21) \\ \text { Specificity (\%) } & 83(5 / 6) & 83(5 / 6) & 83(5 / 6) & 83(5 / 6)\end{array}$

Note. - Number in parenthesis are raw data. CE T1+T2- $w=$ contrast enhanced T1-weighted plus T2-weighted sequences, T2-w=T2-weighted sequences only, Az=Area under the ROC (Receiver Operating Curve), $95 \% \mathrm{Cl}=95 \%$ Confidence Interval.

* No statistical difference between Az values.

** Significant difference $(p<0.05)$ between Az values 


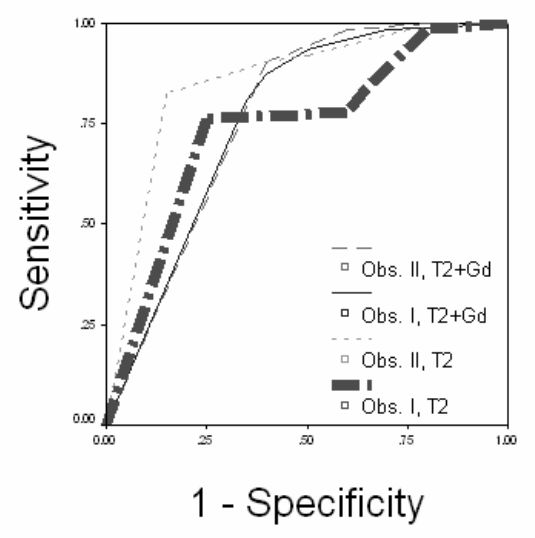

Fig. 2. Diagnostic performance represented by ROC curves of T2-weighted MR sequences (T2) and T2-weighted sequences plus contrast-enhanced T1-weighted MR sequences $(T 2+G d)$ for prediction of tumor penetration through rectal wall for observers 1 (Obs. I) and 2 (Obs. II). There was no significant difference $(P>.05)$ between $A_{z}$ values of T2-weighted sequences and combined T2-weighted sequences plus contrast-enhanced T1-weighted sequences. Diagnostic performance of MR imaging for prediction of rectal wall penetration for both observers appeared to be moderate: $A_{z}=0.74-0.86$.

Sixty-three tumors breached the rectal wall, and 20 tumors were confined to the rectal wall. On T2-weighted MR images only, observers 1 and 2 overstaged 12 and eight tumors and understaged 14 and six tumors, respectively. After the addition of gadolinium-enhanced T1-weighted images, observers 1 and 2 overstaged eight and 12 tumors and understaged eight tumors and one tumor, respectively.

Most cases of inaccurate staging were caused by difficulties of the interpretation of spiculations into the perirectal fat (figures $3 a, 3 b$ ). This occurred in patients without preoperative radiation therapy, as well as in a few irradiated patients with superficial tumors. These spiculations consisted histologically of a desmoplastic tumor reaction or radiation-induced fibrosis with or without tumor nests that could not be discriminated on T2-weighted MR images or on gadolinium-enhanced T1-weighted MR images. No specific cause could be found for the better performance of T2-weighted sequences in the group of patients with preoperative radiation therapy for observer 2 .

The accuracy, sensitivity, specificity, PPV, and NPV of a nodular advancing tumor margin into the perirectal fat (figure $3 \mathrm{c}$ ) or a tumor margin consisting of spiculations (figure $3 \mathrm{a}, 3 \mathrm{~b}$ ) for the prediction of tumor penetration through the rectal wall, as assessed by observer 1 on T2-weighted plus gadolinium-enhanced T1-weighted MR images, were $71 \%$ (59 of 83 patients), $64 \%$ ( 40 of 63 patients), 95\% (19 of 20 patients), 98\% (40 of 41 patients), and $45 \%$ (19 of 42 patients), respectively, for a nodular tumor margin and $34 \%$ (28 of 83 patients), $27 \%$ (17 of 63 patients), $55 \%$ (11 of 20 patients), $65 \%$ 
(17 of 26 patients), and 19\% (11 of 57 patients), respectively, for a margin consisting of spiculations.

The interobserver agreement expressed by the linear weighted $\mathrm{K}$ statistic with $95 \% \mathrm{Cls}$ was $0.47(95 \% \mathrm{Cl}: 0.31,0.64)$ for T2-weighted MR images and $0.45(95 \% \mathrm{Cl}: 0.25,0.65)$ for combined T2-weighted plus gadolinium-enhanced T1-weighted MR images.

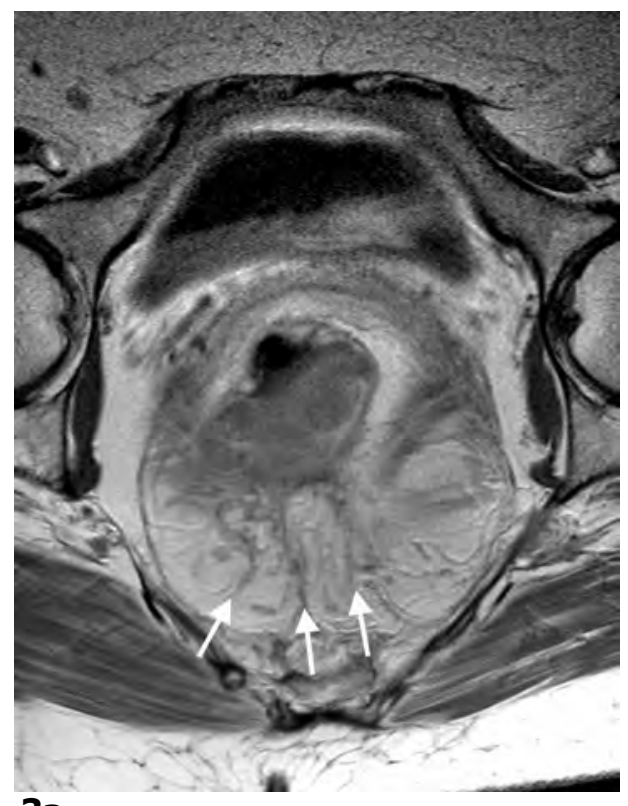

\section{3a}

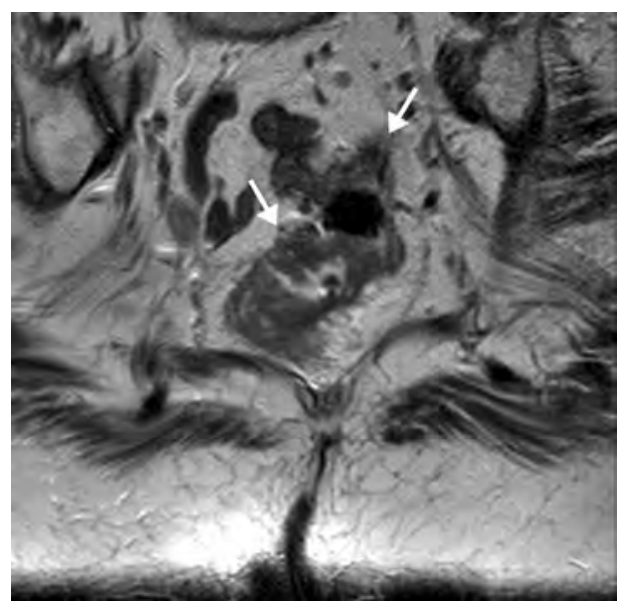

3c

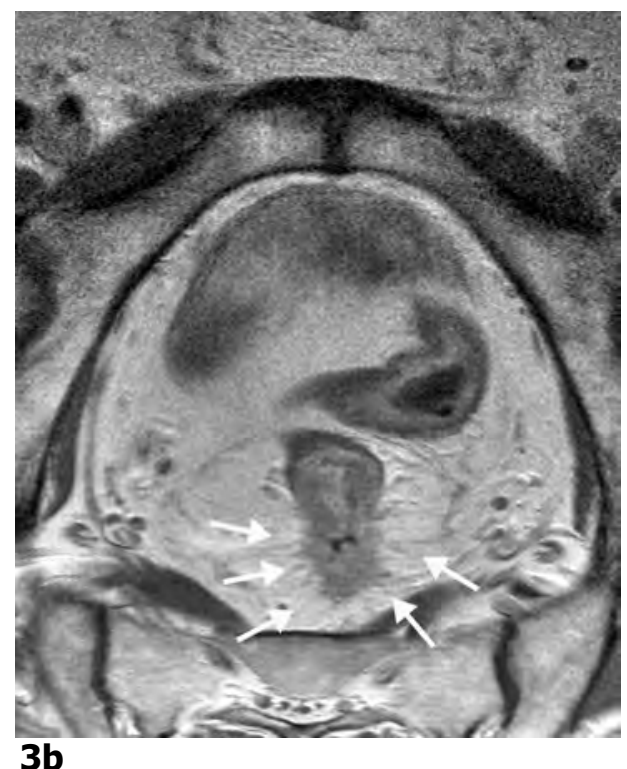

3b

Fig. 3a-c. Difficulty in interpretation of tumor penetration of rectal wall. (a) On this transverse gadolinium-enhanced T1-weighted MR image (612/15), enhancing spiculations (arrows) are seen in mesorectal fat, suggesting tumor penetration of rectal wall (stage T3). However, histologic examination showed that these spiculations consisted of fibrosis without tumor cells (stage T2). (b) On this transverse gadolinium-enhanced T1-weighted MR image $(612 / 15)$ in a different patient, enhancing spiculations (arrows), indistinguishable from those in a, are seen in mesorectal fat. In this case, spiculations consisted of fibrosis and tumor cells (stage T3). Spiculations consisting of fibrosis either with or without tumor cells cannot be discriminated on nonenhanced or gadolinium-enhanced MR images. (c) Clear invasion of mesorectal fat. Coronal T2-weighted turbo SE MR image (3427/150) of tumor with pattern of nodular infiltration (arrows) of mesorectal fat in a different patient. Nodular advancing tumor margin is highly predictive for tumor penetration of rectal wall, as opposed to margin consisting of spiculations $(a, b)$. 


\section{Tumor extension into mesorectal fascia}

The accuracy, sensitivity, specificity, PPV, and NPV of T2-weighted MR images versus combined T2-weighted plus gadolinium-enhanced T1-weighted MR images for the prediction of tumor extension into the mesorectal fascia for observers 1 and 2 are given in table 1.

The $A_{z}$ values with $95 \% \mathrm{Cls}$ of $\mathrm{T} 2$-weighted images only versus T2-weighted plus gadolinium-enhanced T1-weighted images were 0.97 (95\% $\mathrm{Cl}: 0.93,1.01)$ and $0.90(95 \% \mathrm{Cl}: 0.83,0.98)$, respectively, for observer 1 and $0.90(95 \% \mathrm{Cl}: 0.83,0.98)$ and $0.91(95 \% \mathrm{Cl}: 0.84,0.98)$, respectively, for observer 2 (table 1, figure 4). There was no significant difference between $A_{z}$ values for T2-weighted images only and T2-weighted images plus gadolinium-enhanced T1-weighted images for both observers $(P=.052$ for observer 1 and $P=.77$ for observer 2 ) for the prediction of tumor extension into the mesorectal fascia.

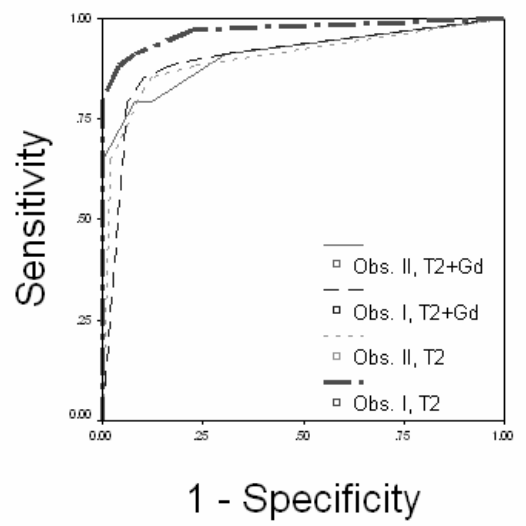

Fig. 4. Diagnostic performance represented by ROC curves of T2-weighted MR sequences (T2) and T2-weighted plus gadolinium-enhanced T1-weighted MR sequences $(T 2+G d)$ for prediction of tumor invasion of mesorectal fascia for observers 1 (Obs. I) and 2 (Obs. II). There was no significant difference $(P>.05)$ between $A_{z}$ values of T2-weighted sequences and combined T2weighted plus gadolinium-enhanced T1-weighted sequences. Diagnostic performance of $\mathrm{MR}$ imaging for prediction of involvement of mesorectal fascia for both observers was high: $A_{z}>0.90$.

There was tumor extension into the mesorectal fascia in 34 patients $(41 \%)$. Thirty-five patients ( $42 \%)$ had a circumferential resection margin larger than $10 \mathrm{~mm}$. On T2-weighted MR images, observers 1 and 2 overestimated two and five tumors, respectively, as tumor extension into the mesorectal fascia and underestimated four and six tumors, respectively. After the addition of gadolinium-enhanced T1-weighted MR imaging to T2-weighted sequences, observers 1 and 2 overestimated five and four tumors and underestimated five and seven tumors, respectively. 
Subgroup analysis of patients with versus those without preoperative radiation therapy, male versus female patients, and age less than 60 years versus age more than 60 years showed that there was only a significant difference between T2-weighted MR sequences and gadolinium-enhanced T1-weighted MR sequences in the group of patients older than 60 years and only for observer $1(P<.05)$. T2-weighted sequences performed significantly better than the combined T2-weighted plus gadolinium-enhanced T1-weighted sequences $(P<.05)$ in this subgroup. The $A_{z}$ values with $95 \% \mathrm{Cls}$, accuracy, sensitivity, specificity, PPV, and NPV of T2-weighted MR images and combined T2-weighted plus gadolinium-enhanced T1-weighted MR images for the prediction of tumor extension into the mesorectal fascia in the group of patients with and those without preoperative radiation therapy for both observers are given in tables 2 and 3.

Analysis of false-positive and false-negative findings showed that there was no specific cause for these failures. Two cases were misinterpreted with both T2-weighted and gadolinium-enhanced T1-weighted imaging because of difficulty in the interpretation of spiculations reaching the mesorectal fascia (figure $5 \mathrm{a}, 5 \mathrm{~b}$ ). These spiculations could consist of fibrosis with or without tumor cells, and the observers were not able to discriminate between the two on images obtained with either sequence. Interpretation difficulties of spiculations in the mesorectal fat obviously do not occur strictly with postradiation MR imaging, as these two specific cases were found in the subgroup of nonirradiated patients. Another reason for the false predictions occurred in a few cases of low anteriorly located bulky tumors. These bulky tumors caused a mass effect on the surrounding pelvic structures, which stretched the fat plane in between (figure $5 c$ ). When there was a normal signal intensity of the surrounding pelvic organs, it was difficult to discriminate mass effect from invasion into this organ. This problem existed on both T2-weighted and gadolinium-enhanced T1-weighted MR images, but for unknown reasons, fewer interpretation problems occurred on T2-weighted images for observer 1 in the patient group older than 60 years.

The interobserver agreement between both observers expressed by the linear weighted $\mathrm{K}$ statistic with $95 \% \mathrm{Cls}$ was $0.75(95 \% \mathrm{Cl}: 0.63,0.86)$ on T2-weighted MR images only and $0.61(95 \% \mathrm{Cl}: 0.48,0.74)$ for the combined T2-weighted plus gadolinium-enhanced T1-weighted MR images. 


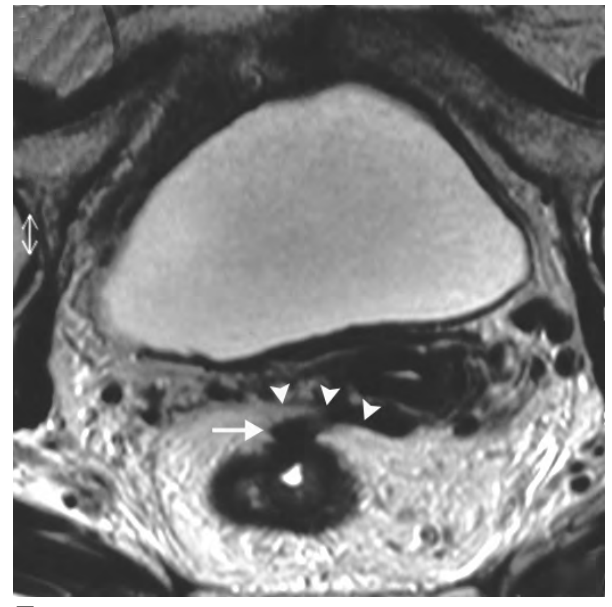

$5 a$

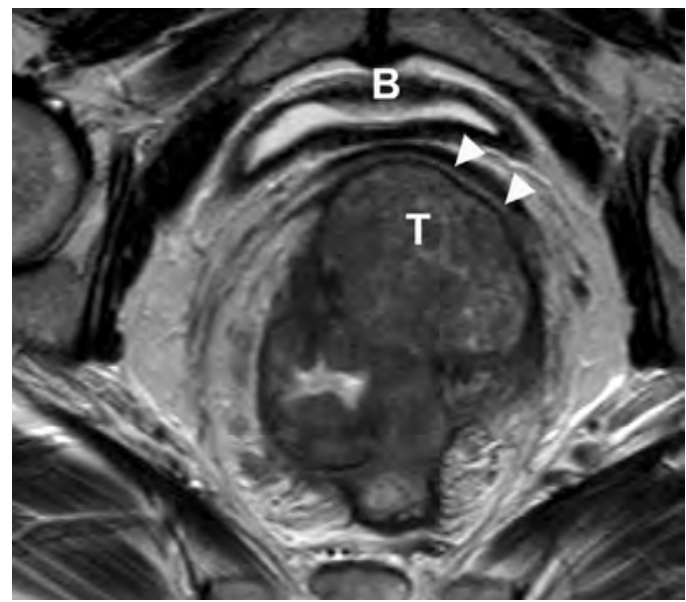

\section{5c}

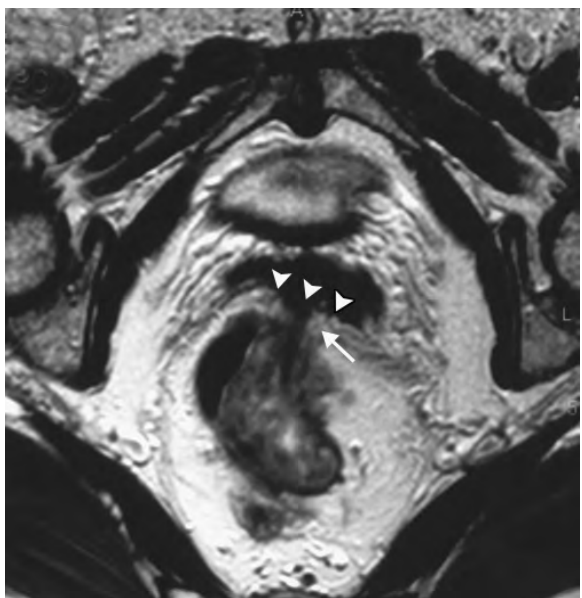

5b

Fig. 5a-c. Difficulty in interpretation of tumor invasion of mesorectal fascia. (a) Transverse T2-weighted turbo SE MR image (3427/150) of tumor with spiculations (arrow) reaching normalappearing mesorectal fascia anteriorly (arrowheads). On contrast-enhanced T1-weighted MR images, these spiculations enhanced (not shown). Histologic examination of surgical specimen showed that these spiculations consisted of fibrosis without tumor cells (no invasion of mesorectal fascia). (b) Transverse T2-weighted turbo SE MR image $(3427 / 150)$ of tumor with spiculations (arrow) reaching normalappearing mesorectal fascia anteriorly

(arrowheads) in a different patient. On contrast-enhanced MR images, these spiculations enhanced (not shown). As opposed to a, spiculations consisted of fibrosis and tumor cells. Contrast-enhanced or nonenhanced MR imaging does not allow differentiation of fibrosis either with or without tumor cells. Therefore, mesorectal fascia must be considered potentially invaded with tumor. (c) Transverse T2-weighted turbo SE MR image (3427/150) of bulky (voluminous) low anteriorly located tumor in a different patient. This tumor $(T)$ causes a mass effect on surrounding pelvic structures (arrowheads indicate vagina) and stretches fat plane in between these structures. When surrounding pelvic organs have normal signal intensity, it can be difficult to differentiate compression from tumor invasion. In this case, histologic examination showed invasion of vagina. $\mathrm{B}=$ bladder. 


\section{Discussion}

\section{T1-weighted MR sequences and rectal wall penetration}

The results of the present study show that the addition of gadoliniumenhanced T1-weighted MR sequences to T2-weighted fast SE MR sequences did not significantly improve the diagnostic accuracy for the prediction of tumor penetration through the rectal wall. The accuracy of MR imaging for the evaluation of tumor penetration through the rectal wall remained moderate for both observers, with accuracies of $69 \%-84 \%$.

A standard protocol for MR imaging of rectal cancer consists of highresolution T2-weighted fast SE sequences (11-12). Different opinions exist about the value of contrast-enhanced MR sequences in rectal cancer staging. In a study of 32 patients, Okizuka et al (13) reported no improvement for $T$ staging after addition of gadolinium-enhanced fat-suppressed MR imaging to conventional T1- and T2-weighted MR imaging. Although in their study, contrast-enhanced MR imaging allowed better tumor detection and delineation, it tends to lead to overstaging of stage $\mathrm{T} 1$ and $\mathrm{T} 2$ tumors because of peritumoral inflammatory reaction. In a study of 106 patients with rectal cancer, Maier et al (14) showed that the tumor could be better identified and delineated by using a double-contrast technique of intravenous gadolinium-based contrast material combined with an enema of ferristene. However, overstaging difficulties occurred in most of the stage T1 tumors (six of 10 tumors) and T2 tumors (13 of 22 tumors). Wallengren et al (16) also used the double-contrast technique and found improved identification of the individual rectal wall layers and tumor delineation. The authors applied a conventional low-spatial-resolution MR technique, however, which may explain the superior performance of their contrast-enhanced sequences. Vogl et al (15) studied 35 patients with superficial tumors and found a high accuracy for stage T1 tumors when a dynamic contrast-enhanced MR technique was applied by using an endorectal coil. Stage T2 and T3 tumors were staged accurately, however, by using nonenhanced T2-weighted sequences only. Contrast-enhanced sequences did not provide additional benefit.

Overall, our findings support the literature that there is at present no real benefit of the use of gadolinium-based contrast material in the staging of rectal cancer with a phased-array MR technique. The moderate accuracy in T staging does not improve with contrast-enhanced sequences. Overstaging as a result of desmoplastic tumor reaction still occurs, and identification of the individual rectal wall layers, particularly the submucosal layer, can still be difficult after contrast enhancement (9,13-14,20). For superficial rectal cancer, either MR imaging with an endorectal coil or endorectal ultrasonography in experienced hands is probably more accurate than MR imaging with a phasedarray coil. 
The moderate accuracy for the prediction of tumor penetration through the rectal wall is in concordance with other studies. In a study of 49 patients, Blomqvist et al (21) showed an accuracy of $76 \%$ for the prediction of tumor penetration through the rectal wall on T2-weighted fast SE MR images. In their study, most of the staging failures were caused by peritumoral tissue reaction that simulated tumor penetration through the rectal wall. The studies in which gadolinium-enhanced MR sequences were used in combination with T2-weighted sequences have the same staging difficulties with accuracies of only $76 \%-82 \%(13-14,20)$.

Difficulties with the interpretation of spiculations in the mesorectal fat on both nonenhanced T2-weighted and contrast-enhanced T1-weighted images were responsible for most of the staging failures in our study. These failures occurred for both observers, independent of their reading experiences. Neither reader was able to distinguish (a) spiculations in mesorectal fat caused by fibrosis that contained tumor cells from (b) spiculations that contained fibrosis only on either nonenhanced or contrast-enhanced MR images. When the pattern of tumor growth through the rectal wall into the mesorectal fat was nodular, however, the PPV increased to $98 \%$, compared with $65 \%$ for a spiculated pattern.

In other fields of MR imaging (ie, musculoskeletal imaging), fatsuppressed MR sequences can help increase the contrast between pathologic and normal surrounding tissues. This is not the case with MR imaging of rectal cancer. Rectal MR imaging involves high natural contrast between the tumor and the perirectal fat. With a fat-suppressed MR technique, the contrast between the hypointense mesorectal fascia and the mesorectal fat disappears, and the anticipated resection margin is difficult to evaluate. The value of fat suppression for the prediction of tumor penetration through the rectal wall is unclear.

A study by Okizuka et al (13) showed that gadolinium-enhanced T1-weighted MR sequences in conjunction with a fat-suppressed technique did not improve the performance of MR imaging for staging of rectal cancer. In their study, as well as in the present study, the main staging problems were caused by desmoplastic tumor reaction, a problem apparently not solved with a fat-suppressed technique.

\section{T1-weighted MR and tumor extension into mesorectal fascia}

Results of the present study show that the addition of gadoliniumenhanced T1-weighted MR imaging does not improve the high accuracy (approximately $90 \%$ ) of T2-weighted fast SE MR imaging for the evaluation of tumor invasion in the mesorectal fascia. The gadolinium-enhanced MR sequences can therefore be omitted, thus saving acquisition time and examination costs and avoiding potential allergic reactions. 
The clinical importance of the relationship of the tumor to the mesorectal fascia should be emphasized. The ideal plane of dissection during a resection of the rectum is along the mesorectal fascia. When the tumor is close to or invading the mesorectal fascia, there is a risk for a positive resection margin with a high risk of local recurrence. Although the T stage is a strong predictor for overall prognosis, for local recurrence, the circumferential resection margin is probably a more important prognostic indicator $(4,22)$. On the basis of the encouraging results of preoperative radiation therapy, a differentiated preoperative treatment according to the risk for local recurrence becomes an attractive option when there is a reliable imaging test. Patients with a low risk could be treated with surgery only, patients with an intermediate risk could be treated with a short course of preoperative radiation therapy, and patients with a locally advanced tumor could be treated with an extensive radiochemotherapy schedule to allow for downsizing.

In several studies, MR imaging has allowed accurate prediction of tumor extension into the mesorectal fascia and the surrounding organs $(12,20,23-25)$. In a study of 76 patients, Beets-Tan et al (20) reported that an involved circumferential resection margin could be predicted with an accuracy of $100 \%$ in 12 stage T4 tumors, and a tumor-free circumferential resection margin could be predicted with an accuracy of more than $90 \%$ in 29 tumors with a wide margin at MR imaging. For the remaining tumors that were close to the mesorectal fascia, a linear regression curve showed that a margin of $1 \mathrm{~mm}$ could be predicted with high certainty when the measured distance between the tumor and the mesorectal fascia at MR was at least $5 \mathrm{~mm}$.

In a study of 26 specimens obtained after total mesorectal excision, Blomqvist et al (24) showed a sensitivity and specificity of $100 \%$ and $61 \%$, respectively, for the prediction of involvement of the circumferential resection margin. In a study of 43 patients with rectal cancer, Bissett et al (12) found an accuracy, sensitivity, and specificity of $95 \%, 67 \%$, and $100 \%$, respectively, for prediction of the circumferential resection margin. In a study of 98 patients, Brown et al (25) showed agreement in 95\% ( $\mathrm{K}=0.81)$ of cases between MR imaging and histologic findings for the prediction of circumferential resection margin. Only in the first-mentioned study by Beets-Tan et al (20) was a gadolinium-enhanced MR sequence added to the high-resolution T2-weighted fast SE MR sequence. The latter three studies reported equally good results without the use of gadolinium-enhanced sequences $(12,24-25)$. The results of the present study support the idea that gadolinium-based contrast material is not essential for the determination of tumor extension into the mesorectal fascia.

On the high-resolution T2-weighted fast SE MR images, even thin structures such as the mesorectal fascia can be well delineated from the mesorectal fat because of the natural difference in signal intensity between the hypointense fascia and the adjacent hyperintense fatty tissues. It is therefore 
relatively easy to estimate the width of the circumferential resection margin after a total mesorectal excision procedure, since it is the width of the hyperintense fat plane between the hypointense mesorectal fascia and the isointense tumor.

Interpretation difficulties remain when a tumor shows a spiculated tumor extension that reaches close to the mesorectal fascia. As shown from the results of our subgroup analysis, this does not occur strictly for postradiation MR imaging but can also occur for primary MR images of nonirradiated rectal cancer, especially of tumors that show an extensive desmoplastic reaction. It is difficult to predict whether the spiculations consist of desmoplastic reaction only or desmoplastic reaction that contains tumor nests. In our experience, even contrast-enhanced dynamic MR sequences will not always depict this microscopic tumor load.

Another type of tumor in which the distance to the mesorectal fascia may be difficult to predict is the low anteriorly located rectal cancer. The distal anterior part of the mesorectum contains less mesorectal fat, and when a tumor breaches the muscular rectal wall anteriorly, it is invariably close to or invades the mesorectal fascia. This is especially so for low bulky tumors, where the fat plane between the tumor and the adjacent structures can be obliterated because of the mass effect of the tumor. In these circumstances, it can be difficult to distinguish compression of adjacent organs (posterior vaginal wall, seminal vesicles, prostate) from tumor invasion into these organs.

When considering the results of the present study, one must keep in mind that the design of the study has some limitations. They relate to the use of an MR technique with an external coil, the reconstruction of the histologic standard of reference in locally advanced rectal cancers, and the inclusion of patients with preoperative radiation therapy. Endorectal coils are more accurate for the staging of superficial tumors than are phased-array coils. The use of an endorectal coil in the present study could have influenced the accuracy for the prediction of tumor penetration through the rectal wall. The results of Vogl et al (15), however, who found no beneficial effect of gadolinium-enhanced MR sequences for staging of stage T2 and T3 tumors with an endorectal coil technique, suggest that the use of different coils would have no substantial effect on the final results of the present study.

The second limitation concerns the histologic standard of reference. The routine histologic evaluation after rectal cancer surgery includes the measurement of the distance from the tumor to the circumferential margin. Most patients underwent standard total mesorectal excision, and the circumferential resection margin coincides with the mesorectal fascia. For the more advanced tumors, however, more extensive surgery was performed, and the distance from the tumor to the mesorectal fascia had to be reconstructed retrospectively by correlating the complete pathologic report with the surgical findings. Although most of these cases were easy to interpret because an 
advanced tumor with invasion in a surrounding structure by definition has invaded the mesorectal fascia, in some patients this could have resulted in errors of the exact histologic distance of the tumor to the mesorectal fascia (most notably after extensive preoperative radiation therapy, see the following).

The third limitation is related to the inclusion of patients after preoperative radiation therapy. Radiation therapy can induce a fibrotic reaction both in normal tissue and in areas of tumor necrosis. For most patients, MR images obtained before radiation therapy were available, and these were used for comparison with histologic findings. When present, areas of clear necrosis and fibrosis at histologic examination were considered former tumor extensions. This may have caused some measurement inaccuracies, as discussed earlier. For the small group of patients that underwent postradiation MR imaging only, interpretation of the images was difficult. Fibrosis that occurs after radiation therapy can sometimes be seen as hypointense spiculations in the perirectal fat. It is difficult to differentiate between postradiation fibrosis, fibrosis that contains viable tumor, and a primary desmoplastic tumor reaction. This can lead to diagnostic errors for the prediction of rectal wall penetration and tumor extension into the mesorectal fascia. To evaluate the effect of the inclusion of patients with preoperative radiation therapy, we performed a subgroup analysis of patients who had undergone radiation therpy and those that had not. Basically, this did not change the results of the study.

The high performance of both readers for the prediction of invasion of the mesorectal fascia with both MR sequences and the high interobserver agreement shows that this can be done with a high accuracy and consistency, as opposed to the prediction of tumor penetration through the rectal wall. This is in concordance with a previous study (20). Striking observations include that even for a more specialized MR reader, it was difficult to predict the $T$ stage, and that a less specialized MR reader performed equally well when predicting invasion of the mesorectal fascia.

\section{Conclusion}

The addition of a gadolinium-enhanced T1-weighted sequence to T2-weighted fast SE MR imaging did not improve the diagnostic accuracy for prediction of tumor penetration through the rectal wall and tumor involvement of the mesorectal fascia. When the protocol for local staging includes highresolution T2-weighted fast SE images, in our opinion, contrast-enhanced sequences can be omitted. The present study confirms previous reports that the performance of phased-array MR imaging is high for the prediction of tumor extension into the mesorectal fascia but only moderate for penetration of the rectal wall, independent of the experience of the reader. 


\section{Footnotes}

Abbreviations: $A_{z}=$ area under the ROC curve, $\mathrm{Cl}=$ confidence interval, $\mathrm{NPV}=$ negative predictive value, $\mathrm{PPV}=$ positive predictive value, $\mathrm{ROC}=$ receiver operating characteristic, $\mathrm{SE}=$ spin echo

\section{References}

1. Sagar P, Pemberton J. Surgical management of locally recurrent rectal cancer. $\mathrm{Br} J$ Surg 1996; 83:293-304.

2. Fielding L, Stewart-Brown S, Dudley $H$. Surgeon-related variables and the clinical trial. Lancet 1978; 2:778-779.

3. Quirke $P$, Durdey $P$, Dixon $M$, et al. Local recurrence of rectal adenocarcinoma due to inadequate surgical resection: histopathological study of lateral tumour spread and surgical excision. Lancet 1986; 2:996-999.

4. Quirke P, Dixon $M$. The prediction of local recurrence in rectal adenocarcinoma by histopathological examination. Int J Colorectal Dis 1988; 3:127-131.

5. Heald R, Ryall R. Recurrence and survival after total mesorectal excision for rectal cancer. Lancet 1986; 1:1479-1482.

6. Improved survival with preoperative radiotherapy in resectable rectal cancer: Swedish Rectal Cancer Trial. N Engl J Med 1997; 336:980-987.

7. Colorectal Cancer Collaborative Group. Adjuvant radiotherapy for rectal cancer: a systematic overview of 8,507 patients from 22 randomised trials. Lancet 2001; 358:12911304.

8. Kapiteijn E, Marijnen C, Nagtegaal I, et al. Preoperative radiotherapy combined with total mesorectal excision for resectable rectal cancer. $\mathrm{N}$ Engl J Med 2001; 345:638-646.

9. Laghi A, Ferri M, Catalano C, et al. Local staging of rectal cancer with MRI using a phased array body coil. Abdom Imaging 2002; 27:425-431.

10. Wiggers $T$, van de Velde $C$. The circumferential margin in rectal cancer: recommendations based on the Dutch Total Mesorectal Excision study. Eur J Cancer 2002; 38:973-976.

11. Brown G, Richards C, Newcombe R, et al. Rectal carcinoma: thin-section MR imaging for staging in 28 patients. Radiology 1999; 211:215-222.

12. Bissett I, Fernando $C$, Hough D, et al. Identification of the fascia propria by magnetic resonance imaging and its relevance to preoperative assessment of rectal cancer. Dis Colon Rectum 2001; 44:259-265.

13. Okizuka H, Sugimura K, Yoshizako T, et al. Rectal carcinoma: prospective comparison of conventional and gadopentetate dimeglumine enhanced fat-suppressed MR imaging. J Magn Reson Imaging 1996; 6:465-471.

14. Maier A, Kersting-Sommerhoff B, Reeders J, et al. Staging of rectal cancer by doublecontrast MR imaging using the rectally administered superparamagnetic iron oxide contrast agent ferristine and IV gadodiamide injection: results of a multicenter phase II trial. J Magn Reson Imaging 2000; 12:651-660.

15. Vogl T, Pegios W, Mack M, et al. Accuracy of staging rectal tumors with contrastenhanced transrectal MR imaging. AJR Am J Roentgenol 1997; 168:1427-1434.

16. Wallengren N, Holtås S, Andrén-Sandberg $\AA$, et al. Rectal carcinoma: double contrast MR imaging for preoperative staging. Radiology 2000; 215:108-114.

17. Hanley J, McNeil B. A method of comparing the areas under receiver operating characteristic curves derived from the same cases. Radiology 1983; 148:839-843.

18. Cohen J. Weighted kappa: nominal scale agreement with provision for scaled disagreement or partial credit. Psychol Bull 1968; 70:213-230. 
19. Landis J, Koch G. The measurement of observer agreement for categorical data. Biometrics 1977; 33:159-174.

20. Beets-Tan R, Beets $G$, Vliegen $R$, et al. Accuracy of magnetic resonance imaging in prediction of tumour-free resection margin in rectal cancer surgery. Lancet 2001; 357:497-504.

21. Blomqvist L, Holm T, Rubio $C$, et al. Rectal tumours: MR imaging with endorectal and/or phased-array coils, and histopathological staging on giant sections. Acta Radiol 1997; 38:437-444.

22. Nagtegaal I, Marijnen C, Kranenbarg E, et al. Circumferential margin involvement is still an important predictor of local recurrence in rectal carcinoma: not one millimeter but two millimeters is the limit. Am J Surg Pathol 2002; 26:350-357.

23. Beets-Tan R, Beets G, Borstlap A, et al. Preoperative assessment of local tumor extent in advanced rectal cancer: CT or high resolution MRI? Abdom Imaging 2000; 25:533-541.

24. Blomqvist L, Rubio C, Holm T, et al. Rectal adenocarcinoma: assessment of tumour involvement of the lateral resection margin by MRI of resected specimen. $\mathrm{Br} J$ Radiol 1999; 72:18-23.

25. Brown G, Radcliffe A, Newcombe R, et al. Preoperative assessment of prognostic factors in rectal cancer using high resolution magnetic resonance imaging. $\mathrm{Br} J$ Surg 2003; 90:355- 364 . 


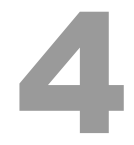

The accuracy of Multi-detector row CT for the assessment of tumor invasion of the mesorectal fascia in primary rectal cancer

RF Vliegen, RC Dresen, GL Beets, AW Daniels-Gooszen, AG Kessels, J M van Engelshoven, RG Beets-Tan.

Abdominal Imaging 2008 (in press). 


\section{Abstract}

Objective: To evaluate the accuracy of Multi-Detector row CT (MDCT) for the prediction of tumor invasion of the mesorectal fascia (MRF).

Materials and methods: Thirty-five patients with primary rectal cancer underwent preoperative staging MRI and MDCT. The tumor relationship to the MRF, expressed in 3 categories (1-tumor free MRF=tumor distance $\geq 1 \mathrm{~mm}$; 2-threatened=distance $<1 \mathrm{~mm} ; 3$-invasion=distance $0 \mathrm{~mm}$ ) was determined on CT by two observers at patient level and at different anatomical locations. A third expert reader evaluated the MRF tumor relationship on MRI which served as reference standard. Receiver Operating Characteristic curves (ROC-curves) and Areas Under these Curves (AUC) were calculated. The interobserver agreement of CT was determined by using linear weighted kappa statistics.

Results: The AUC of CT for MRF invasion was 0.71 for observer 1 and 0.62 for observer 2 . The inter-observer agreement was kapa $=0.34$. The performance of $\mathrm{CT}$ at mid-high rectal levels was statistical significant better compared to low anterior (obs. 1 : $A U C=0.88$ vs 0.50 ; obs 2 : $A U C=0.84$ vs 0.31 ; $\mathrm{p} \leq 0.040)$.

Conclusion: MDCT has a poor accuracy for predicting MRF invasion in low-anterior located tumors. The accuracy of CT significantly improves for tumors in the mid-high rectum. There is a high inconsistency among readers. 


\section{Introduction}

The prognosis of patients with rectal cancer has improved since the introduction of total mesorectal excision (TME) surgery (1-3). Using this surgical technique the mesorectal compartment including the rectum and perirectal fat is completely excised by sharp dissection along the mesorectal fascia (1). Additionally, large randomized trials have shown that neo-adjuvant therapy improves local tumor control even further, regardless of optimized surgical techniques (3-4). The advances in rectal cancer treatment have provoked differentiated neo-adjuvant treatment strategies based on anatomical preoperative identifiable risk factors for local tumor recurrence as can be visualized with MRI (5). One of the most important risk factors is the tumor relationship to the mesorectal fascia (MRF) which actually defines the surgical circumferential resection margin (CRM) in total mesorectal excision (TME) surgery (6-7). Long courses of neo-adjuvant chemoradiation have emerged as the preferential treatment of patients with anticipated tumor invasion of the MRF on MRI in order to downstage/ downsize the tumor and to obtain tumor free resection margins (5).

MRI has become an integral part of the diagnostic work-up of patients with rectal cancer due to its proven efficacy to determine the tumor relationship to the mesorectal fascia $(5,8-11)$. However, the moderate availability (12) and the higher cost of MR on one hand, and improved Multi-Detector row CT (MDCT) on the other hand, have revived the discussion whether to use CT or MRI for rectal cancer staging. With these state of the art CT technique it has become feasible to visualize the mesorectal fascia and to perform a quick one stop shop examination of the whole abdomen including distant metastasis. At present, only a few MDCT studies have been published on the subject of rectal cancer staging $(13-16,18)$. To our knowledge, two studies focussed on the prediction of tumor invasion of the mesorectal fascia $(13,17)$. One study with MDCT suggested results equal to MRI, and the other with conventional CT suggested results inferior to MRI. The question on the accuracy of MDCT remains open.

The application of chemoradiation as the prefered treatment of locally advanced rectal cancer has created a major methodological problem for the evaluation of the staging accuracy of new imaging techniques. Because of the tumour downsizing the traditional gold standard of histology is no longer valid. Subgroup analysis of patients treated with short-courses of 5x5 Gy radiotherapy (no downstaging or downsizing effects) or without neo-adjuvant therapy obviously introduces a selection bias of small tumors in which imaging of tumour invasion of the mesorectal fascia cannot be assessed because there is no invasion. 
Therefore, the aim of the present study was to evaluate the accuracy of MDCT for the prediction of tumor invasion of the mesorectal fascia with MRI as reference standard.

\section{Materials and methods}

\section{Patients}

Institutional approval was obtained for this retrospective study. The records of a cohort of consecutive patients with biopsy proven primary rectal cancer were searched for patients who underwent both MRI and MDCT imaging at the University Hospital Maastricht between J une 2004 and J une 2006. The standard workup for patients with a rectal cancer includes a pelvic MRI. Some patients received a MDCT because of preoperative planning of radiotherapy. Inclusion criteria were availability of both MRI and contrast enhanced CT examinations of good quality prior to the application of any neo-adjuvant therapy. In total 35 patients met these inclusion criteria and were enrolled in the present study. Relevant clinical information such as type of neo-adjuvant therapy, type of operation, radiologic and histologic reports were recorded. There were 23 men and 12 women. The mean age of the patients was 68 years (range 44-85 years). The mean time between the MRI and CT examination was 24 days (range 1-49 days).

\section{Technique}

All CT studies were performed on a 16 -slice CT system (SOMATOM ${ }^{\circledR}$ Sensation 16, Siemens, Erlangen, Germany). The scan protocol comprised a slice collimation of $16 \times 0.75 \mathrm{~mm}$, table feed/rotation: $15.0 \mathrm{~mm}$, rotation time 0.5 seconds, reconstruction increment $5 \mathrm{~mm}, 5 \mathrm{~mm}$ reconstructed slice thickness. All patients received $120 \mathrm{ml}$ of nonionic IV contrast agent (Iobitridol $350 \mathrm{mg}$, Xenetix ${ }^{\circledR}$, Guerbet, France) at a flow rate of $3 \mathrm{ml} / \mathrm{sec}$ and oral contrast (Telebrix gastro ${ }^{\circledR}$, Guerbet). The images of the pelvis were obtained in the porto-venous contrast phase (scan delay 60 seconds). None of the patients received a contrast enema or bowel relaxation.

MR imaging was performed on a 1.5 Tesla system (Gyroscan, Powertrack 6000 NT, Philips Medical Systems, The Netherlands) using a phased array sense-cardiac coil. The scan protocol consisted of a T2-weighted sequence obtained in three orientations (axial, sagittal and coronal): turbo(fast)spin echo, $T R=3427 / T E=150 \mathrm{msec}$, echo train length of $25,4 \mathrm{~mm}$ slice thickness, $0.8 \mathrm{~mm}$ gap, 6 signal averages, $175 \times 256$ matrix, $20 \mathrm{~cm}$ FOV. None of the patients received Gadolinium contrast enhanced sequences, fat suppression tecniques, rectal contrast enema or bowel relaxation. 


\section{Image evaluation}

The CT examinations were read by two observers who were blinded to each other and to the clinical and MRI results. Observer 1 was a dedicated abdominal radiologist with more than 8 years of experience in reading pelvic CT and MRI (R.V.). Observer 2 was a general radiologist with more than 5 years experience in reading cross sectional imaging (A.D.G). The MRI examinations were assessed by a third observer with more than 12 years experience in rectal cancer MRI and who has read over 1500 MRIs of rectal cancer cases (R.B.T).

Observer 1 and 2 assessed the relationship between the rectal tumor and the mesorectal fascia on the CT images and the third observer on MRI. The mesorectal fascia (MRF) was defined as the fine linear structure surrounding the mesorectal compartment, hypo-intense on T2-weighted MR images and iso-intense to muscle on CT. The relation of the rectal tumor to the mesorectal fascia (MRF) was expressed in 3 discrete categories: 1=tumor free MRF defined as a measured distance between the tumor periphery and the MRF of $\geq 1 \mathrm{~mm}$; $2=$ tumor threatened MRF defined as a distance of $<1 \mathrm{~mm} ; 3=$ tumor invaded MRF defined as a distance of $=0 \mathrm{~mm}$ between the tumor and the mesorectal fascia $(9,11)$. All image evaluations and measurements were performed on a viewing station.

Specific anatomical locations of the MRF were individually evaluated for presence of rectal cancer and, if present, the tumor relation to the MRF was assessed according to the previous described criteria 1-3. Anatomical locations were defined as:

1-Low anterior (anterior mesorectal quadrant at the level of the prostate and vagina)

2-Low lateral left, posterior and lateral right (lateral and posterior mesorectal quadrants at the level of the pelvic floor upto the level of the obturator muscles and low sacrum)

3-Mid anterior in males (anterior mesorectal quadrant at the level of the seminal vesicles)

4-Mid-high lateral left, posterior and lateral right (lateral and posterior mesorectal quadrants at the level of the obturator muscles upto the piriform muscles and hig sacrum)

\section{Statistical analysis}

The CT findings at patient level and at the level of different anatomical locations were compared with the corresponding MRI findings, serving as the reference standard. Receiver operating characteristic curves (ROC curves) were constructed and areas under the curves (AUC) were calculated by using the scored categories 1-3. The difference in performance of MDCT for the prediction of MRF invasion for different anatomical locations was analysed by 
means of comparison of the corresponding AUC and the calculation of a critical ratio $z$ according to the method of Hanley (19). For this analysis, only anatomical locations with abnormal rectal walls on MRI were included [19]. A $p$ value of $<0.05$ was considered as statistical significant. Accuracy, sensitivity, specificity, positive predictive value (PPV) and negative predictive value (NPV) of $\mathrm{CT}$ for the prediction of tumor invasion of the MRF was calculated for both observers by dichotomization of the scored discrete categories 1-3 (cutoff between category 1 vs 2-3). The inter-observer agreement for CT was determined by using linear weighted kappa statistics based on the scored discrete categories 1-3 (20). Statistical analysis was performed by using the software package SPSS for Windows ${ }^{\circledR}$, release 11.5 (Chicago, IL).

\section{Results}

\section{Reference standard MRI vs clinical results}

Fifteen of 35 patients had locally advanced rectal tumors with tumor invasion of the MRF on MRI. All of these 15 patients were treated with long courses of chemoradiation followed by TME in 3 of 15, APR (abdominal perineal resection) in 2 of 15 , pelvic exenteration with or without sfincter preservation in 8 of 15 and no operation in 2 of 15 patients. The remaining 20 of 35 patients had a tumor free MRF on MRI. Fifteen of these 20 patients received short course radiotherapy ( $5 \times 5 \mathrm{~Gy}$ ) followed by TME. Histologic evaluation of the surgical specimen showed in all of these 15 patiens a tumor free MRF. The remaining 5 of 20 patients with a predicted tumor free mesorectal fascia on MRI received long courses of chemoradiation based on adverse prognostic risk factors such as advanced nodal disease and distal location. Three of these 5 patients were treated with TME, 1 of 5 with APR and 1 of 5 received no operation.

\section{Performance of CT at patient level}

Staging failures of CT for the prediction of tumor invasion of the MRF occurred in 12/35 (34\%) patients for observer 1 and 16/35 (46\%) for observer 2 (table 1 ). The majority of these failures were based on the overstaging of the tumor relation to the MRF (obs 1: $10 / 12=83 \%$; obs 2: $13 / 16=81 \%$ ). The area under the ROC curve (AUC), accuracy, sensitivity, specificity, PPV and NPV are displayed in table 2. The inter-observer agreement expressed by the linear weighted kappa was $0.34(95 \% \mathrm{Cl}: 0.04,0.64)$. 
Table 1. CT vs reference standard MRI for the prediction of the tumor relationship to the MRF at patient level.

\section{Free MRF}

Invaded MRF*

\section{CT observer 1:}

Free

Threatened

Invaded

$\begin{array}{rr}10 & 2 \\ 2 & 1 \\ 8 & 12\end{array}$

\section{CT observer 2:}

Free

Threatened

Invaded

\section{7}

2

11
3

12

Total: 20 15

Note. Free $M R F=$ measured distance between tumor and MRF of $\geq 1 \mathrm{~mm}$. Tumor threatened $\mathrm{MRF}=$ distance between tumor and MRF of $<1 \mathrm{~mm}$. Invaded MRF=tumor in contact with MRF; * none of the patients had a tumor threatened MRF on MRI.

Table 2. Performance of $\mathrm{CT}$ for the prediction of tumor invasion of the MRF at patient level.

\begin{tabular}{lll}
\hline & Observer 1 & Observer 2 \\
\hline AUC $(95 \% \mathrm{Cl})$ & $0.71(0.54,0.88)$ & $0.62(0.43,0.81)$ \\
Accuracy & $66 \%(23 / 35)$ & $54 \%(19 / 35)$ \\
Sensitivity & $87 \%(13 / 15)$ & $80 \%(12 / 15)$ \\
Specificity & $50 \%(10 / 20)$ & $35 \%(7 / 20)$ \\
PPV & $57 \%(13 / 23)$ & $48 \%(12 / 25)$ \\
NPV & $83 \%(10 / 12)$ & $70 \%(7 / 10)$ \\
\hline
\end{tabular}

Note. $A U C=$ Area under the receiver operating characteristic curve; $(95 \% \mathrm{Cl})=95 \%$ confidence interval;

$\mathrm{PPV}=$ positive predictive value; $\mathrm{NPV}=$ negative predictive value.

\section{Analysis of different anatomical locations}

The results of $\mathrm{CT}$ for the evaluation of tumor invasion at different anatomical locations are shown in table 3 . There was a statistical significant difference between the performance of $\mathrm{CT}$ at the mid-high lateral-posterior rectum compared to the low anterior rectum for both observers (obs.1: $\mathrm{AUC}=0.88$ vs $0.50, p=0.040 ;$ obs $2: \mathrm{AUC}=0.84$ vs $0.31, p=0.003$. Overstaging occurred in $87 \%(27 / 31)$ of the staging failures of CT for observer $1,83 \%(33 / 40)$ for observer 2 and were mainly observed at the lower rectum (figure 1 and 2). CT failed to visualize the rectal tumor at 1 of 4 anatomical locations with tumor invasion of the MRF for observer 1 and 5 of 7 for observer 2 (figure 3). Aditionally, observer 1 predicted in 7 of 120 
anatomical locations with a normal rectal wall on MRI tumor invasion of the MRF and in 11 of 120 for observer 2 (figure 4).

Table 3. Performance of $\mathrm{CT}$ for the prediction of tumor invasion of the MRF at different anatomical locations.

\begin{tabular}{|c|c|c|c|c|}
\hline & \multicolumn{2}{|c|}{ MRF invasion } & \multirow{2}{*}{$\begin{array}{c}\text { Observer } 1 \\
\text { AUC }(95 \% \mathrm{CI})\end{array}$} & \multirow{2}{*}{$\begin{array}{c}\text { Observer } 2 \\
\text { AUC }(95 \% \mathrm{CI})\end{array}$} \\
\hline & - & + & & \\
\hline \multicolumn{5}{|l|}{ locations: } \\
\hline Low anterior & 3 & 8 & $0.50(0.10,0.90) *$ & $0.31(0.00,0.64) *$ \\
\hline Low lat-post & 32 & 3 & $0.78(0.60,0.97)$ & $0.58(0.19,0.96)$ \\
\hline Mid anterior & 10 & 7 & $0.71(0.46,0.97)$ & $0.66(0.35,0.97)$ \\
\hline Mid-high lat-post & 77 & 8 & $0.88(0.73,1.00) *$ & $0.84(0.66,1.00) *$ \\
\hline All locations** & 122 & 26 & $0.82(0.73,0.92)$ & $0.70(0.56,0.84)$ \\
\hline
\end{tabular}

Note. ** locations with normal rectal wall on MRI were excluded from analysis (120/268). $A U C=$ area under the ROC curve with $95 \%$ confidence interval; -=number of cases with a tumor free MRF predicted on MRI; $+=$ number of cases with a tumor threatened or invaded MRF; * statistical significant difference $p \leq 0.04$.

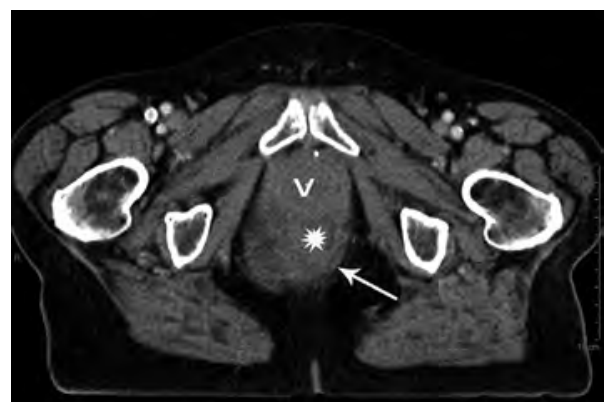

$1 \mathbf{a}$

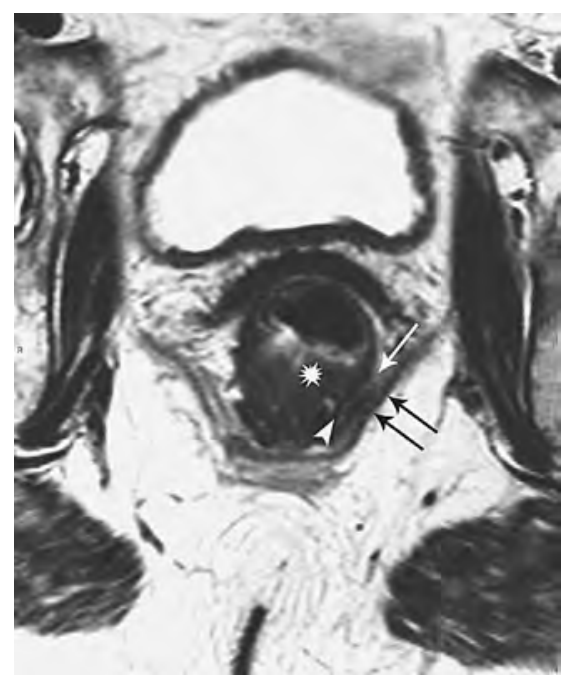

1b

Fig. 1a-b. Poor anatomical detail on CT leading to overestimation of tumor invasion of the MRF in distal rectal tumors. (a) Axial MS-CT image of distal rectal cancer. The tumor (asterisk) is difficult to delineate and no fat pad can been seen between the tumor and the pelvic floor (arrow) suggesting invasion of the MRF. (b) Axial T2-weighted MR image at the same level shows a tumor free MRF represented by a partial intact muscular rectal wall layer (arrowhead) and a minimal fat pad (arrow) inbetween the the tumor and the pelvic floor muscles (double arrow). 


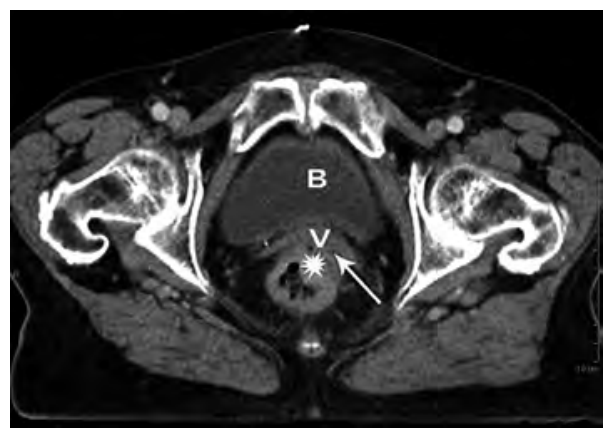

2a

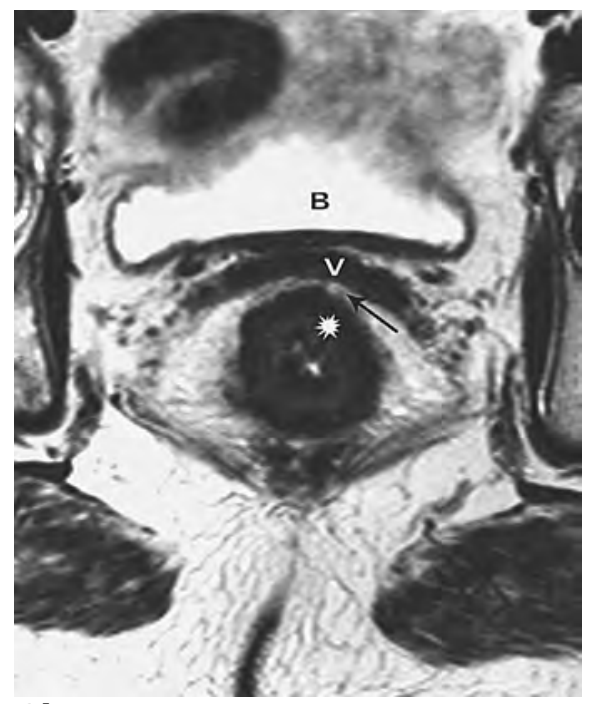

\section{2b}

Fig. 2a-b. Another example of poor anatomical detail on CT causing overestimation of MRF invasion in difficult anatomical regions. (a) Axial MS-CT image of distal rectal cancer (asterisk) showing tumor invasion of the anterior MRF (arrow) and vagina (v). (b) Axial T2-weighted MR images at the same level shows a tumor free anterior MRF and vagina (v) represented by a well appreciated fat pad (arrow) between the tumor (asterisk) and the anterior MRF.

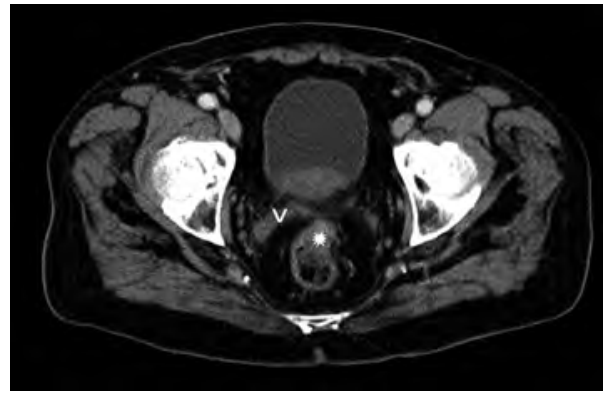

$3 a$

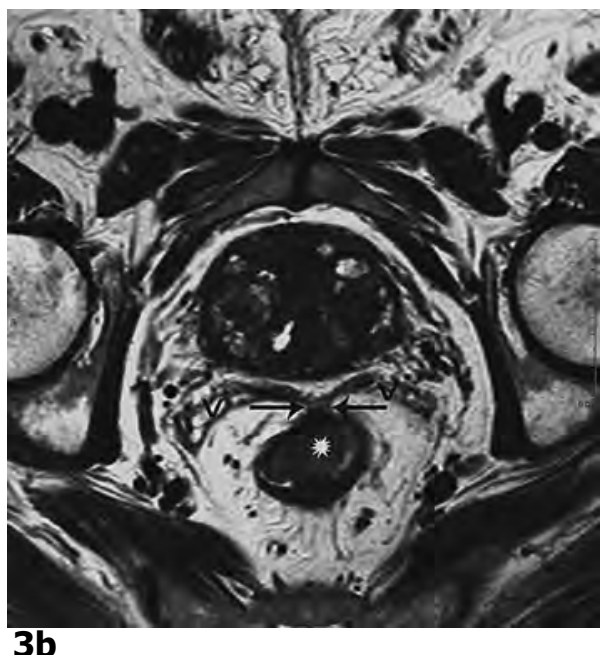

Fig. 3a-b. Difficult visualisation of the tumor localisation on CT leading to underestimation of tumor invasion of the MRF. (a) Axial MS-CT image. The rectal tumor located in the anterior rectum (asterisk) at the level of the seminal vesicles (v) is difficult to appreciate due to partial collaps of the rectal lumen and suboptimal soft tissue contrast resolution. (b) Axial T2-weighted MR image at the same level illustrates an optimal visualisation of the tumor in the rectal wall due high anatomical detail. Also the tumor spread into the anterior MRF (arrows) is well appreciated because of the high soft tissue contrast resolution ( $V=$ seminal vesicles). 


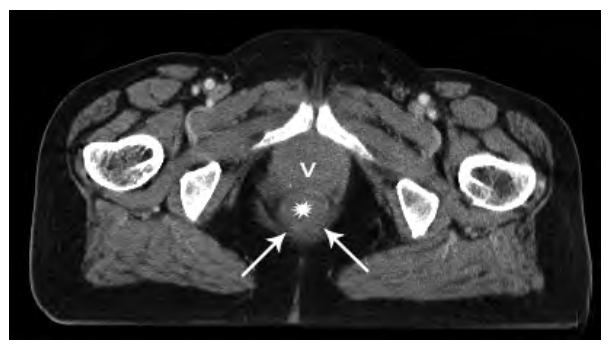

$4 a$

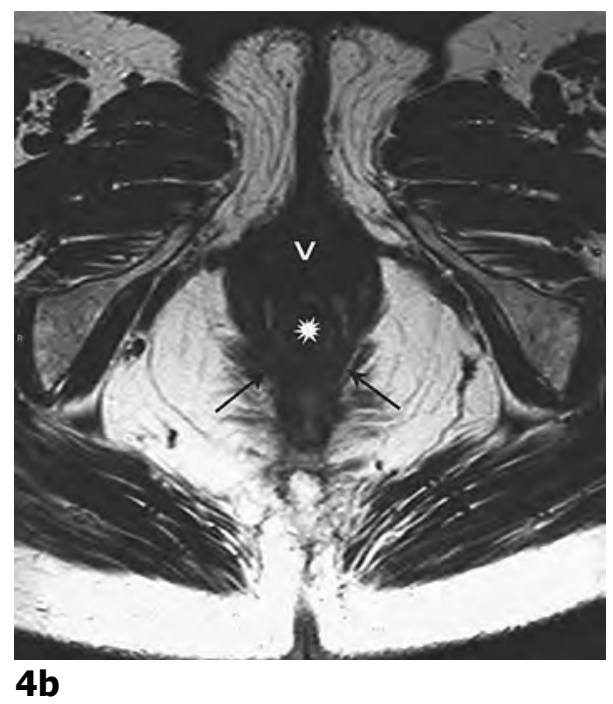

Fig. 4a-b. Normal rectal wall staged as tumor invasion of the MRF on CT due to insufficient anatomical detail. (a) Axial MS-CT image suggest a thickened rectal wall interpreted as distal tumor (asterisk) contacting the pelvic floor (arrows) and vagina (v). (b) Axial T2-weighted MR image at the same level clearly depicts a normal rectal wall (asterisk) as well as surrounding anatomy (arrows=pelvic floor; $v=$ vagina).

\section{Discussion}

The present study evaluated the accuracy of MDCT for the prediction of tumor invasion of the mesorectal fascia with MRI as reference standard. We found a poor accuracy (54-66\%), AUC (0.62-0.71) and high inconsistency among observers for the prediction of tumor invasion of the mesorectal fascia (MRF). Evaluation of the staging accuracy of $\mathrm{CT}$ at different anatomical locations showed very poor AUCs (0.31-0.50) for low anterior tumors but the performance significantly improved for mid-high lateral-posterior located rectal tumors (AUC: $0.84-0.88 ; p \leq 0.04$ ).

Some studies on the performance of MDCT for staging of rectal cancer have focussed on the prediction of $\mathrm{T}$ stage (TNM-staging classification) $(13-16,18)$. Matsuoka et al. found in a study of 21 patients comparing MDCT with MRI an agreement of $95 \%$ between MDCT and histology, with no difference between MDCT and MRI. Kulinna et al. reported an accuracy of $86 \%$ in a study of 92 patients (15). The results of Sinha et al. showed an accuracy of $87 \%$ for the prediction of T stage in 69 patients (18). The study of Taylor et al. adressed the clinical important prediction of the tumor relationship to the mesorectal fascia and reported a poor agreement between MDCT, MRI and histology (kappa 0.06-0.15) in 42 patients treated with a short course of radiotherapy (5x5 Gy) or surgery only (13). The negative predictive value of both MRI and CT was good ( $82 \%$ and $85 \%$ ), but both modalities had a very 
low positive predictive value ( $25 \%$ and $31 \%)$. These results confirm our findings of overstaging with $\mathrm{CT}$, although the overstaging of MRI is rather surprising considering the high accuracy reported in several previous studies (8-11). The adopted wide cutoff $(<5 \mathrm{~mm})$ for the definitition of tumor threatening and invasion of the MRF might have been responsible for this substantial overstaging (11). Wolberink et al report in a retrospective study of 125 with and without a short course of radiotherapy on the value of conventional CT in predicting mesorectal fascia invasion (17). The area under the curve of the ROC curve ranged between 0.697 and 0.813 , and is comparable to the present study. The sensitivity in this study was just below $50 \%$, and the majority of false negatives occurred in tumors located in the distal anterior rectum. The present study confirms the difficulty in interpreting these tumors even on MDCT.

Our results showed a general overstaging of the tumor relation to the MRF on CT. The main causes were: a poor localisation of the tumor in the rectum, leading to misinterpretation of a thickened wall as tumor and vice versa; inability to detect a (partial) intact muscular rectal wall; and a poor appreciation of a small fat pad inbetween the tumor and the MRF. Despite major progress of image quality with the Multi-detector row technique, its poor soft tissue contrast resolution compared to MRI remains (21-22). High anatomical detail is essential for the evaluation of distal tumors as we experienced in the majority of our CT staging failures. Tumor penetration of the distal rectal wall almost directly results in tumor invasion of the MRF due to its close relation (23). This close relation of anatomy restricts the application of rectal inflation by contrast enemas which has been generally used in MDCT studies in order to better appreciate the tumor location (14-16). The inflation of the rectum causes stretching of the perirectal fat plane and reduces the distance to the mesorectal fascia leading to overestimation of tumor invasion (24).

The soft tissue contrast resolution appeared to be far less critical in the mid-upper rectum, reflected by the high AUCs (0.84-0.88) for both observers, significantly better than for low anterior rectal tumors. The presence of a broad non truncated fat manchet surrounding the rectum at these levels gives sufficient contrast between tumor and mesorectal fascia.

A major point of concern is the poor inter-observer agreement (kappa $=0.34$ ) of CT despite the use of objective criteria for tumor invasion of the MRF. High consistency among observers with different levels of experience is one of the most important pre-requisites for the general acceptance of a staging technique. The consistent results among observers and repeatedly reported high accuracy for the prediction of MRF invasion has contributed to the general acceptance of MRI as the most appropriate selection tool for neo-adjuvant and surgical therapy $(8-9,25)$. 
When considering the results of the present study, one must keep in mind that the design has some limitations.We used reconstructed CT slices of $5 \mathrm{~mm}$ which were evaluated in the axial plane only. The use of thinner slices might improve the prediction of MRF invasion in difficult anatomical regions, however, this has never been studied. The only reports on this subject have shown improved T staging by multi planar reconstruction (MPR) $(14,18)$. The main limitation is related to the use of MRI as the reference standard, rather than histology. As argued in the introduction this has become virtually impossible since patients with a tumor that invades or threatens the mesorectal fascia are currently treated with long courses of chemoradiation. Studying only patients who are treated with surgery only or a short course of preoperatieve radiotherapy creates selection bias of non locally advanced rectal cancer, and excludes those patients who are most at risk for involvement of the mesorectal fascia. $(13,16)$. Other authors have compared imaging after neo-adjuvant chemoradiation with histology, but this is problematic because of the difficulties in interpreting radiofibrosis, and does not give an answer to the question of the relation of the tumor to the mesorectal fascia before chemoradiation $(9,26)$. MRI is the only modality that has been extensively evaluated for the prediction of tumor invasion of the mesorectal fascia and several studies have agreed upon its high accuracy $(8-11,25)$. Based on the current evidence, we feel that MRI gives the best available representation of the tumor extension to the mesorectal fascia prior to chemoradiation and therefore could serve as a reference standard.

\section{Conclusion}

MDCT has a poor accuracy for the identification invasion of the mesorectal fascia in low anterior located tumors. The accuracy of CT significantly improves for tumors located in the mid to high rectum. There is a high inconsistency among readers.

\section{References}

1. Heald R. Local recurrence of rectal cancer. Dis Colon rectum 1987; 30:572.

2. Enker W. Total mesorectal excision-the new golden standard of surgery for rectal cancer. Ann Med 1997; 29:127-133.

3. Kapiteijn E, Marijnen C, Nagtegaal I, et al. Preoperative radiotherapy combined with total mesorectal excision for resectable rectal cancer. N Engl J Med 2001; 345:638-646.

4. Sauer $R$, Beckers $H$, Hohenberger $W$, et al Preoperative vs postoperative chemoradiotherapy for rectal cancer. N Engl J Med 2004; 351:1731-1740.

5. Valentini V, Glimelius B, Minsky B, et al. The multidisciplinary rectal cancer treatment: main convergences, controversial aspects and investigational areas which support the need for an European consensus. Radiother Oncol 2004; 76:241-250.

6. Quirke $P$, Dixon $M$. The prediction of local recurrence in rectal adenocarcinoma by histopathological examination. Int J Colorectal Dis 1988; 3:127-131. 
7. Nagtegaal I, Marijnen C, Kranenbarg E, et al. Circumferential margin involvement is still an important predictor of local recurrence in rectal carcinoma: not one millimeter but two millimeters is the limit. Am J Surg Pathol 2002; 26:350-357.

8. Beets-Tan $R$, Beets $G$, Vliegen $R$, et al. Accuracy of magnetic resonance imaging in prediction of tumour-free resection margin in rectal cancer surgery. The Lancet 2001; 357:497-504.

9. MERCURY study group. Diagnostic accuracy of preoperative magnetic resonance imaging in predicting curative resection of rectal cancer: prospective observational study. BMJ 2006; 333:779-790.

10. Lahaye $M$, Engelen $S$, Nelemans $P$, et al. Imaging for predicting the risk factors--the circumferential resection margin and nodal disease--of local recurrence in rectal cancer: a meta-analysis. Semin Ultrasound CT MR 2005; 26:259-268.

11. Brown G, Radcliffe A, Newcombe R, et al. Preoperative assessment of prognostic factors in rectal cancer using high-resolution magnetic resonance imaging. $\mathrm{Br} J$ Surg 2003; 90:355-364.

12. Taylor A, Sheridan M, Mc Gee S, et al. Pre-operative staging of rectal cancer by MR imaging: results of a UK survey. Clin Radiol 2005; 60:579-586.

13. Taylor A, Slater A, Mapstone N, et al. Staging rectal cancer: MRI compared to MDCT. Abdom Imaging 2007; 32:323-327.

14. Kulinna C, Eibel R, Matzek W, et al. Staging of rectal cancer: diagnostic potential of multiplanar reconstructions with MDCT. Am J Roentgenol 2004; 183:421-427.

15. Matsuoka $\mathrm{H}$, Nakamura A, Masaki $\mathrm{T}$, et al. A prospective comparison between multidetector-row computed tomography and magnetic resonance imaging in the preoperative evaluation of rectal carcinoma. Am J Surg 2003; 185:556-559.

16. Kulinna C, Scheidler J, Strauss T, et al. Local staging of rectal cancer: assessment with double-contrast multi-slice computed tomography and transrectal ultrasound. J Comput Assist Tomogr 2004; 28:123-130.

17. Wolberink S, Beets-Tan R, de Haas-Kock D, et al. Conventional CT for the prediction of an involved circumferential resection margin in primary rectal cancer. Dig Dis 2007; 25:8085.

18. Sinha R, Verma R, Rajesh A, et al. Diagnostic value of multi-detector row CT in rectal cancer staging: comparison of multiplanar and axial images with histopathology. Clin Radiol 2006; 61:924-31.

19. Hanley J, McNeil B, et al. A method of comparing the areas under receiver operating characteristics curves derived from the same cases. Radiology 1983; 148:839-843.

20. Cohen J. Weighted kappa: nominal scale agreement with provision for scaled disagreement or partial credit. Psychol Bull 1968; 70:213-230.

21. Klingenbeck-Regn K, Schaller S, Flohr T, et al. Subsecond multi-slice computed tomography: basics and applications. Eur J Radiol 1999; 31:110-124.

22. Schima W. MRI of the pancreas: tumours and tumour-simulating processes. Cancer imaging 2006; 6:199-203.

23. Salerno G, Sinnatamby C, Branagan G, et al. Defining the rectum: surgically, radiologically and anatomically. Colorectal Disease 2006; 8(Suppl 3):5-9.

24. Slater A, Halligan S, Taylor S, et al. Distance between the rectal wall and mesorectal fascia measured by MRI: effect of rectal distention and implications for preoperative prediction of a tumour-free circumferential resection margin. Clin Radiol 2006; 61:65-70.

25. Vliegen $R$, Beets $G$, von Meyenfeldt $M$, et al. Rectal cancer--MR imaging in local staging: is gadolinium-based contrast material helpful? Radiology 2005; 234:179-88.

26. Peschaud F, Cuenod CA, Benoist $S$, et al. Accuracy of magnetic resonance imaging in rectal cancer depends on location of the tumor. Dis Colon Rectum 2005; 48:1603-1609. 

The accuracy of MRI in predicting mesorectal fascia invasion after neo-adjuvant chemoradiation for locally advanced rectal cancer

RF Vliegen, GL Beets, G Lammering, RC Dresen, HJ Rutten, AG Kessels, Tk Oei, AP de Bruïne, JM van Engelshoven, RG Beets-Tan.

Radiology 2008; 246:454-62. 


\section{Abstract}

Purpose: To retrospectively assess the sensitivity and specificity of postchemoradiation MRI for predicting tumor invasion of the mesorectal fascia (MRF) in locally advanced primary rectal cancer, using histology/ surgery as the reference standard, and to determine morphologic MR criteria for invasion of the MRF.

Patients and methods: Two observers independently scored the postchemoradiation MR images of 64 rectal cancer patients ( 38 men, mean age of 60 years; 26 women, mean age 64 years) for tumor invasion of the MRF with a confidence level scoring system defined by subjective criteria. In a subsequent consensus reading session, morphologic MR criteria for invasion were defined by comparing morphologic changes with histologic findings. These criteria were evaluated and compared with the subjective criteria by comparing areas under the receiver operating characteristic curves (AUCs).

Results: AUCS of postchemoradiation MRI for predicting MRF tumor invasion were 0.81 and 0.82 for observer 1 and 2 .

The following four types of morphologic tissue patterns at MR imaging were associated with whether or not MRF invasion was present at histologic examination: a) development of fat pad larger than $2 \mathrm{~mm}$ (seen in no quadrants with and in four quadrants without invasion, b) development or persistence of spiculations (seen in no quadrants with and in 22 quadrants without invasion), c) development of diffuse hypointense "fibrotic" tissue (seen in 21 quadrants with and in 32 quadrants without invasion) and d) persistence of diffuse iso/hyper-intense tissue (seen in 19 quadrants with and in two quadrants without invasion).

AUC of postchemoradiation MRI for predicting MRF invasion on the basis of morphologic criteria was 0.80 . There was no statistical difference between the performance of subjective and morphologic criteria $(p=0.73-0.76)$.

Conclusion: Postchemoradiation MR imaging findings have moderate accuracy for predicting tumor invasion of the MRF related to the limitation in differentiating between diffuse "fibrotic" tissue with and without small tumor foci. Specific other types of morphologic patterns at MRI can highly predict a tumor-free or invaded MRF. 


\section{Introduction}

Locally advanced rectal cancer requires an extensive surgical resection to remove the tumor with a clear margin $(1,2)$. The rationale of neoadjuvant therapy is to downstage and downsize the tumor in order to improve resectability and to obtain better local control $(3,4)$. With a long course of radiotherapy (45-52 Gy) without chemotherapy some tumor downsizing was seen but complete pathological remissions(CR) were uncommon $(<10 \%)(5,6)$. In patients who responded, the initial tumor area was replaced by fibrotic scar tissue that often contained residual tumor nests (7). In order to achieve a complete eradication of tumor, most surgeons favoured a resection of the complete area of initial tumor, more or less regardless of the amount of response.

With more effective modern neo-adjuvant chemoradiation regimes, complete remission rates up to $38 \%$ have questioned the aggressive surgical approach to resect the complete area of the initial tumor $(8,9,10)$. An important issue of current surgical debate is whether or not it is safe to perform a less extensive resection in a patient with a locally advanced tumor that responded well to chemoradiation $(9,10)$.

Total mesorectal excision (TME) is now accepted as the standard surgical technique for the majority of rectal cancers (11). In this technique a distinct anatomical compartment called the mesorectum, containing the rectum and mesorectal fat, is removed by sharp dissection along the mesorectal fascia (MRF). Tumors that have invaded or come very close to this MRF have a higher risk for local recurrence after TME surgery, and should be considered as locally advanced (12).

MRI has repeatedly been shown to be the most accurate modality for the prediction of MRF tumor invasion, and could be an important tool in selecting patients for different types of neo-adjuvant treatment regimes according to the risk for local recurrence $(13,14,15)$. The role of MRI after neoadjuvant treatment in defining the optimal surgical resection planes has not been well studied.

Thus, the purpose of our study was to retrospectively assess the sensitivity and specificity of postchemoradiation MRI for predicting tumor invasion of the mesorectal fascia (MRF) in locally advanced primary rectal cancer, using histology/ surgery as the reference standard, and to determine morphologic MR criteria for invasion of the MRF. 


\section{Materials and methods}

\section{Patients}

The Ethics Committee of the Universtity Hospital Maastricht approved our retrospective study and waived informed consent. The records of patients with locally advanced primary rectal cancer treated at the University Hospital Maastricht and the Catharina hospital in Eindhoven between 1998 and 2006 were retrospectively studied. Locally advanced primary cancer was defined as invasion of surrounding organs or (near)invasion of the mesorectal fascia at MR imaging. Only patients who received a long course of radiation therapy or chemoradiation therapy and who subsequently underwent surgical resection were included. Further requirements for inclusion were the availability of adequate pre- and post chemoradiation MR images and detailed surgical and histologic examination reports. Overall, of a total of 390 patients with locally advanced rectal cancer, 64 patients met these inclusion criteria and formed the population of this study. The main reason for exclusion was the absence or unavailability of both pre- and post chemoradiation MR images. MR imaging was not a routine investigation in the earlier time period. There were 38 men (mean age 60 years, range 15-82) and 26 women (mean age 64 years, range 45-81). According to the initial staging MR imaging examination, eight of 64 patients had advanced T3 tumors with a tumor close to the MRF (defined as a distance of $\leq 2 \mathrm{~mm}$ or tissue strands into the mesorectal fascia), 31 of 64 patients had T3 tumors with MRF invasion and 25 of 64 patients had T4 tumors (organ invasion).

\section{Neo-adjuvant therapy}

Fifty of 64 patients received neo-adjuvant radiation therapy (50.4 Gy given in 1.8 Gy fractions in 6 weeks) together with one of the following chemotherapy regimes: (a) continuous infusion of 5 -fluorouracil (5-FU) during the radiation course $(225 \mathrm{mg} / \mathrm{sqm} / \mathrm{d})(\mathrm{n}=5)$, (b) a bolus of $5-\mathrm{FU}(350 \mathrm{mg} / \mathrm{sqm})$ plus leucovorin $(20 \mathrm{mg} / \mathrm{sqm})$ in irradiation week 1 and $5(n=14)$, (c) continuous capecitabine (two doses of $825 \mathrm{mg} / \mathrm{sqm} / \mathrm{d}$ ) during the radiation course with $(n=15)$ or without $(n=14)$ oxaliplatin $(50 \mathrm{mg} / \mathrm{sqm} /$ week) on the first day of each week, (d) capecitabine on day 1-14 and 22-35 (2x1000mg/sqm/d) combined with oxaliplatin ( $85 \mathrm{mg} / \mathrm{sqm}$ ) on day 1 and $22(\mathrm{n}=2)$. The remaining 14 patients underwent a long course of radiation therapy only, $50.4 \mathrm{~Gy}$ in $1.8 \mathrm{~Gy}$ fractions in 6 weeks. 


\section{Surgery}

Six of 64 patients underwent a standard total mesorectal excision (TME) and 4 of 64 patients a standard abdomino-perineal resection. Fifty-four of 64 patients received a more extensive resection outside the MRF with or without sphincter preservation including complete or partial resection of pelvic organ(s) in 35 of the 54 patients.

\section{MRI-technique}

MR imaging was performed on a 1.5 Tesla system (Gyroscan, Powertrack 6000 NT, Philips Medical Systems, The Netherlands) using a phased array coil (quadrature phased array spine coil, cardiac or phased array body coil). The standard protocol in both institutions consisted of T2-weighted sequences without the use of contrast enhanced sequences, fat suppression, rectal contrast or bowel relaxation. The following T2-weighted sequences were applied: turbo(fast) spin echo, TR/TE 1288-3427/150 msec, 3-4 mm slice thickness, $0.3-0.8 \mathrm{~mm}$ gap, 3-8 signal averages, $168-175 \times 240-256$ matrix, 20-32 cm FOV. The images were obtaine in 3 orientations: sagittal, coronal and axial. The latter two orientations were angled exactly perpendicular to the long axis of the tumor. All MR images were viewed on a workstation.

The median time between the initial MRI and the start of chemoradiation was 28 days (range 1-93 days), between the end of chemoradiation and the second MRI 34 days (range 1-132 days) and between postchemoradiation MRI and surgery 24 days (range 1-72 days).

\section{MR image evaluation}

The MR images were read by two observers (R.G.B.T., R.F.A.V.). Observer 1 was a dedicated pelvic MR radiologist with 12 years of experience in reading pelvic MRI. Observer 2 was a general radiologist with 8 years experience in reading MR images.

Both observers, while blinded to each other's findings and to the histologic results, independently scored the postchemoradiation MR images for tumor invasion of the MRF by using a confidence level scoring system. The following confidence level scores were used: a score of 1 indicated that tumor invasion of the MRF was definitely absent; A score of 2 , that invasion was probably absent; a score of 3 , that invasion was possibly present; a score of 4 , that invasion was probably present; and a score of 5 , that invasion was definitely present. Prechemoradiation MR examinations were available during the assessment and were used for the interpretation of postchemoradiation MR images by both observers. For the definition of these confidence level scores, both observers were free to use subjective MR criteria according to their 
individual experience with reading postchemoradiation MRI. We predefined only both ends of the confidence level spectrum. We considered the development of a fat pad larger than $2 \mathrm{~mm}$ between a residual (tumor) mass and the MRF as a definitive sign of absence of tumor invasion at postchemoradiation MR imaging [16]. At the other end, the presence of an infiltrating mass into or beyond the MRF was considered as a definitive sign of tumor invasion. The MRF was defined as the fine linear stucture enveloping the mesorectal compartment harbouring the rectum and perirectal fat, hypo-intense on T2-weighted images.

In a subsequent consensus reading session, both observers described tumor morphologic changes on the combined pre- and postchemoradiation MRI images to define morphologic postchemoradiation MR criteria. This assessment was performed (with the previous reading results and surgical and histological results at hand) in four individual quadrants of the MRF (anterior, presacral, left- and right lateral quadrants) in each patient and only at quadrants which were initially threatened or invaded by the tumor.

\section{Histology/ surgery-reference standard}

Histological evaluation of the surgical specimens were performed according to the method of Quirke (17) by two specialized pathologists with 17 years and 15 years of experience in gastro-enterologic pathology (A.d.B.). The pathological reports stated T-stage and the shortest distance between tumor and the radial resection plane(s) and resected pelvic organ(s). In patients who underwent a multivisceral resection, the topographical orientation of the residual tumor relative to the MRF was reconstructed by means of the interpretation of pathological reports in combination with detailed surgical reports and pre- and postchemoradiation MR imaging studies. In eight of 64 patients, the pathology report did not contain sufficient detailed information to enable reconstruction of the relation of the tumor to the mesorectal fascia. In these patients, histologic findings were reviewed.

\section{Statistical analysis}

The MR imaging findings were compared with MRF tumor invasion at histologic examination and surgery, which served as the reference standard. We defined MRF invasion as a distance of $\leq 2 \mathrm{~mm}$ between tumor tissue and MRF at histology (16). Failures of MRI to predict MRF invasion were reviewed by one author with 6 years of experience (R.F.A.V.) in reading rectal MRI in order to determine the cause of the failure. The interobserver agreement was calculated by using linear weighted kappa statistics based on the scored confidence levels (18).

The morphologic tissue patterns identified at MR imaging on the quadrant level were ranked according to the rate of tumor invasion at histologic 
examination, and ranking categories for predicting MRF tumor invasion were assigned to these tissue changes(R.F.V.). The value of these morphologic MR criteria for predicting MRF tumor invasion was analyzed on a per-patient level in the entire study population. When two ore more patterns were visualized in one patient, the per- patient validation was based on the most aggressive pattern of invasiveness. Receiver operating characteristic curves (ROC curves) and areas under these curves (AUC) were calculated with the confidence level scores and assigned ranking categories. The difference in performance was analyzed by comparing the corresponding AUC derived in the same set of patients and by calculating a critical ratio $z$ according to the method of Hanley and McNeil (19). A $p$ value of less than 0.05 was considered to indicate a statistically significant difference.

The confidence level scores were dichotomized for the calculation of sensitivity, specificity, positive predictive value (PPV) and negative predictive value (NPV). The cutoff level for the analysis of subjective criteria was set between confidence level scores 1 and 2 (considered to indicate no invasion) and confidence level scores 3-5 (considered to indicate tumor invasion of the MRF). For the analysis of morphologic MR criteria different cut-offs were set (as described in Results).

Statistical analysis was performed by using SPSS ${ }^{\circledR}$ software, release 11.5 (Chicago, III).

\section{Results}

The results of the analysis of postchemoradiation MR imaging versus histologic findings showed substantial overstaging of tumor invasion of the MRF at MR imaging, which occurred in $23(36 \%)$ of the 64 patients for observer 1 and in $14(22 \%)$ for observer 2 (table 1$)$. In the majority of overstaged patients (20 of 23 patients for observer 1 and 11 of 14 patients for observer 2), diffuse hypointense tissue infiltration into the MRF was noticed at MR imaging that corresponded to sterilized areas of fibrosis at histologic examination. The sensitivity for the prediction of tumor invasion of the MRF was $100 \%$ for both observers, but the specificity was only $32 \%$ for observer 1 and $59 \%$ for observer 2 (table 2).

The interobserver agreement was kappa $=0.38 \quad(95 \%$ confidence interval: 0.14-0.62). 
Table 1. Prediction of invasion of MRF with subjective criteria at postchemoradiation MR imaging by observer 1 and 2 compared with histologic findings.

\begin{tabular}{lrr}
\hline $\begin{array}{l}\text { Observer and } \\
\text { prediction at MR }\end{array}$ & $\begin{array}{c}\text { Histologic findings } \\
\text { Invasion }\end{array}$ & No invasion \\
\hline Observer 1 & & 23 \\
MR: invasion & 30 & 11 \\
$\quad$ no invasion & 0 & 14 \\
Observer 2 & 30 & 20 \\
MR: invasion & 0 & \\
$\quad$ no invasion & &
\end{tabular}

Note.- Data represent numbers of patients. Cutoff level for the definition of tumor invasion was taken between confidence level scores 1 and 2 (indicating no tumor invasion) and 3-5 (indicating tumor invasion of the MRF).

Table 2. AUC, Sensitivity, Specificity, PPV and NPV of postchemoradiation MR imaging for predicting of tumor invasion.

\begin{tabular}{lrlrl}
\hline Parameters & \multicolumn{2}{c}{ Observer 1 } & \multicolumn{2}{c}{ Observer 2 } \\
\hline AUC* & 0.81 & $(0.71-0.92)$ & 0.82 & $(0.71-0.92)$ \\
Sensitivity (\%) & 100 & $(30 / 30)$ & 100 & $(30 / 30)$ \\
Specificity (\%) & 32 & $(11 / 34)$ & 59 & $(20 / 34)$ \\
PPV (\%) & 57 & $(30 / 53)$ & 68 & $(30 / 44)$ \\
NPV (\%) & 100 & $(11 / 11)$ & 100 & $(20 / 20)$
\end{tabular}

Note.-Unless otherwise specified, numbers in parentheses are raw data.

* Data in parenthesis are $95 \%$ confidence intervals.

$\mathrm{AUC}=$ area under the ROC curve. $\mathrm{PPV}=$ positive predictive value. $\mathrm{NPV}=$ negative predictive value.

\section{Morphological tissue patterns}

Initial tumor threatening or invasion of the MRF at MR imaging was observed in 112 of a total of 256 quadrants of the MRF in 64 patients. The reference standard could not be determined based on all available information in 12/ 112 quadrants, and these quadrants were therefore excluded from the analysis; a total of 100 quadrants remained for which a surgical/ histological validation was possible. We identified the following four types of morphologic tissue patterns in the combined pre- and postchemoradiation MR imaging studies: pattern A was characterized by development of a fat pad larger than $2 \mathrm{~mm}$ between the residual tumor mass and the MRF; pattern $B$, by development or persistence of spiculations invading the MRF (figures 1,2); pattern C, by development of diffuse hypointense "fibrotic" tissue infiltrating the MRF at the initial tumor site (figure 3); and pattern $D$, by persistence of diffuse iso-/hyperintense tissue infiltrating the MRF (figure 4). 


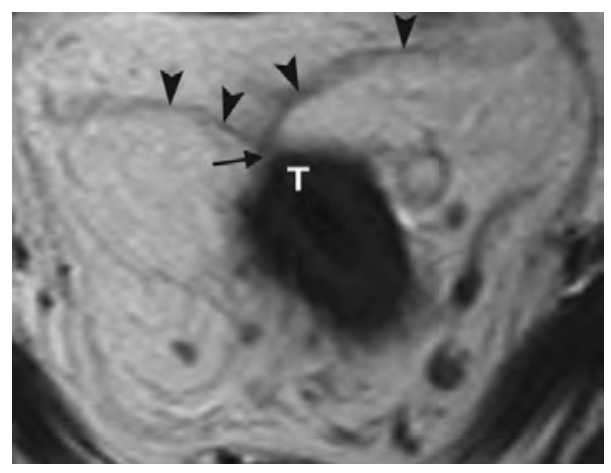

1a

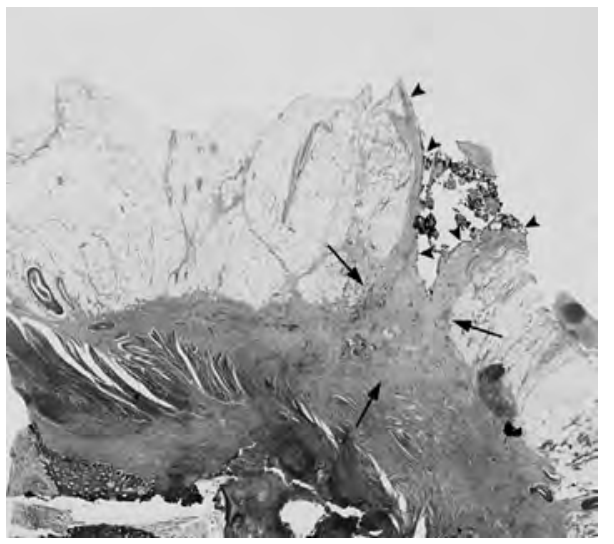

1c

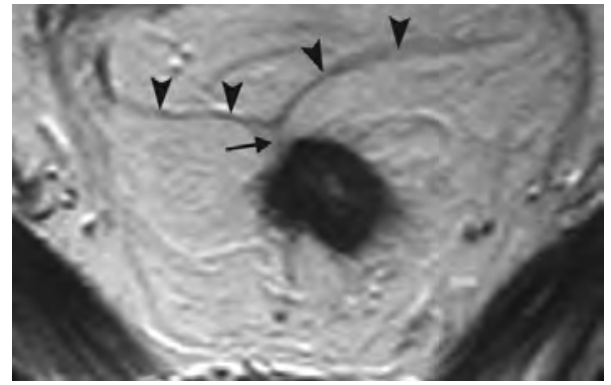

1b

Fig. 1a-c. Spiculations at pre- and post chemoradiation MR imaging correlated with a tumor-free MRF. (a,b) Transverse T2-weighted fast spin echo MR image (3427/150). (a) Prechemoradiation image shows spiculations (arrow) that reach a minimal thickened and retracted MRF (arrowheads). T=tumor. (b) Postchemoradiation image shows that these spiculations (arrow) remain unchanged; some tumor shrinkage can be noticed. Arrowheads=MRF. (c) Corresponding histologic slice (original magnification 2.5x; haematoxylin \& eosine stain) shows fibrotic spiculations (arrows) (without residual tumor tissue) in the perirectal fat that project into the circumferential resection margin (arrowheads).

Comparison of these MR imaging patterns with the reference standard histologic findings showed an increasing frequency of tumor invasion of the MRF from pattern A to pattern D (table 3). Morphologic MR criteria for the prediction of MRF tumor invasion were defined on the basis of the analysis of tissue findings and were ranked as follows: $A$ rank of 1 indicated definitely no MRF tumor invasion and included patterns $A$ and $B$, a rank of 2 indicated possible invasion and included pattern $C$, and a rank of 3 indicated probable invasion and included pattern D.

Regarding the performance of these morphologic MR criteria (table 4, figure 5) for the prediction of MRF tumor invasion at a per-patient level in the entire study population, there was overstaging of disease in $34 \%$ (22 of 64 ) of patients and understaging of disease in $0 \%$ (zero of 64 ) of patients when a cutoff level was taken between ranking category 1 (as indicating no invasion) and categories 2 and 3 (as indicating MRF invasion). A cutoff level between ranking category 1 and 2 (as indicating no invasion) and category 3 (as indicating MRF invasion) resulted in understaging of disease in $25 \%$ (16 of 64) of patients and overstaging of disease in 3\% (two of 64) of patients. 
Table 3. Morphologic tissue patterns identified in combined pre- and postchemoradiation MR imaging studies versus tumor invasion of MRF at histologic examination.

\section{Histologic findings \\ Tumor invasion No tumor invasion}

Tissue changes at MR imaging

\begin{tabular}{lrr}
\hline Pattern A: fat pad >2mm & 0 & 4 \\
Pattern B: spiculations & 0 & 22 \\
Pattern C: diffuse hypointense "fibrotic" infiltration & 21 & 32 \\
Pattern D: diffuse iso-/hyperintense infiltration & 19 & 2
\end{tabular}

Note.-Data are numbers of specific morphological patterns identified at individual quadrants of MRF in entire group of 64 patients. For further clarification of tissue change patterns, see Results section.

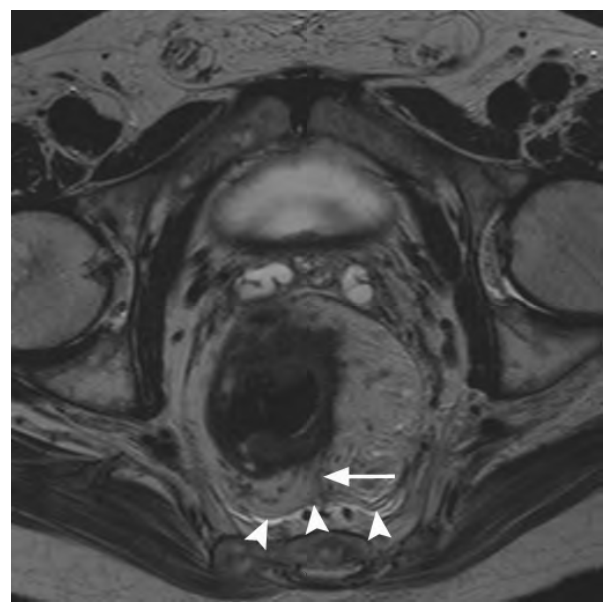

$2 \mathbf{a}$

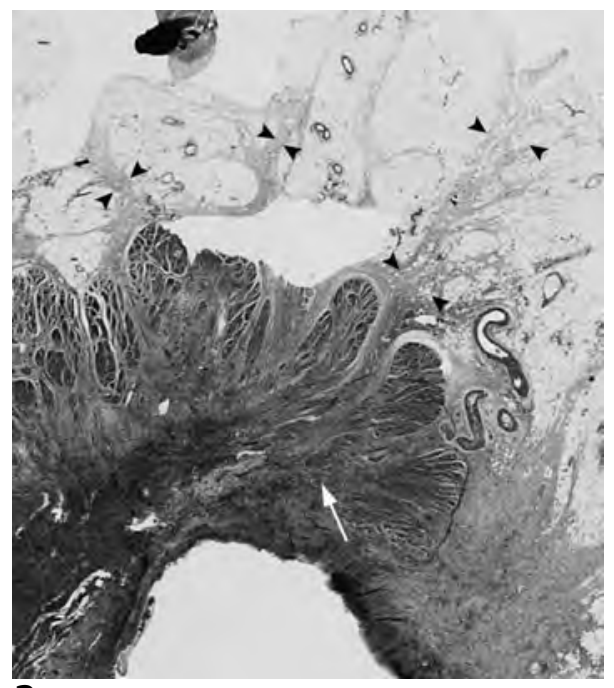

2c

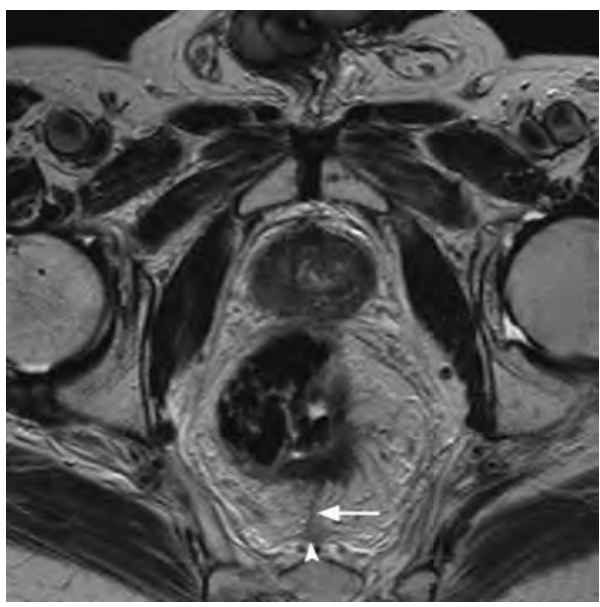

2b

Fig. 2a-c. Spiculations at pre- and postchemoradiation MR imaging were associated with a tumor-free MRF. $(a, b)$ Transverse T2-weighted fast spin echo MR images (3427/150). (a) Prechemoradiation image showins spiculations (arrow) projecting into the MRF (arrowheads). (b) Postchemoradiation image shows that these spiculations (arrow) remain unchanged. Arrowhead=MRF. (c) Corresponding histologic slice (magnification $5 x$; H\&E stain) shows residual tumor limited to the rectal wall (arrow) and fibrotic spiculations (arrowheads) without tumor tissue projecting into the circumferential resection margin. 


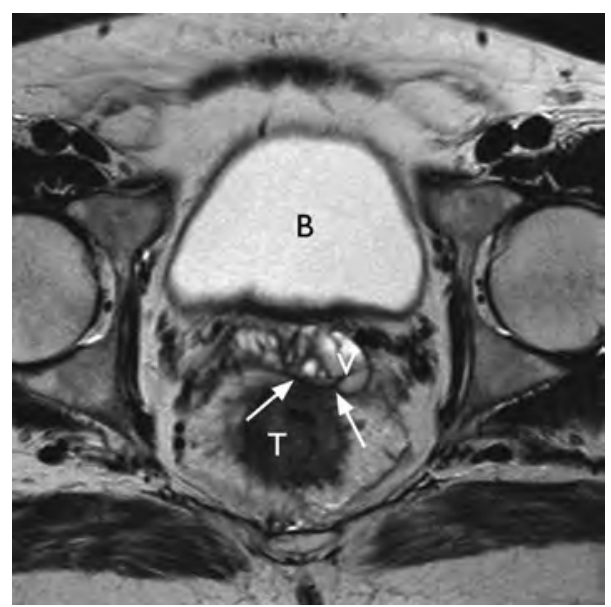

3a

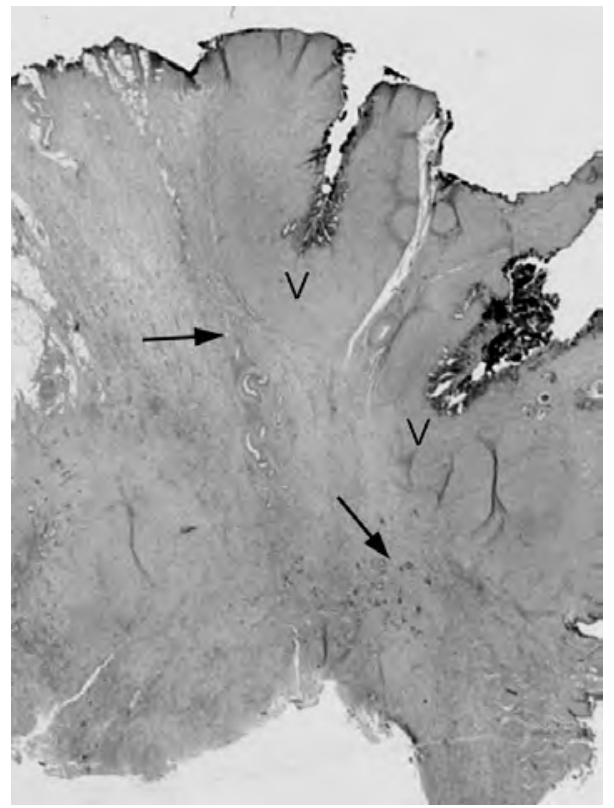

3c

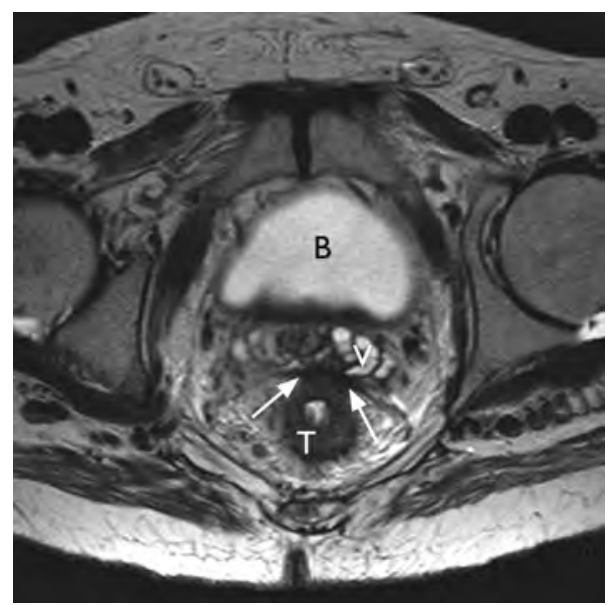

3b

Fig. 3a-c. Diffuse hypointense tissue infiltration at postchemoradiation MR imaging suggestive of fibrosis can be associated with MRF tumor invasion caused by residual tumor nests within fibrosis. $\mathrm{B}=$ bladder, $\mathrm{T}=$ tumor, $V=$ seminal vesicle. $(a, b)$ Transverse $T 2$-weighted fast spin echo MR image (3427/150). (a) Prechemoradiation image shows diffuse isointense invasion of the anterior MRF (arrows) and left seminal vesicle. (b) Postchemoradiation image shows that this type of invasion has changed into diffuse hypointense tissue infiltration (arrows), suggesting diffuse fibrosis. (c) Corresponding histologic slice (magnification 2.5x; H\&E stain) shows a diffuse fibrotic reaction (arrows) that reaches the seminal vesicle and contains small residual tumor nests. These small tumor nests cannot be seen at MR imaging; therefore, it is impossible to differentiate fibrosis with from that without small tumor nests at MR imaging. 
Table 4. Performance of morphologic MR imaging criteria for prediction of tumor invasion of MRF at per-patient level with different cutoff levels.

\begin{tabular}{lrrr}
\hline Parameters & Cutoff level 1* & \multicolumn{2}{c}{ Cutoff level 2** } \\
\hline Sensitivity (\%) & $100(30 / 30)$ & $47(14 / 30)$ \\
Specificity (\%) & $35(12 / 34)$ & $94(32 / 34)$ \\
PPV (\%) & $58(30 / 52)$ & $88(14 / 16)$ \\
NPV (\%) & $100(12 / 12)$ & $67(32 / 48)$
\end{tabular}

Note.-Unless otherwise specified, numbers in parentheses are raw data. The AUC for the overall performance of morphologic MR imaging criteria for predicting tumor invasion of the MRF was 0.80 (95\% confidence interval: $0.69-0.90)$

* Cutoff level set between ranking category 1 (no invasion) and category 2 and 3 (tumor invasion of the MRF).

** Cutoff level set between ranking category 1 and 2 (no invasion) and category 3 (tumor invasion of the MRF).

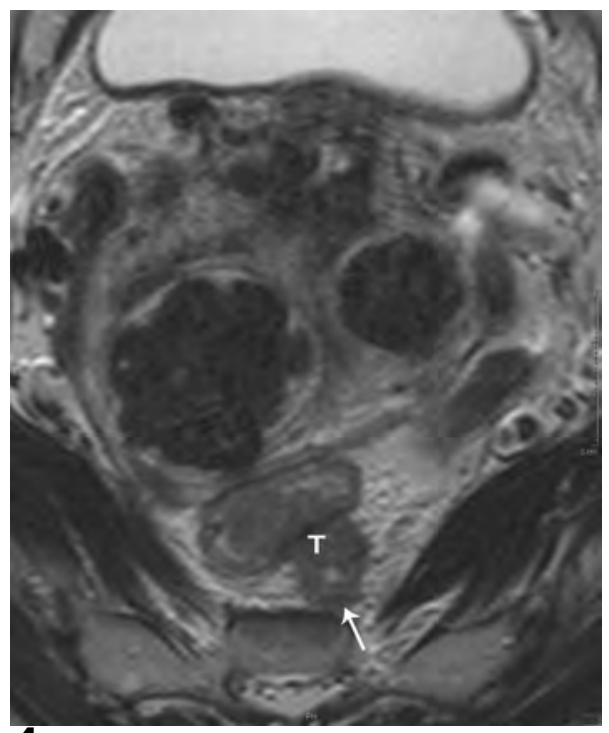

$4 \mathbf{a}$

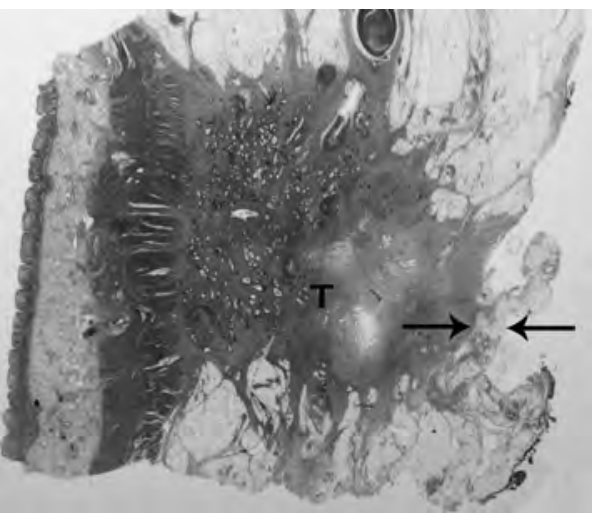

4c

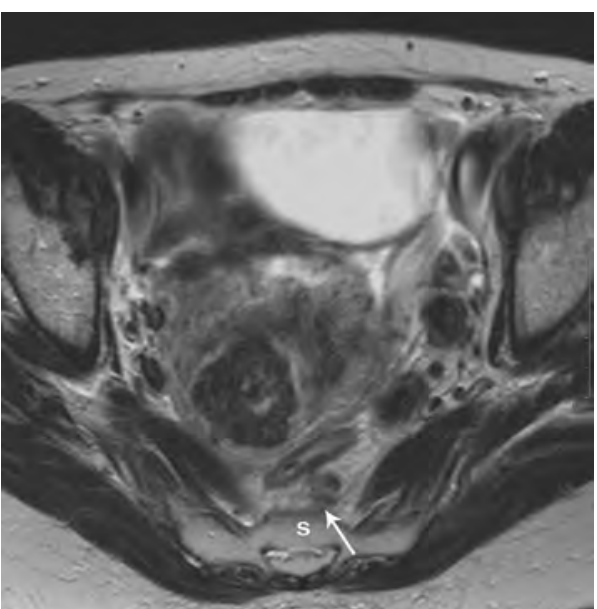

4b

Fig. 4a-c. Diffuse hyperintense tissue infiltration (morphologic tissue pattern D) associated with tumoral invasion. $(a, b)$ Transverse T2-weighted fast spin echo MR images (3427/150). (a) Prechemoradiation image shows hyperintense (compared with muscle) tissue invasion of the presacral MRF (arrow). T=tumor. (b) Postchemoradiation image shows that diffuse hyperintense infiltration of the MRF (arrow) is still present. $\mathrm{S}=$ sacrum. (c) Corresponding histologic slice (magnification $5 x$; H\&E stain) shows gross residual tumor $(T)$ near the circumferential resection margin, with only a small tumor-free margin (between arrows). This was considered as proof of tumor invasion of the MRF because the surgeon performed a wide extramesorectal surgical excision posteriorly. 
Fig. 5. Flowchart of patients inclusion and results of postchemoradiation MR imaging for prediction of tumor invasion of the MRF by application of MR imaging criteria.

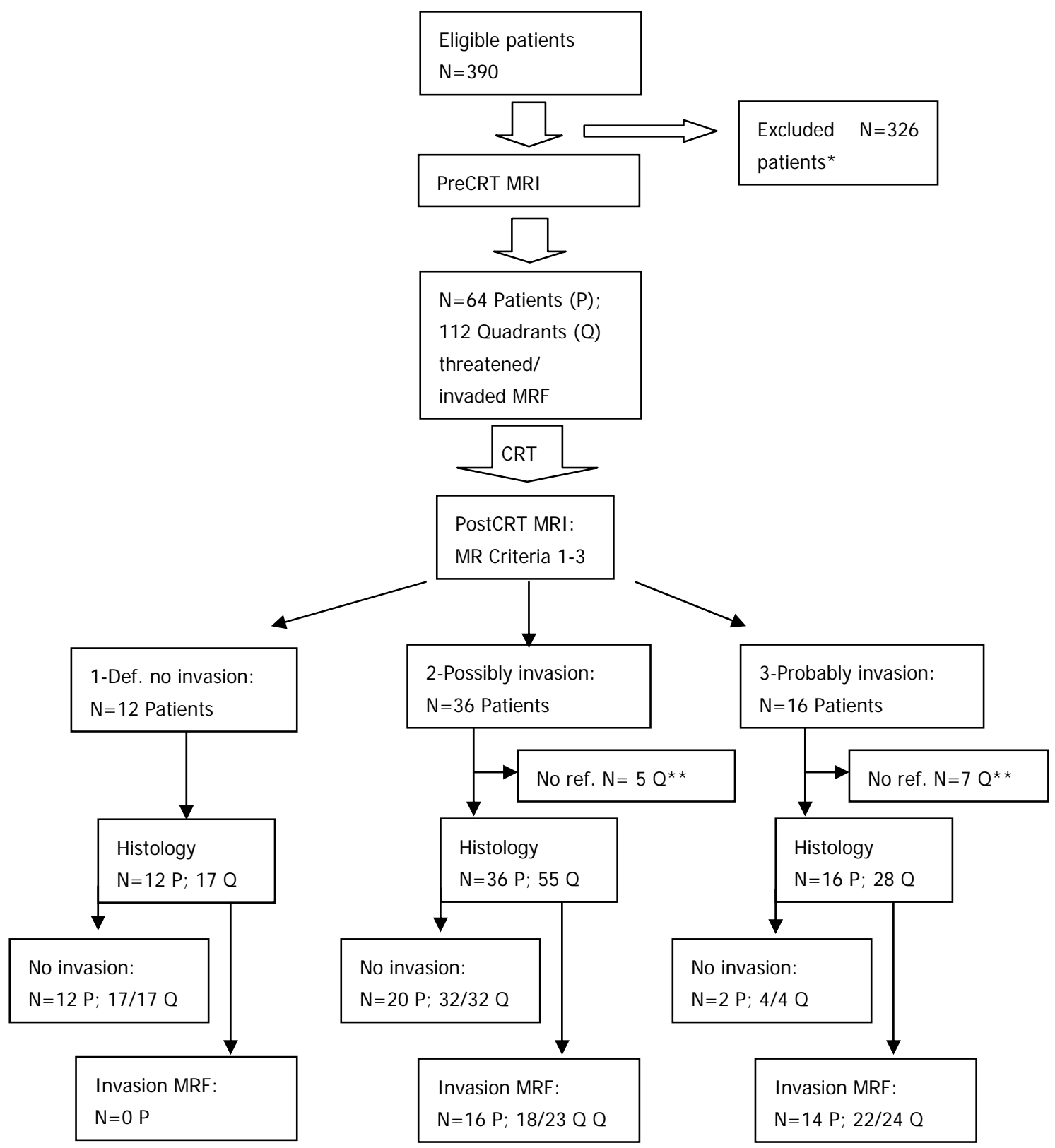

Note.- Prediction of invasion of the MRF at a per-patient level was determined by the most aggressive MR imaging pattern observed. ${ }^{*}=$ See patient inclusion criteria in Materials and Methods section, $* *=$ individual quadrants without reference standard in patients with histologically proven tumor invasion of the MRF at other quadrant(s). Def.=definitely, PreCRT=prechemoradiation, ref. $=$ reference standard. 
There was no statististical difference between the performance of subjective criteria used by the observers and morphologic MR criteria for the prediction of MRF invasion on postchemoradiation MRI $(p=0.73-0.76)$.

\section{Discussion}

Our study evaluated the performance of postchemoradiation MRI for the prediction of MRF tumor invasion. We found a relatively high AUC (0.81-0.82), high sensitivity and NPV (both $100 \%$ ) for both observers. However, our results showed only a moderate specificity (32-59\%) and PPV (57-68\%).

The main difficulty of postchemoradiation MRI in the assessment of MRF tumor invasion is the assessment of diffuse hypo-intense "fibrotic" tissue in the initial tumor area, a feature seen in more than $50 \%$ of patients. In one of three quadrants, this "fibrotic" tissue at MR imaging showed tumor infiltration at histologic examination. Residual tumor within these fibrotic areas is often confined to small tumor nests that are beyond the detection level of MRI $(7,20)$. It is therefore virtually impossible to differentiate this from completely sterilized areas of fibrosis.

Although no other studies have addressed the question of the prediction of MRF tumor invasion with MR imaging after long courses of chemoradiation, there are some reports on the prediction of T stage at MRI after chemoradiation therapy (21-23). These have reported accuracies of only $47-53 \%$, confirming the limited ability of postchemoradiation MR imaging to help differentiate between viable tumor, fibrotic tissue, and inflammatory reaction.

Our experienced observers were generally aware of the limitation of MR imaging in depicting residual tumor disease in diffuse hypointense "fibrotic" areas and set their cutoff levels such as to minimize the number of false negative findings, at the expense of many false positive findings. From a clinical point of view, overstaging of disease is much more acceptable than understaging, as understaging may lead to incomplete tumor resection and an unacceptable high risk of local tumor recurrence $(1,2,24)$.

The moderate interobserver agreement $(\mathrm{kappa}=0.38)$ between both experienced observers implies substantial difficulty interpreting postchemoradiation MR images, and this may be a reflection of the lack of uniform postchemoradiation MR imaging criteria for tumor invasion of the MRF. The application of uniform MR criteria on the basis of our analysis of morphologic tissue changes did not improve the staging results compared with the subjective assessment of both observers $(p=0.73-0.76)$. We still encountered substantial overstaging in 34\% (22 of 64) of patients when the cutoff level was set so as to accept no understaging. On the other hand, dramatic understaging occurred in $25 \%$ (16 of 64 ) of patients when a less 
conservative cutoff level was chosen by considering cases of diffuse "fibrotic" tissue infiltration as negative for MRF tumor invasion.

Despite the problems of MR imaging in the interpretation of postradiotherapy fibrosis, some potentially useful specific morphologic patterns were identified. The presence of diffuse iso-/hyperintense tissue infiltration of the MRF at MR imaging was associated with tumor invasion at histologic examination in $90 \%$ (19 of 21 ) of the quadrants in which this pattern was seen. On the other hand, when spiculations had developed at postchemoradiation MR imaging or were seen in the combined pre- and postchemoradiation MR imaging studies, this was always associated with a tumor free MRF at histologic examination (22 of 22 quadrants). We are aware that in some of these patients, the initial tumor invasion of the MRF before chemoradiation therapy was debatable, as in some patients the tumor was close to the fascia rather than invading it $(13,25)$.

When considering the results of our study, one must keep in mind that the design of the study had some limitations. First, although the reference standard of this study was based on the combined analysis of detailed standardized pathological reports, detailed surgical reports, MR imaging studies and, in some patients, reviewing histologic results, inaccuracies might have been introduced because of the retrospective nature of this study. Second, a selection bias might have been introduced because of the inclusion of nonconsecutive patients and the exclusion of MR imaging features seen in quadrants for which no reference standard could be reconstructed. This occurred, however, in only a minority of quadrants; therefore, we believe this does not substantially influence our main findings. The third limitation was the time span between the postchemoradiation MR imaging examinations and the resection. In this period, with a median of 24 days, a further regression of the tumor is likely to occur, and theoretically, this could account for some of the overstaging errors that mainly occurred in the interpretation of areas of fibrosis (26). This should be kept in mind when interpreting the results.

Furthermore, the MR protocol could have been a matter of debate. After it had been shown in a previous study that static Gadolinium enhanced T1-weighted sequences had no additional value in assessing MRF invasion in nonirradiated rectal cancer, these sequences were omitted from the standard MR protocol in our department (25). The use of dynamic contrast enhanced sequences has been reported to be useful in other applications such as differentiating tumor (recurrences) from benign (postoperative) tissue by measuring time-intensity curves or perfusion indexes $(27,28)$. However, it remains questionable whether these techniques would offer any help for the main problem in our study: the detection of small areas of tumor within poorly vascularized fibrotic tissue. Positron emission tomography (PET) suffers from the same limitation, as illustrated in a recent publication that reported no increased tracer uptake in the majority of responding tumors with small residual 
areas of tumor disease (29). Additionally, the increased uptake of 18-FDG by non tumorous inflammatory tissue after neoadjuvant therapy could lead to interpretational errors.

If imaging of small amounts of viable tumor is ever to be successful, we believe it will be through advances in the field of molecular imaging with PET or MRI. When considering the above described limitations of interpreting postchemoradiation MR imaging studies, one could question their usefulness. Many surgeons will choose to resect the complete initial tumor area, more or less irrespective of the amount of response. At present it is unclear if and when it is safe to perform more conservative resections on the basis of imaging findings of the response, and so far the present standard of care does not take into consideration the postchemoradiation MR findings. However, with higher response rates and better imaging methods, surgeons will be forced to rethink this strategy. At present, even with its limitations, we still perform a postchemoradiation MR imaging. Its results help tell the surgeon what to expect, and occasionally for example, in frail patients with a good response, a more conservative resection can be performed. A resection along the MRF (Total mesorectal excision surgery) seems to be justified when "fibrotic" spiculations are identified at postchemoradiation MR imaging. It is clear that when diffuse iso-/hyperintense "tumor" infiltration is seen at MR imaging, this site requires whole area resection. Maybe even of more importance is the fact that MR imaging may alert the surgeon and change the resection plan in case of tumor progression after chemoradiation therapy. It is the most commonly seen pattern of diffuse hypointense "fibrotic" tissue infiltration that remains difficult to interpret. We inform the surgeon of the chances of an invaded MRF (one out of three in our population), and it is up to the surgeon to weigh the benefit of a less aggressive surgical approach against a potentially positive resection margin.

\section{Conclusion}

Postchemoradiation MR imaging has a moderate accuracy (sensitivity, $100 \%$; specificity, $32-59 \%$ ) for predicting tumor invasion of the MRF that is related to the inherent limitation of differentiating between diffuse "fibrotic" tissue harboring small tumor foci and completely sterilized areas of fibrosis. "Fibrotic" areas should therefore be considered as potentially invaded. Specific other types of morphologic tissue changes at MR imaging can highly predict a tumor-free or invaded MRF. 


\section{References}

1. Govindarajan A, Coburn N, Kiss A, et al. Population based assessment of the surgical management of locally advanced rectal cancer. J Natl Cancer Inst 2006;98:1474-1481.

2. Lehnert T, Methner M, Pollok A, et al. Multivisceral resection for locally advanced primary colon and rectal cancer: an analysis of prognostic factors in 201 patients. Ann Surg 2002; $235: 217-225$.

3. Sauer $R$, Beckers $H$, Hohenberger $W$, et al. Preoperative vs postoperative chemoradiotherapy for rectal cancer. N Engl J Med 2004;351:1731-1740.

4. Reerink O, Verschueren R, Szabo B, et al. A favourable pathological stage after neoadjuvant radiochemotherapy in patients with initially irresectable rectal cancer correlates with a favourable prognosis. Eur J Cancer 2003;39:192-195.

5. Tobin R, Mohiuddin M, Marks G. Preoperative irradiation for cancer of the rectum with extrarectal fixation. Int J Radiat Oncol Biol Phys 1991;21:1127-1132.

6. Read $T$, Olajungu $A$, Ogunbiyi $O$, et al. Neoadjuvant external beam radiation and proctectomy for adenocarcinoma of the rectum. Dis Colon Rectum 2001;44:1778-1790.

7. Dworak 0 , Keilholz L, Hoffmann A. Pathological features of rectal cancer after preoperative radiochemotherapy. Int J Colorect Dis 1997; 12:19-23.

8. Hartley A, Ho K, McConkey $\mathrm{C}$, et al. Pathological complete response following preoperative chemoradiotherapy in rectal cancer: analysis of phase II/III trials. $\mathrm{Br} J$ of Radiol 2005; 78:934-938.

9. Bonnen M, Crane C, Vauthey J, et al. Long-term results using local excision after preoperative chemoradiation among selected T3 cancer patients. Int J Radiat Oncol Biol Phys 2004;60:1098-1105.

10. Habr-Gama A, Perez R, Nadalin W, et al. Operative versus nonoperative treatment for stage 0 distal rectal cancer following chemoradiation therapy: long-term results. Ann Surg 2004;240:711-717.

11. Ulrich A, Schmidt J, Weitz J, et al. The Heidelberg results after TME. Recent Results Cancer Res 2005; 165:112-119.

12. Kapiteijn E, Marijnen C, Nagtegaal I, et al. Preoperative radiotherapy combined with total mesorectal excision for resectable rectal cancer. N Engl J Med 2001;345:638-646.

13. Beets-Tan R, Beets $G$, Vliegen $R$, et al. Accuracy of magnetic resonance imaging in prediction of tumour-free resection margin in rectal cancer surgery. The Lancet 2001; 357:497-504.

14. Burton S, Brown G, Daniels I, et al. MRI directed multidisciplinary team preoperarive treatment strategy: the way to eliminate positive circumferential margins? BJC 2006; 94:351-357.

15. Lahaye $M$, Engelen $S$, Nelemans $P$, et al. Imaging for predicting the risk factors--the circumferential resection margin and nodal disease--of local recurrence in rectal cancer: a meta-analysis. Semin Ultrasound CT MR; 2005;26:259-268.

16. Nagtegaal I, Marijnen C, Kranenbarg E, et al. Circumferential margin involvement is still an important predictor of local recurrence in rectal carcinoma: not one millimeter but two millimeters is the limit. Am J Surg Pathol 2002;26:350-357.

17. Quirke $P$, Dixon $M$. The prediction of local recurrence in rectal adenocarcinoma by histopathological examination. Int J Colorectal Dis 1988;3:127-131.

18. Cohen J. Weighted kappa: nominal scale agreement with provision for scaled disagreement or partial credit. Psychol Bull 1968;70:213-230.

19. Hanley J, McNeil B. A method of comparing the areas under receiver operating characteristic curves derived from the same cases. Radiology 1983;148:839-843.

20. Beets-Tan R, Beets G, Borstlap A, et al. Preoperative assessment of local tumor extent in advanced rectal cancer: CT or high resolution MRI. Abdom Imaging 2000;25:533-541. 
21. Kuo L, Chern M, Tsou M, et al. Interpretation of magnetic resonance imaging for locally advanced rectal carcinoma after preoperative chemoradiation therapy. Dis Colon Rectum 2005; 48:23-28.

22. Hoffmann K, Rau B, Wust $P$, et al. Restaging of locally advanced carcinoma of the rectum with MR imaging after preoperative radio-chemotherapy plus regional hyperthermia. Coloproctology 2002;24:253-261.

23. Chen $\mathrm{C}$, Lee $\mathrm{R}$, Lin $\mathrm{J}$, et al. How accurate is magnetic resonance imaging in restaging rectal cancer in patients receiving preoperative combined chemoradiotherapy? Dis Colon Rectum 2004;48:722-728.

24. Luna-Perez P, Bustos-Cholico E, Alvarado I, et al. Prognostic significance of circumferential margin involvement in rectal adenocarcinoma treated with preoperative chemoradiotherapy and low anterior resection. J Surg Oncol 2005;90:20-25.

25. Vliegen $R$, Beets $G$, von Meyenfeldt $M$, et al. Rectal cancer-MR imaging in local staging: is gadolinium-based contrast material helpful? Radiology 2005;234:179-188.

26. Moore $\mathrm{H}$, Gittleman A, Minsky B, et al. Rate of pathologic complete response with increased interval between preoperative combined modality therapy and rectal cancer resection.Dis Colon Rectum 2004;47:279-286.

27. Toricelli P, Pecchi A, Luppi G, et al. Gadolinium-enhanced MRI with dynamic evaluation in diagnosing the local recurrence of rectal cancer. Abdom Imaging 2003;28:19-27.

28. Rudisch A, Kremser C, Judmaier W, et al. Dynamic contrast-enhanced magnetic resonance imaging: a non-invasive method to evaluate significant differences between malignant and normal tissue. Eur J Radiol 2005;53:514-519.

29. Capirci C, Rubello D, Chierichetti $F$, et al. Restaging after neoadjuvant chemoradiotherapy for rectal adenocarcinoma: role of F18-FDG PET. Biomed Pharmacother 2004;58:451-457. 
How accurate can sequential FDG-PET/CT predict tumor regression from the mesorectal fascia in locally advanced rectal cancer treated with neo-adjuvant chemoradiation therapy?

RF Vliegen, RG Beets-Tan, B Vanhauten, A Driessen, M Ollers, AG Kessels, Al Arens, J Buijsen,

AA van Baardwijk, DK De Ruysscher G Lammering.

Submitted for publication. 


\section{Abstract}

Purpose: To evaluate the value of 18F-fluorodeoxyglucose Positron Emission Tomography/ Computed Tomography (PET/CT) for predicting tumor regression from the mesorectal fascia (MRF) in locally advanced rectal cancer patients treated with neo-adjuvant combined chemo- and radiotherapy (CRT).

Materials and methods: Twenty $\mathrm{T} 3-4 \mathrm{NxMx}$ rectal cancer patients with Magnetic Resonance Imaging proven tumor invasion of the MRF underwent PET/CT before and on average 6.3 weeks after CRT followed by surgery. Tumor volumes were outlined on both PET/CT's using auto-contouring software and the maximal Standard Uptake Values (SUVmax) were registered in the outlined tumor volumes. The shortest distance between the outlined tumor volume and the MRF was determined after CRT. The surgical specimen were carefully evaluated for tumor regression from the mesorectal fascia (TRmrf) and the tumor regression grade (TRG)

Results: The TRG corresponded significantly with the SUVmax changes induced by CRT (responders=TRG1-2 vs non-responders=TRG3-5: $83.0 \%$ vs $59.4 \%$ SUVmax reduction; $p=0.025)$, and showed a trend with the postCRT SUVmax (TRG1-2 vS TRG3-5: SUVmax $=3.0$ vs $5.0 ; p=0.059$ ).

However, the pathologically verified tumor regression from the MRF was not correlated with neither any of the tested SUVmax parameters (TRmrf=SUVmax $14.2 \pm 4.4$, dropping by $75.0 \pm 18.4 \%$ to $3.7 \pm 2.1$ vs no TRmrf=SUVmax $13.4 \pm 4.6$, dropping by $54.5 \pm 28.9 \%$ to $5.4 \pm 2.3$; p $>0.1$ ) nor with the shortest distance between the automatically outlined residual tumor volume and the MRF (TRmrf $=2.1 \mathrm{~mm} \pm 3$ vs no TRmrf $=1.8 \mathrm{~mm} \pm 3 ; p>0.1$ ).

Conclusion: Our data suggest that PET/CT is a poor indicator for anatomical changes in rectal tumors after CRT and therefore not accurate for prediction of tumor regression from the MRF. However PET/CT is a good indicator for pathological tumor response enabling early differentiation between responders and non responders to neoadjuvant chemoradiation 


\section{Introduction}

Locally advanced primary rectal cancer carries a poor prognosis. Aggressive multi-modality regimes have been introduced in the treatment of these unfavourable group of patients in order to improve local and distant tumor control $(1,2)$. Until now, neo-adjuvant therapy followed by extensive surgical resection of the complete initial tumor area is the treatment of choice, however this aggressive approach carries high treatment related morbidity (1-4).

The recent introduction of highly effective neo-adjuvant chemoradiation regimes offer the promise to significantly improve tumor response rates in locally advanced rectal cancer (5). These good responses with estimated complete pathological remission rates of up to $25 \%$ have questioned the aggressive surgical approach and have opened opportunities for more individualized, conservative surgical treatment strategies for locally advanced rectal cancer (5-7). An important issue in current surgical debate is whether or not it is safe to perform a less extensive resection in patients with locally advanced rectal cancer that responded well to neo-adjuvant chemoradiation therapy (6-7).

Total mesorectal excision (TME), which is the standard surgical procedure for non locally advanced rectal cancer may become an attractive option for initially locally advanced rectal tumors, for which a tumor regression from the mesorectal fascia has been identified preoperatively after neoadjuvant CRT (8). With this surgical technique a distinct anatomical compartment called the mesorectum, containing the rectum and perirectal fat, is removed by sharp dissection along the mesorectal fascia which defines the circumferential resection margin (9). This, however, requires decision tools, which provide clear and safe criteria for the prediction of the tumor regression from the mesorectal fascia. Non- invasive imaging tools could provide such criterias, however, conventional imaging methods like CT and MRI, although providing high anatomical detail information, have failed in accurately predicting tumor response after CRT due to the misinterpretation of posttherapeutic non-tumoral related effects (10-12).

The integration of 18F-fluoro-deoxy-glucose (FDG)-PET in the restaging of the rectal tumor after CRT might be able to serve as a promising decision tool for proper response evaluation, since it has recently been demonstrated to be superior to CT and MRI for the prediction of tumor response (13). A more conservative surgical approach might thus be considered for those patients, who have been defined by PET as responders. The main problem of PET, however lies in the spatial resolution limitation which is required for surgical anatomical roadmaps to plan the extent of surgical resection.

We therefore hypothesized that the use of an integrated PET/CT providing combined anatomical and metabolic information might be useful to serve as a tool to identify patients, whose tumors regressed from the mesorectal fascia after CRT, thus offering these patients a more conservative 
TME surgery. This would not only safe time and money for the hospitals, but also substantially reduce the postoperative morbidity for this group of patients, thereby improving the quality of life. To our knowledge, no studies have been reported on this subject. Therefore, the aim of our study was to evaluate the value of an integrated PET/CT scan for predicting tumor regression from the mesorectal fascia in locally advanced rectal cancer patients treated with neoadjuvant CRT.

\section{Materials and methods}

\section{Patients}

The records of a cohort of 45 consecutive patients with locally advanced rectal cancer were searched for patients who underwent sequential PET/CT imaging prior to and after the application of a combination of neo-adjuvant chemotherapy and radiotherapy at the department Maastro clinic between March 2004 and March 2005. From this group, patients with tumor invasion of the mesorectal fascia prior to chemoradiation (CRT) treatment, diagnosed with Magnetic Resonance Imaging (MRI) were selected. Further inclusion criteria were: available pre- and post PET/CT examinations, surgical resection of the tumor, available histologic specimen, available pathologic and surgical reports. In total 20 patients met the inclusion criteria and were enrolled in the present study. There were 12 men with a mean age of 63 years (range 44-78 years) and 8 women with a mean age of 63 years (range $46-85$ years). Twelve of 20 patients were diagnosed with locally advanced T3NxMx tumors with tumor invasion of the mesorectal fascia and 8 of 20 patients with locally advanced T4NxMx tumors as determined by pre-CRT MRI.

\section{Imaging and therapy}

Patients underwent PET/CT imaging on average $9 \pm 7$ days before the initiation of CRT. The CRT consisted of a long course of radiotherapy (50.4 Gy given in 28 fractions of $1.8 \mathrm{~Gy}$; one fraction a day) together with oral administration of capecitabine $(2 \times 825 \mathrm{mg} / \mathrm{sqm} / \mathrm{d})$. CRT was followed by a restaging PET/CT scan (mean elapsed time end of CRT-PET/CT: $44 \pm 10$ days, range 20-70 days) and surgery (mean elapsed time PET/CT-surgery: $20 \pm 17$ days, range 1-63 days). Four of the in total 20 patients underwent a total mesorectal excision (TME), 3 of 20 patients received abdominal-perineal resection and 13 of 20 patients underwent more extensive surgical resections outside the confines of the mesorectal compartment including complete or partial resection of pelvic organ(s) in 9 of 13patients. The decisions for the extent of surgical resections were based on the combined pre- and post CRT $\mathrm{MRI}$ and clinical findings. 


\section{PET/CT technique}

All PET/CT studies were performed on an integrated in-line PET/CT system (Biograph ${ }^{\mathrm{TM}}$ SOMATOM $^{\circledR}$ Sensation 16, Siemens, Erlangen, Germany). Patients were instructed to fast at least 6 hours before undergoing the examination. All patients had fasting blood glucose levels between 3,5 and $7,2 \mathrm{mmol} /$ liter (mean glucose level of $5.1 \mathrm{mmol} /$ liter). 18F-fluoro-2-deoxyglucose (FDG) (MDS Nordion, Liège, Belgium) was injected intravenously, followed by physiologic saline $(10 \mathrm{ml})$. The injected dose was dependent on the weight of the patient (weight $x 4+20 \mathrm{MBq}$ ) ranging from $204 \mathrm{MBq}-427 \mathrm{MBq}$ (mean $344 \mathrm{MBq}$ ) of 18F-FDG. First, the whole body (from skull to mid thich) CT data set ( $120 \mathrm{keV}, 140 \mathrm{~mA}$, pitch 1.25, $5 \mathrm{~mm}$ reconstructed slices to match PET section thickness, supine position) was acquired after a period of 45 minutes. The majority of patients (18 of 20$)$ received iv contrast $(120 \mathrm{ml}$ non-ionic iodine iv contrast, Xenetix ${ }^{\circledR}$, Guerbet, France) at a flow rate of $3 \mathrm{ml} / \mathrm{sec}$. Immediately after the CT, a PET-scan was performed covering the identical transverse field of view $(500 \mathrm{~mm})$. PET data acquisition was performed in 5 -minutes bed positions (total of seven bed positions with overlap). PET images were corrected for scatter and attenuation using a CT based attenuation map. Image reconstruction was performed using FORE (Fourier rebinning) and OSEM reconstruction (ordered subsets expectation maximization, 4 iterations, 8 subsets) with an inter-slice distance of $3.45 \mathrm{~mm}$.

\section{PET/CT data analysis}

The PET/CT data were sent to a state-of-the-art multi-modality computer platform for image review and manipulation (Wizard, Siemens, Germany).

The maximal Standard Uptake Value (SUVmax), calculated as the maximal activity concentration of FDG uptake divided by injected dose/ body weight) was used to measure the amount of FDG uptake of the tumor in a selected ROI (region of interest). The tumor volume was automatically delineated for each patient before and after CRT by using auto-contouring software (Esoft 5.0.24.5, Siemens, Germany). The mean volume of the tumor prior to chemoradiation was $53.9 \mathrm{~cm}^{3}$ (range $13.2-101.4 \mathrm{~cm}^{3}$ ) and $15.0 \mathrm{~cm}^{3}$ (range $0-40.7 \mathrm{~cm}^{3}$ ) after chemoradiation. For the determination of the background activity, a ROI (volume of $4.0 \mathrm{~cm}^{3}$ ) was manually drawn over the left gluteus muscle.

Secondly, for each patient, the shortest distance (in $\mathrm{mm}$ ) between the automatically outlined tumor volume (by using e-soft) and the mesorectal fascia was measured after chemoradiation on the fused PET/CT images by two observers, blinded for the clinical results (see figure 1). The 
mesorectal fascia was defined as the fine linear structure (isodense to muscle) surrounding the mesorectal compartment including the rectum and peri-rectal fat tissue, as seen on the fused PET/CT images.

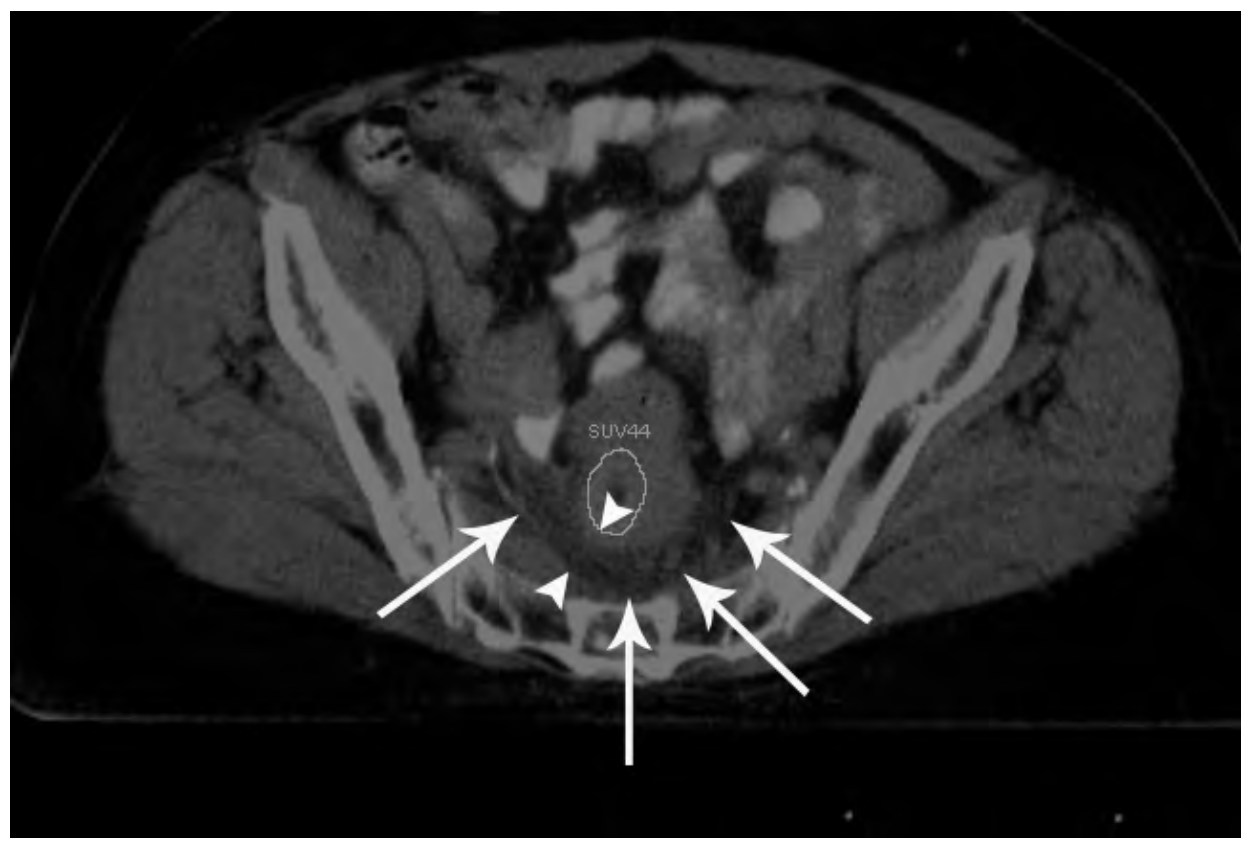

Fig. 1. Axial postCRT PET/CT image. Example of performed measurements of the shortest distance (between arrowheads) between the outlined tumor volume (encircled) by auto-contouring software and the mesorectal fascia (arrows) on a postCRT PET/CT. image The mesorectal fascia (arrows) is an anatomical structure surrounding the mesorectal compartment. This fascia serves as the anatomical resection plane in total mesorectal excision surgery and therefore defines the circumferential resection margin.

\section{Method of auto-contouring and validation in a phantom model}

The applied method of auto-contouration was based on the automated contouring of volumes exceeding a specified threshold on PET determined by the measured signal to background ratio (SBR) $(14,15)$. The applied tresholds (depending on the measured SBRs) varied between 22 and $42 \%$ (mean $31.1 \%$ ) on the examinations prior to chemoradiation and between 36 and $69 \%$ (mean $43.8 \%$ ) after the application of chemoradiation.

Phantom studies were performed to determine the relationship between the SBR and treshold of activity. For this purpose, measurements were performed using a cylindrical phantom containing six spherical inserts. The volumes of the spheres ranged from 2 to $16 \mathrm{ml}$ and contained an equal concentration of FDG (approximately $200 \mathrm{kBq} / \mathrm{ml}$ ). The background FDG concentration was varied stepwise yielding signal-to-background 
concentrations (SBRs) between approximately 2 and 12. The relationship between the thresholds of activity, defined as a percentage of maximal uptake in the spheres when the estimated volumes matched the actual volume of the spheres and the SBRs, was then determined.

SUV contouring occurred on a sub-grid scale i.e. below the PET pixel resolution of approximately $4 \mathrm{~mm}$. The geometrical accuracy of the SUV contour was verified by performing a PET/CT scan of a concentric sphere. The inner sphere (diameter) and the outer sphere were filled with FDG such that a clinically relevant source-to-background ratio was achieved. On each reconstructed slice two SUV contours were created encompassing the inner and outer sphere respectively. The distance between the two SUV contours on the centre slice (passing through the origin of the spheres) corresponded exactly with the actual distance $(5.3 \mathrm{~mm})$ between the shell of the inner sphere and the shell of the outer sphere. From these experiments we concluded that the SUV contours were accurate and reliable in a phantom model.

\section{Histologic examination}

Histologic evaluation of the surgical specimens was performed according to the method of Quirke by a team of pathologists specialized in gastro-enterologic pathology (16). The pathologic reports described T-stage (TNM-staging classification), the shortest distance (in millimeter) between residual tumor and the radial inked resection plane and the shortest distance between tumor and resected organ(s)/ other pelvic structures when present. All pathologic specimen were reviewed by a pathologist specialized in gastroenterologic pathology in order to determine the anatomical orientation of the tumor and its distance to the anatomic plane of the mesorectal fascia. The shortest distance between the tumor and the radial resection plane corresponded with the distance to the mesorectal fascia only for resections which took place along the mesorectal fascia. Therefore, the tumor distance to the mesorectal fascia needed to be reconstructed for resections outside the mesorectal compartment by using clear anatomical landmarks (pelvic organs and other pelvic structures) and carefull review of all clinical, PET/CT and MRI information in combination with the pathologic specimen. All pathologic specimen were also reviewed for the determination of the tumor regression grade (TRG) according to the classification of Mandard (17). TRG is a semiquantitative measure of the proportion of residual tumor load to scarring and therefore pathologic tumor regression expressed on a 5 point scale: TRG $1=$ no residual tumor; TRG $2=$ rare residual cancer cells; TRG $3=$ fibrosis outgrowing residual cancer; TRG $4=$ residual cancer outgrowing fibrosis; TRG $5=$ abcense of regressive changes. 


\section{Reference standard of tumor regression from the mesorectal fascia}

All patients had initial tumor invasion of the mesorectal fascia as determined by pre-CRT MRI in a multi-disciplinary team setting which represents the most reliable tool for the determination of the tumor relationship to the mesorectal fascia prior to neo-adjuvant therapy (18-20). Therefore, a distance of $>2 \mathrm{~mm}$ between residual tumor tissue and the plane of the mesorectal fascia at histologic examination of the surgical specimen was considered as anatomical tumor regression from the mesorectal fascia after chemoradiation treatment (21).

\section{Statistical analysis}

The software package SPSS ${ }^{\circledR} 11.0$ (Chicago, USA) was used All quantitative data are expressed as mean values ( \pm standard deviation) and displayed with $95 \%$ confidence intervals. The Mann Withney $U$ test was used for the assessment of statistical difference between patients with and without tumor regression from the mesorectal fascia for measured SUVmax values, measured distances by using auto-contouring and assessed TRG in the specimen. The same test was used for the determination of statistical difference between patients with TRG 1-2 vs TRG 3-5 for SUVmax values. A two tailed statistical difference of $p<0.05$ was considered as statistical significant. SUVmax value changes after chemoradiation therapy were tested with the Wilcoxon Rank test.

\section{Results}

\section{Histology}

Histologic evaluation of the specimens revealed a tumor free mesorectal fascia and therefore anatomical tumor regression from the mesorectal fascia in $14 / 20(70 \%)$ patients and no tumor regression in $6 / 20(30 \%)$ patients. The mean tumor distance to the mesorectal fascia was $10 \pm 5 \mathrm{~mm}$ (range $4-18 \mathrm{~mm}$ ) in the group of patients with tumor regression from the mesorectal fascia. Two of these 14 patients had a complete pathologic remission (10\% pCR), 7 of 14 showed T1-2 tumors and 5 of 14 patients had T3 tumors. Comparison of patients with and without tumor regression from the mesorectal fascia showed no statistical difference for the tumor regression grade (TRG 2, range 1-4 vs TRG 3, range 2-4; $p>0.1$ ).

\section{Tumor regression grade (TRG) and SUV measurements}

Comparison of the groups of patients with TRG 1-2 (=pathological tumor response) vs TRG 3-5 (=no pathological tumor response) revealed a statistical difference for the percentage of SUVmax reduction (TRG 1-2: 83.0 $\pm 12.0 \%$ SUVmax reduction vs TRG 3-5: $59.4 \pm 24.5 \%$ SUVmax reduction; 
$p=0.025$; figure 2). In contrast, no statistical difference could be identified for SUVmax prior to chemoradiation (TRG 1-2: SUVmax=14.5 \pm 2.5 vS TRG 3-5: SUVmax $=13.7 \pm 5.4 ; p=0.54)$. There was a nearly statistical significant SUVmax difference after chemoradiation (TRG 1-2: SUVmax=3.0 \pm 2.2 VS TRG 3-5: SUVmax $=5.0 \pm 2.0 ; p=0.059$ ).

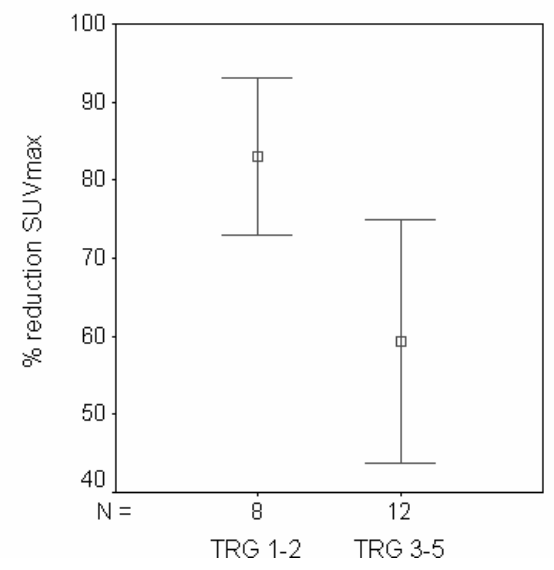

Fig. 2. Calculated percentage (\%) SUVmax reduction with $95 \%$ confidence interval for patients with TRG 1-2 (=pathological tumor responders; $n=8$ ) vs TRG 3-5 (=non responders; $n=12$ ).

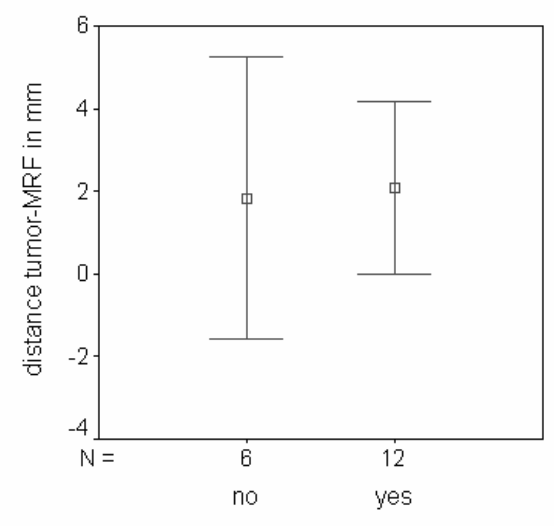

tumor regression from MRF

Fig. 3. Measured shortest distance (in $\mathrm{mm}$ ) with $95 \%$ confidence interval between the outlined tumor volume and the mesorectal fascia after CRT for patients with $(n=12)$ and without $(n=6)$ tumor regression from the mesorectal fascia as determined by pathology.

Note: Two of 20 patients had a complete remission of the tumor according to the auto-contouring software. Therefore, no distance to the MRF could be determined. These two patients were excluded from the statistical analysis. Histologic evaluation showed that one of these patients had a complete pathological remission and the other patient had microscopic tumor disease confined to the rectal wall. 


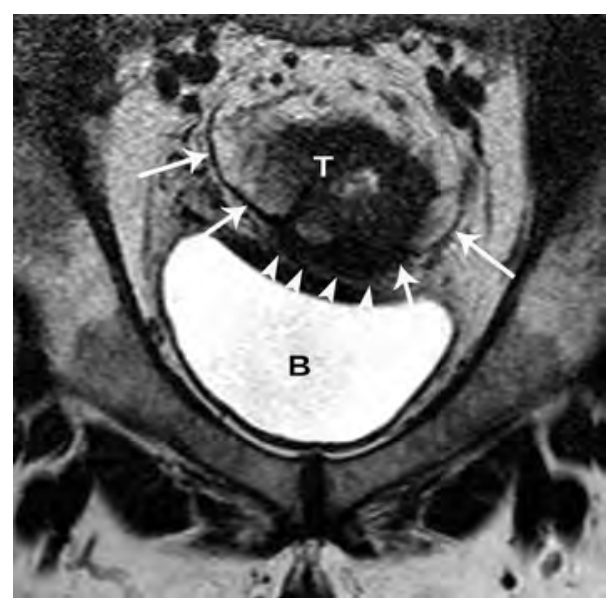

$4 a$

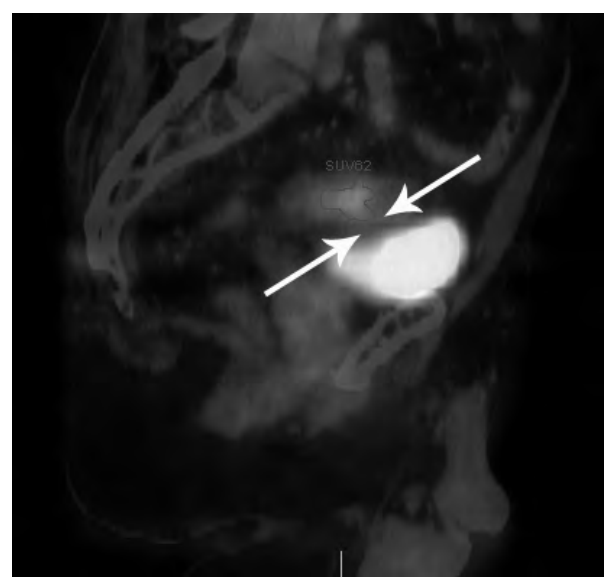

4c

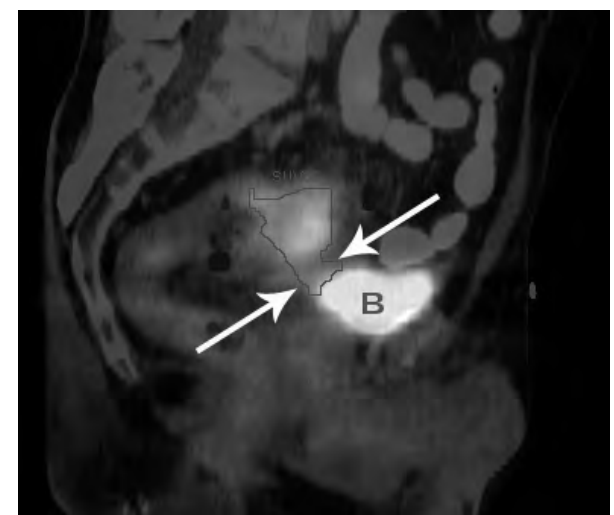

4b

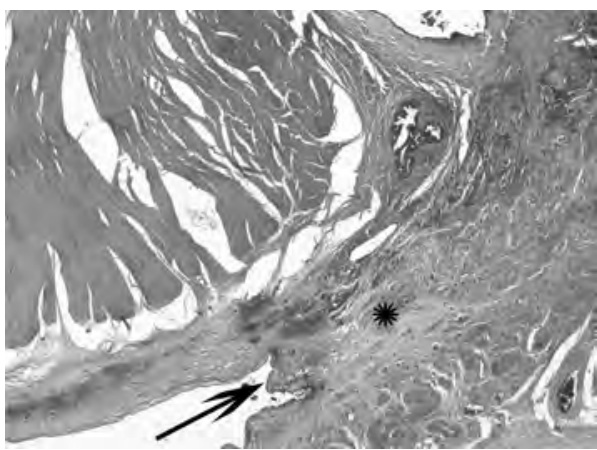

4d

Fig. 4a-d. Example of overstimation of anatomical tumor response by auto-contouring software in areas of microscopic tumor disease. (a) Axial prechemoradiation T2-weighted MR image showing tumor $(\mathrm{T})$ penetration through the anterior mesorectal fascia (arrows) into the bladder roof (arrowheads) ( $B=$ bladder). (b) Sagittal prechemoradiation fused PET/CT image. There is a close relationship (arrows) between the tumor outlined by the auto-contouration software and the bladder roof $(B=$ bladder). (c) Sagittal postchemoradiation fused PET/CT image shows the development of a wide distance inbetween the outlined tumor contour and the bladder as well as anterior mesorectal fascia (arrows) suggesting anatomical tumor regression from the bladder and anterior mesorectal fascia. (d) Corresponding histology shows microscopic tumor disease (asterisk) near the resection margin (arrow). This was considered as a proof of tumor invasion of the mesorectal fascia and perivesical fat because the surgeon performed a wide extramesorectal excision including the bladder roof. 

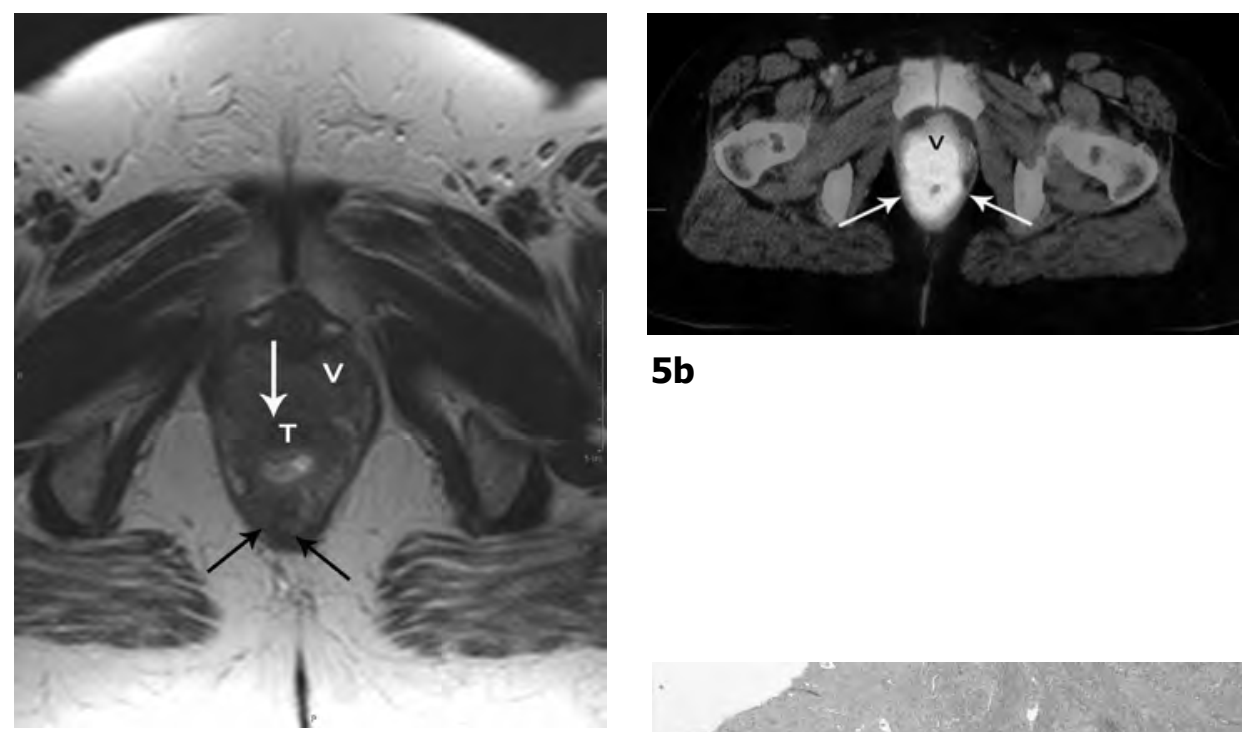

5b

\section{$5 \mathbf{a}$}

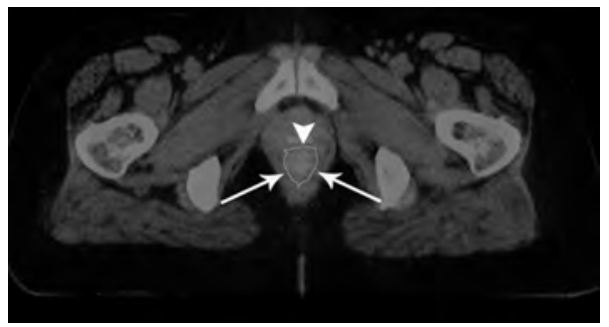

5c

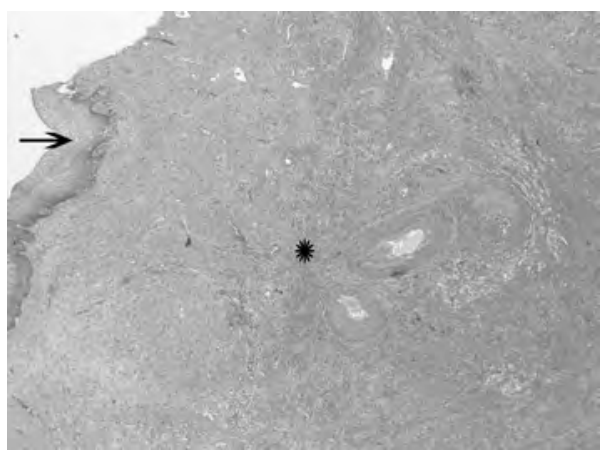

5d

Fig. 5a-d. Example of underestimation of tumor response by auto-contouring software in areas of inflammatory tissue reaction induced by chemoradiation.therapy (a) Axial prechemoradiation T2-weighted MR image showing clear tumor penetration (arrow) through the anterior mesorectal fascia into the vagina ( $T=$ tumor; $V=$ vagina) and there is close tumor contact with the pelvic floor muscles (black arrows). (b) Axial prechemoradiation auto-contoured PET/CT image. The anterior mesorectal fascia and vagina (V) is seen within the outlined tumor volume and there is close contact with the pelvic floor muscles (arrows). (c) Axial postchemoradiation PET/CT image showing tumor volume shrinkage but there is still contact between the auto-contoured tumor volume and the vagina (arrowhead) as well as the pelvic floor muscles (arrows). (d) corresponding histology shows no tumor but only an inflammatory reaction (asterisk) at the level of the vagina (arrow $=$ mucosal surface of vagina). 


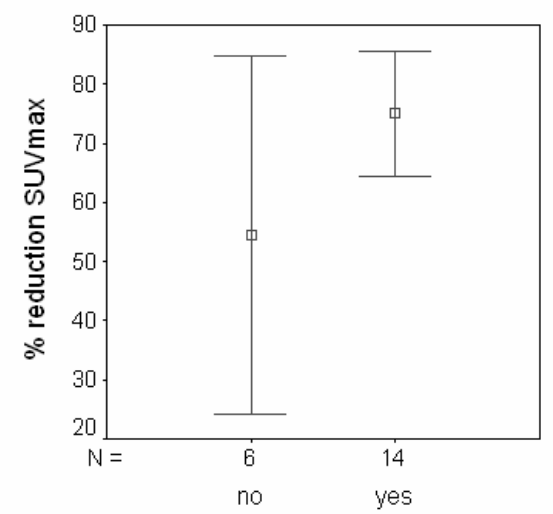

$6 a$

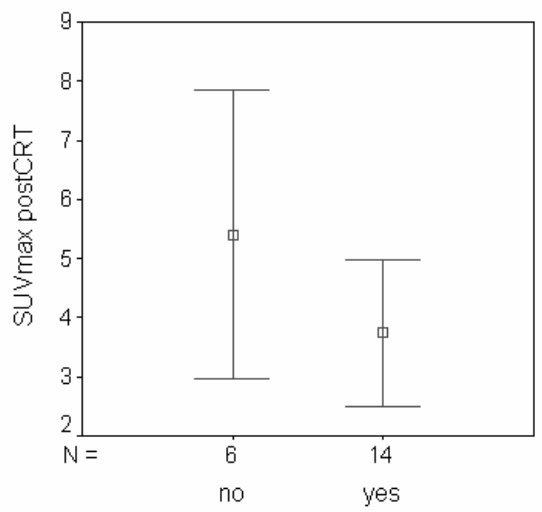

6b tumor regression from MRF

Fig. 6a-b. Calculated percentage SUVmax reduction (a) and measured SUVmax after chemoradiation (b) with $95 \%$ confidence interval for patients with $(n=6)$ and without $(n=14)$ tumor regression from the mesorectal fascia as determined by pathology.

\section{Tumor regression from the mesorectal fascia and auto-contouring}

The measured shortest distances between the outlined tumor volumes and the mesorectal fascia for patients with and without tumor regression from the mesorectal fascia are displayed in figure 3. The mean distance was $2.1 \pm 3 \mathrm{~mm}$ (range $0-8 \mathrm{~mm}$ ) for patients with tumor regression from the mesorectal fascia and $1.8 \pm 3 \mathrm{~mm}$ (range $0-8 \mathrm{~mm}$ ) for patients without tumor regression from the mesorectal fascia with no statistical difference $(p>0.1)$.

Figures $4 a-d$ and $5 a-d$ illustrate pitfalls which were observerd for the determination of tumor regression from the mesorectal fascia by use of autocontouring software.

\section{Tumor regression from the mesorectal fascia and SUV measurements}

The measured SUVmax values for patients with and without tumor regression from the mesorectal fascia are displayed in figure $6 a-b$. The mean SUVmax value for patients with tumor regression from the mesorectal fascia was calculated as $14.2 \pm 4.4$, dropping by $75.0 \pm 18.4 \%$ to $3.7 \pm 2.1$ after CRT. For patients without tumor regression, the mean SUVmax was 13.4 \pm 4.6 , dropping by $54.5 \pm 28.9 \%$ to $5.4 \pm 2.3$ after chemoradiation. There was a statistical significant SUVmax reduction in both groups of patients $(p=0.001)$ but no statistical difference could be found between these groups of patients for SUVmax pre- and post-CRT as well as percentage of SUVmax reduction $(p=0.5,0.08$ and 0.2 respectively). 


\section{Discussion}

The present study evaluated the value of repeated PET/CT after chemoradiation in predicting tumor regression from the mesorectal fascia in initially locally advanced rectal cancer patients Our data demonstrated a statistical significant correlation between the percentage SUVmax reduction and the pathologic tumor response, as expressed by the TRG score and a trend between the postCRT SUVmax and the TRG. However, none of the tested $\mathrm{PET} / \mathrm{CT}$ parameters, like measured shortest tumor distance to the mesorectal fascia by use of auto-contouring software, the percentage SUVmax reduction or the pre- and post therapeutic SUVmax were able to predict the pathologically proven tumor regression from the mesorectal fascia.

Our data confirm previous publications that pathological tumor response can be predicted by PET (22-25). Caprici et al. reported in a study of 45 rectal cancer patients a statistical difference in the percentage of SUVmax reduction between responders classified as TRG 1-2 (76\% SUVmax reduction) and non responders defined as TRG 3-5 (47\% SUVmax) (22). The same author showed in a different study of 81 rectal cancer patients a statistical correlation between visual assessed PET response and TRG (23). In a study of 33 patients, Cascani and colleagues showed a statistical difference in early decrease of SUVmax between responders (=TRG 1-2) and non responders classified as TRG $3-4$ (62\% SUVmax reduction vs $22 \%$ ) (24). Melton et al. found in a study of 21 patients that complete pathological response or microscopic tumor disease was associated with greater changes in measured SUV (25). These findings support that tumor cell killing (pathological tumor response) by effective chemoradiation can be non-invasively monitored by changes in functional PET parameters. This may have important clinical implications as these findings suggest that adjustments of neo-adjuvant therapy regimes can be contemplated in non responders identified by PET.

Our method of auto-contourating has been established in phantom studies with radio-active spheres and successfully tested in previously untreated cancers of various origins (14-15, 26-27). Current available information on this subject suggests a potential role of this technique for radiotherapy planning by improving inter-observer agreement and a better and more uniform definition of the target volume. In a recent prospective study of head and neck cancer patients published by Daisne and colleagues, the tumor volumes as determined by automated contouring of PET matched far the closest with the actual tumor volume of the surgical specimen, compared to CT and MRI (26). In a study of non small cell lung cancer, Van Baardwijk et al. found a strong correlation between the tumor diamer measured by auto-contouring (correlation coefficient $=0.90$ ) and macroscopic tumor diameter at histology (15). The only PET auto-contouring study in rectal cancer patients performed by Ciernik et al. found a moderate correlation (correlation coefficient=0.77) between tumor 
diameters determined by auto-contouring and histology although tumor shrinkage after PET imaging due to radiotherapy interfered with the reference standard histology (27).

In addition to already existing knowledge, our experience with autocontouring of PET/CT information showed that it is a poor indicator of anatomical tumor changes (as in this study tumor regression from the mesorectal fascia) after chemoradiation therapy.The main reasons are the obvious complex tumor shapes and constitution of microscopic tumor disease scattered in a background of regressive tissue changes which is a quite different situation compared to previously untreated tumors. Microscopic tumor disease at the tumor's boarder appears to be an extreme challenge for automated determination of tumor contours. Insufficient FDG uptake of these small tumor nests beyond any detection level of todays PET techniques obviously results in an underestimation of the actual tumor volume and therefore an overestimation of anatomical tumor response as we observed. This finding is supported by a recent PET study of Caprici et al. showing small residual tumor load in as high as $53 \%(23 / 43)$ of rectal cancer patients without identifiable FDG-tracer uptake (23). In fact, these findings indicate that adjusting the surgical radicality in previous tumor areas which have become PET negative must be considered as unsafe. It will expose patients to incomplete surgical resections of tumor disease which is considered as unacceptable due to its high associated risk of local tumor recurrence (28). Also, one has to be aware of an obvious risk of too much narrowing the irradiated target volume when adjustments of the target volume are made during the course of intensity modulated radiotherapy based on PET/CT information. In addition to potential underestimation of the tumor volume, we also observed quite the opposite. Increased uptake of FDG tracer in areas of inflammatory reaction resulted in overestimation of the actual tumor volume and therefore underestimation of the anatomical tumor response. It is clear that this false positive uptake of FDG tracer ultimately leads to surgical overtreatment which is currently also one of the major concerns in conventional CT and MRI imaging (10-12). Increased glucose uptake by inflammatory reaction has been generally recognized as a confounding factor of PET response evaluation across different tumor types starting from 1-2 weeks up to 6 months after the end of radiation therapy although some of the PET studies in rectal cancer patients have debated major adverse effects $(23,29-33)$. This controversy stresses the importance to further evaluate the impact of neo-adjuvant treatment on the PET response evaluation, even more with the recent introduction of new drugs. A proposed waiting time of 6 months to overcome potential confounding effects of neo-adjuvant treatment is certainly not practical in todays treatment in which neo-adjuvant treatment is usually followed by surgery in 6-8 weeks (31). 
In contrast to other literature our study showed that SUVmax changes measured after chemoradiation were a poor indicator of anatomical tumor changes $(13,22,32,34)$. Changes in measured functional PET parameters are related to tumor cell killing which on itself is not necessarily accompanied by tumor size reduction $(29,35)$. We found a comparable degree of tumor cell killing expressed by similar TRGs (not statistical different) in both patients with and without tumor regression from the mesorectal fascia which actually determined the measured metabolic changes by PET. This could explain why we found no statistical difference in measured SUVmax between both groups of patients, the latter supporting the finding that PET is a poor indicator of anatomical changes in rectal tumor treated by chemoradiation.

When analyzing the data of the present study certain limitations have to be taken into account. First, auto-contouring of the tumor volumes occurred on a sub-grid scale i.e. below the PET pixel resolution of approximately $4 \mathrm{~mm}$. This suboptimal spatial resolution would have been certainly a limitation for the determination of the anatomical relationship of tumors with a close distance to to the mesorectal fascia due to partial volume effects. However, the gross topographic discrepancies between the tumor load located at far (>5-10 mm) distances from the mesorectal fascia as determined by histologic examination of the specimens and the displayed close relation by the auto-contouring software supported that this was not an important issue. Our phantom study of concentric spheres as a model of the mesorectal anatomy confirmed the high geometrical acuracy of the auto-contouring software for the determination of the relationship between tumor and the mesorectal fascia. A second limitation of this study is related to the reference standard for patients who received resection outside the confines of the mesorectal compartment and therefore the tumor relation to the mesorectal fascia needed to be reconstructed. In order to keep potential inaccuracies as low as possible, all available clinical, histopathologic, PET/CT and MRI information were carefully reviewed. Further, we are aware that in some of our patients in reality, the tumor could have been close to rather than invading the MRF prior to the application of chemoradiation therapy as determined by MRI. Because of the neo-adjuvant chemoradiation treatment, the traditional reference standard histology is no longer valid for the determination of the initial tumor stage due to the tumor downstaging and sizing effect. Therefore, we used MRI which is the present standard for the determination of the tumor relationship to the mesorectal fascia (18-20). Finally, our study was based on a rather small number of patients and therefore our results need to be confirmed in larger prospective studies. Especially the exact relationship between neo-adjuvant therapy type and confounding effects of inflammatory reactions needs to be clarified. However, evidence already suggests a clear limitation of PET to detect microscopic tumor disease which is an obvious major limitation for the determination of a surgical road map in patients treated with chemoradiation $(13,23,32)$. 


\section{Conclusion}

Our data suggest that PET/CT is a poor indicator for anatomical changes in rectal tumors after neo-adjuvant chemotherapy and therefore not accurate for the prediction of tumor regression from the mesorectal fascia. However PET/CT is a good indicator for pathological tumor response enabling early differentiation between responders and non responders to neo-adjuvant chemoradiation

\section{References}

1. Sauer R. Becker $H$, Hohenberger $W$, et al. Preoperative versus postoperative chemoradiotherapy for rectal cancer. N Engl J Med 2004; 351:1731-1740.

2. Palmer G, Martling A, Blomqvist L, et al. Outcome after the introduction of a multimodality treatment program for locally advanced rectal cancer. Eur J Surg Oncol 2005; 31:727. 734.

3. Govindarajan A, Coburn N, Kiss A, et al. Population based assessment of the surgical management of locally advanced rectal cancer. J Natl Cancer Inst 2006; 98:1474-1481.

4. Lehnert T, Methner M, Pollok A, et al. Multivisceral resection for locally advanced primary colon and rectal cancer: an analysis of prognostic factors in 201 patients. Ann Surg 2002; 235:217-225.

5. Rodel C, Sauer R. Integration of novel agents into combined-modality treatment for rectal cancer patients. Strahlenther Onkol 2007; 183:227-235.

6. Bonnen M, Crane C, Vauthey J, et al. Long-term results using local excision after preoperative chemoradiation among selected T3 cancer patients. Int J Radiat Oncol Biol Phys 2004; 60:1098-1105.

7. Habr-Gama A, Perez R, Nadalin W, et al. Operative versus nonoperative treatment for stage 0 distal rectal cancer following chemoradiation therapy: long-term results. Ann Surg 2004; 240:711-717.

8. Chau I, Brown G, Cunningham D, et al. Neoadjuvant capecitabine and oxaliplatin followed by synchronous chemoradiation and total mesorectal excision in magnetic resonance imaging-defined poor- risk rectal cancer. J Clin Oncol 2006; 24:668-674.

9. MacFarlane J, Ryall R, Heald R. Mesorectal excision for rectal cancer. Lancet 1993; 341:457-460.

10. Kuo $L$, Chern M, Tsou M, et al. Interpretation of magnetic resonance imaging for locally advanced rectal carcinoma after preoperative chemoradiation therapy. Dis Colon Rectum 2005; 48:23-28.

11. Hoffmann K, Rau B, Wust $P$, et al. Restaging of locally advanced carcinoma of the rectum with MR imaging after preoperative radio-chemotherapy plus regional hyperthermia. Coloproctology 2002; 24:253-261.

12. Chen $C$, Lee $R$, Lin J, et al. How accurate is magnetic resonance imaging in restaging rectal cancer in patients receiving preoperative combined chemoradiotherapy? Dis Colon Rectum 2004; 48:722-728.

13. Denecke T, Rau B, Hoffmann K, et al. Comparison of CT, MRI and FDG-PET in response prediction of patients with locally advanced rectal cancer after multimodal preoperative therapy: Is there a benefit in using functional imaging? Eur Radiol 2005; 15:1658-1666.

14. Daisne J, Sibomana M, Bol A, et al. Tri-dimensional automatic segmentation of PET volumes based on measured source-to-background ratios: influence of reconstruction algorithms. Radiother Oncol 2003; 69:247-250. 
15. van Baardwijk A, Bosmans G, Boersma L, et al. PET-CT-based auto-contouration in nonsmall-cell lung cancer correlates with pathology and reduces interobserver variability in the delineation of the primary tumor and involved nodal volumes. Int J Radiat Oncol Biol Phys 2007; 68:771-778.

16. Quirke $\mathrm{P}$, Dixon $\mathrm{M}$. The prediction of local recurrence in rectal adenocarcinoma by histopathological examination. Int J Colorectal Dis 1988; 3:127-131.

17. Mandard A, Dalibard F, Mandard J, et al. Pathologic assessment of tumor regression after preoperative chemoradiotherapy of esophageal carcinoma. Cancer 1994; 73:2680-2686.

18. Beets-Tan R, Beets $G$, Vliegen $R$, et al. Accuracy of magnetic resonance imaging in prediction of tumour-free resection margin in rectal cancer surgery. The Lancet 2001; 357:497-504.

19. MERCURY study group. Diagnostic accuracy of preoperative magnetic resonance imaging in predicting curative resection of rectal cancer: prospective observational study. BMJ 2006; 333:779-790.

20. Lahaye $M$, Engelen $S$, Nelemans $P$, et al. Imaging for predicting the risk factors-the circumferential resection margin and nodal disease--of local recurrence in rectal cancer: a meta-analysis. Semin Ultrasound CT MR 2005; 26:259-268.

21. Nagtegaal I, Marijnen C, Kranenbarg E, et al. Circumferential margin involvement is still an important predictor of local recurrence in rectal carcinoma: not one millimeter but two millimeters is the limit. Am J Surg Pathol 2002; 26:350-357.

22. Capirci $C$, Rampin L, Erba $P$, et al. Sequential FDG-PET/CT reliably predicts response of locally advanced rectal cancer to neo-adjuvant chemo-radiation therapy. Eur J Nucl Med Mol Imaging 2007; 34:1583-1593.

23. Capirci $C$, Rubello D, Chierichetti $F$, et al. Restaging after neoadjuvant chemoradiotherapy for rectal adenocarcinoma: role of F18-FDG PET. Biomed Pharmacother 2004; 58:451457.

24. Cascani L, Avallone A, Delrio P, et al. 18F-FDG PET is an early predictor of pathologic tumor response to preoperative radiochemotherapy in locally advanced rectal cancer. J Nucl Med 2006; 47:1241-1248.

25. Melton $G$, Lavely $W$, Jacene $H$, et al. Efficacy of preoperative combined 18Fluorodeoxyglucose Positron Emission Tomography and Computed Tomography for assessing primary rectal cancer response to neoadjuvant therapy. J Gastrointest Surg 2007; 11:961-969.

26. Daisne J, Duprez T, Weynand B, et al. Tumor volume in pharyngolaryngeal squamous cell carcinoma : comparison at CT, MR imaging, and FDG PET and validation with surgical specimen. Radiology 2004; 233:93-100.

27. Ciernik I, Huser M, Burger $C$, et al. Automated functional image-guided radiation treatment planning for rectal cancer. Int. J. Radiat Oncol Biol Phys 2005; 62:893-900.

28. Luna-Perez P, Bustos-Cholico E, Alvarado I, et al. Prognostic significance of the circumferential margin involvement in rectal adenocarcinoma treated with preoperative chemoradiotherapy and low anterior resection. J Surg Oncol 2005; 90:20-25.

29. Young $\mathrm{H}$, Baum $\mathrm{R}$, Cremerius $\mathrm{U}$, et al. Measurement of clinical and subclinical tumour response using [18F]-fluorodeoxyglucose and positron emission tomography: review and 1999 EORTC recommendations. Eur J Cancer 1999; 35:1773-1782.

30. Van Ginkel R, Hoekstra H, Pruim J, et al. FDG-PET to evaluate response to hyperthermic isolated limb perfusion for locally advanced sift tissue sarcoma. J Nucl Med 1996; 37:984990.

31. Haberkorn U, Strauss L, Dimitrakopoulou A, et al. PET studies of fluorodeoxyglucose metabolism in patients with recurrent colorectal tumours receiving radiotherapy. J Nucl Med 1991; 32:1485-1490. 
32. Amthauer $\mathrm{H}$, Denecke $\mathrm{T}$, Rau B, et al. Response prediction by FDG-PET after neoadjuvant radiochemotherapy and combined regional hyperthermia of rectal cancer: correlation with endorectal ultrasound and histopathology. Eur J Nucl Med Mol Imaging 2004; 31:811-819.

33. Guillem J, Puig-La Calle J, Akhurst T, et al. Prospective assessment of primary rectal cancer response to preoperative radiation and chemotherapy using 18-fluordeoxyglucose positron emission tomography. Dis Colon rectum 2000; 43:18-24.

34. Calvo F, Domper M, Matute R, et al. F-FDG positron emission tomography and restaging in rectal cancer terated with preoperative chemoradiation. Int. J. Radiat Oncol Biol Phys 2004; 58:528-535.

35. Schiepers C, Haustermans K, Geboes K, et al. The effect of preoperative radiation therapy on glucose utilization and cell kinetics in patients with primary rectal carcinoma. Cancer 1999; 85:803-811. 


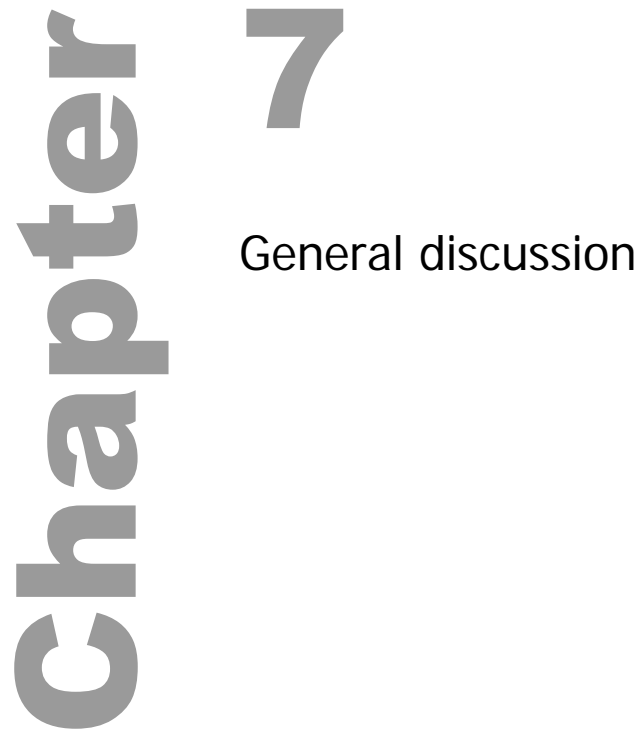




\section{General discussion}

This thesis reports on the local staging and restaging (after neo-adjuvant therapy) capabilities of different cross sectional imaging techniques and their potential role in the diagnostic work-up of rectal cancer patients. The impetus for these studies have been the recent developments in the field of imaging techniques, a call for optimization of already established diagnostic techniques and upcoming new treatment strategies for rectal cancer with an impact on imaging and vica versa.

\section{Optimization of the Magnetic Resonance Imaging (MRI) protocol for rectal cancer staging}

MR imaging has been established as first choice diagnostic modality for rectal cancer staging (1-5). Different MR imaging protocols have been succesfully used for this purpose but studies on the comparison have been scarce (6-9).

Our study described in chapter $\mathbf{3}$ was the first in addressing the value of gadolinium enhanced T1-weighted images as an adjunct to T2-weighted Fast Spin Echo (FSE) MR imaging sequences for predicting the clinical important tumor relationship to the mesorectal fascia.

Our data demonstrated a high performance of T2-weighted FSE sequences (Area Under the ROC curves of 0.90-0.96). The addition of gadolinium enhanced images did not improve the staging accuracy of MR imaging.

Pitfalls experienced on T2-weighted sequences such as the difficult interpretation of tissue strands close to the mesorectal fascia remain on whatever MR imaging sequence is applied. The identification of microscopic tumor load within some of these fibrotic strands is beyond the detection level of MRI and both strands with and without tumor load enhance after the the application of gadolinium.

The clinical significance of our findings is obvious. Gadolinium enhanced sequences can be omitted. It saves valuable MR imaging time, reduces costs and prevents nefrogenic systemic fibrosis, a disease which has been recently linked to the use of gadolinium contrast media in patients with renal insufficiency (10).

A standard MR imaging protocol consisting of T2-weighted FSE sequences alone performs well and this is presently considered as standard in rectal cancer imaging (1,11-12). However, this standard protocol may change, influenced by ongoing technical developments. Recently available T2-weighted volume scans may be of benefit for the evaluation of difficult anatomical regions such as the distal rectum. These sequences can generate images which can be reconstructed in every chosen plane at any time. Furthermore, high field 
strengths machines and new coil designs may improve anatomical detail which may solve the staging problems of superficial tumors experienced at 1.5 Tesla systems with external coils (13-14). Even a comeback of contrast agents must be considered as realistic but their indications are different. Upto now, the attention was mainly focussed on the prediction of tumor invasion of the circumferential resection margin and not on lymph node metastases, another adverse risk factor for local recurrence. The main reason was that accurate prediction of the lymph node status by conventional cross sectional imaging has been difficult (4). There are now however new specific contrast agents like USPIO (ultrasmall superparamagnetic iron oxide particles) that show promising results for the prediction of lymph node metastatic disease (15-16). Information provided by these new agents may become important determinants in treatment strategy decisions. Also, recent achievements of dynamic contrast enhanced MRI (DCE-MRI) may lead to a reconsideration of current standard non-contrast MR protocols. Preliminary data suggest that this functional MR technique gives insight in microvascular changes in the tumor tissue such as angiogenesis and tumor hypoxia (17-19). This information may become important for monitoring and individualization of anti-tumor strategies. The exact role of this technique and new contrast media such as blood pool agents needs to be determined.

\section{The role of Multi-Detector row Computed Tomography (MDCT) as an alternative to MRI for local rectal cancer staging}

The quick acquisition of a detailed whole body volume data set is a clear advantage of MDCT for the determination of an integral treatment plan. Although the staging capabilies of MDCT for metastatic colorectal disease such as liver metastasis have been well studied, little information can be found on the important prediction of the tumor relationship to the mesorectal fascia (20). It is obvious that when MDCT is a good alternative to MRI, its performance for the prediction of mesorectal fascia invasion should be comparable to the present standard MRI.

In chapter 4, we evaluated the value of MDCT for the prediction of tumor invasion of the mesorectal fascia at patient level, as well as at different anatomical locations of the mesorectal compartment.

Our results showed a reasonable high accuracy of MDCT for the prediction of tumor invasion of the mesorectal fascia for proximally located rectal tumors (Area Under the ROC Curves of $0.84-0.88$ ), but the staging accuracy appeared to be poor for distal tumors $(A \cup C=0.31-0.50)$.

One of the main reasons of the staging problems experienced with distal tumors is the suboptimal soft tissue contrast resolution of CT, related to the principles of this modality. This limitation is responsible for the provision of 
an insufficient anatomical detail which is critical in difficult regions with a close anatomy such as the distal rectum.

Our results have since been confirmed by studies of conventional spiral CT and MDCT (21-22). Therefore, based on the present available information, CT can not be considered as a good alternative to MRI for the prediction of tumor invasion of the mesorectal fascia, especially not for staging distal tumors. Maybe one of the exceptions is when MRI for what reason is contra-indicated or unavailable and the tumor is expected to be located proximally in the rectum.

However, we must add a critical note when considering that MDCT techniques have improved during the time span of our study. Presently, MDCT techniques use data sets acquired with submilimeter collimation and optimized contrast phases obtained by scanners with 64 detector rows. These data sets are viewed in reconstructed multiple planes (MPR). These state of the art techniques may improve the staging results of $\mathrm{CT}$, and this has to be evaluated in the future (23). The question nevertheless remains if these optimized MDCT techniques will ever approximate the almost unsurpassable high soft contrast resolution of MRI, as previously discussed crucial for local staging of distal tumors.

Maybe a point of greater concern is how to deal with the prediction of the clinical important lymph node status on $\mathrm{CT}$. Despite ongoing technical improvements and the application of optimized protocols a reliable prediction will be unlikely. The applied iodine contrast medium is non specificic for lymph nodes and therefore the only clue for lymph node metastasis remains the size of the lymph node, known to be unreliable (24). Also in this respect, MRI is far ahead due to the introduction of lymph node specific MR contrast media (USPIO), previously discussed. This contrast agent is bound to solve the problem of lymph node staging and therefore further establishing the role of MRI as first choice staging tool in rectal cancer.

\section{The role of MRI for assessing tumor response after chemoradiation treatment}

The introduction of effective chemoradiation therapy and their high response rates have provoked a wide discussion considering less extensive surgical resections for good responders (25-27). Crucial in this discussion is a reliable tool which can provide a road map for residual tumor disease, used for planning more conservative surgical resections in responders.

In chapter 5, we studied the value of MRI to identify patients with tumor invasion of the mesorectal fascia after neo-adjuvant chemoradiation treatment: a relevant question which has never been addressed before.

Our results showed that postchemoradiation MRI has a high negative predictive value (NPV) of $100 \%$ but a moderate positive predictive value $( \pm 50 \%)$ for predicting tumor invasion of the mesorectal fascia. This means that 
MRI is able to identify patients with tumors that have regressed from the mesorectal fascia with a high NPV, and these patients may be safely treated with total mesorectal excision surgery. However, it fails to identify a substantial number of responders, who are subsequently exposed to wide area resections despite good tumor response, as shown by the moderate positive predictive value.

The main reason of this limitation of $M R I$ is the problematic interpretation of diffuse areas of postchemoradiation "fibrotic tissue", an MR imaging feature seen in more than $50 \%$ of our patients. The problem is that residual tumor within these fibrotic areas is often confined to small tumor nests, beyond the detection level of MRI. So, it is therefore impossible to differentiate these from completely sterilized areas of fibrosis on MRI.

The use of dynamic contrast enhanced sequences has been reported to be useful in other applications such as differentiating tumor (recurrences) from benign (postoperative) tissue by measuring time intensity curves or perfusion indices (28-29). However, it remains questionable whether these techniques would offer any help for the problematic interpretation of diffuse "fibrotic" areas: the detection of small tumor nests within poorly vascularized fibrotic tissue. Other modalities, even Positron Emission Tomography (PET) suffer from the same limitation as illustrated by recent PET publication that reported in the majority of responding tumors with small residual tumor disease no FDG tracer uptake (30).

Despite these limitations, we find that postchemoradiation MRI has earned a place in the standard work-up of locally advanced tumors. Patients with a good response can be selected based on morphological MR patterns, defined in our study. Based on these tumor changes seen on MRI, surgeons get informed of a high, intermediate or low risk for tumor invasion of the mesorectal fascia after chemoradiation. It is clear that this information is of benefit for planning the extent of the surgical resection.

\section{The role of Positron Emission Tomography-CT (PET-CT) for assessing tumor response after chemoradiation treatment}

PET has been claimed to be superior to conventional techniques like CT and MRI for the prediction of tumor response in rectal cancer patients treated with neo-adjuvant chemoradiation (31). This potential has been suggested to be of use to identify good responders for more conservative surgical therapy (30-35). Up to now, PET studies have focussed on the prediction of histopathologic tumor response and not on the prediction of anatomical tumor regression such as tumor regression from the mesorectal fascia (32-35). It is obvious that this question is relevant when more conservative surgery is contemplated. 
In chapter 6, we studied the value of PET-CT for the identification of patients with tumor regression from the mesorectal fascia after neo-adjuvant chemoradiation treatment. Patients underwent PET-CT before and after chemoradiation therapy. The maximal Standard Uptake Values (SUVmax) were determined before and after chemoradiation and the shortest distance between the automatically outlined tumor volume and the mesorectal fascia was measured after the application of chemoradiation therapy.

Our results showed that PET-CT was not able to differentiate between patients with and without tumor regression from the mesorectal fascia, neither by using automated tumor contouring software nor by any of the tested SUV parameters. These findings suggest that the value of PET-CT to plan more conservative surgical resections is limited.

Measured SUV changes on PET-CT examinations are poor indicators of anatomical tumor changes such as tumor regression from the mesorectal fascia. In fact, SUV changes are related to tumor cell killing which may or may not be accompanied with a certain reduction in tumor size or stage but these anatomical changes are basically not measured by PET (36-37). Therefore, they can not be predicted on PET.

Certain limitations with an impact on the evaluation of anatomical tumor response have to be taken into account when considering the suboptimal spatial resolution of available PET scanners. Although improvements of the spatial resolution are expected from the next generation PET scanners and the integration of modalities such as PET and MRI, it will not solve the main limitations experienced with (automated) tumor delineation in patients treated with chemoradiation. The main issues are related to the aspecific nature of the FDG tracer showing false positive increased uptake in inflammatory areas and the inability of PET to detect microscopic tumor disease $(33,38-39)$. Both limitations are often encountered in responding tumors treated with chemoradiation in which microscopic tumor disease is scattered in a background of fibrotic and inflammatory tissue changes. This difficult presentation of responding tumors obviously results in an under and overestimation of the residual tumor volume by PET in patients treated with long courses of chemoradiation therapy.

Nevertheless, there is a potential role of PET in the diagnostic work-up of rectal cancer. As shown by our results confirming previous PET studies, SUVmax changes correlate with the tumor regression grade (TRG), representing the degree of tumor cell killing induced by the chemoradiation therapy (32-35). This information may become clinical relevant as adjustments early in the course of neo-adjuvant treatment may be accomplished in non responders identified by PET. However, this capability to identify responders early in the course of neo-adjuvant treatment is not exclusive for PET. Preliminary reports of dynamic contrast enhanced MRI have shown a correlation between parameters derived from this functional MR technique and therapy induced 
tissue changes (17-19). Functional MRI may therefore compete with PET-CT and studies on the comparison of these techniques need to be undertaken.

\section{Conclusions}

1. Gadolinium-enhanced sequences do not improve the diagnostic accuracy of MRI for predicting tumor invasion of the mesorectal fascia.

2 MDCT has a poor accuracy for the identification of tumor invasion of the mesorectal fascia in distal rectal tumors. The accuracy of CT significantly improves for staging proximally located rectal tumors.

3. Postchemoradiation MRI has a moderate accuracy for predicting tumor invasion of the mesorectal fascia (MRF) related to the inherent limitation to differentiate between diffuse "fibrotic" tissue harbouring small tumor foci and completely sterilized areas of fibrosis. Specific other types of morphologic tissue changes on MRI can highly predict a tumor free or invaded MRF.

4. PET-CT is a poor indicator for anatomical changes (downsizing and staging) in tumors treated with chemoradiation and therefore not accurate for predicting tumor regression from the mesorectal fascia. Nevertheless, PET-CT is a good indicator for pathological tumor response enabling early differentiation between responders and non responders to neo-adjuvant chemoradiation.

\section{Recommendations}

An optimal MR imaging protocol for rectal cancer staging consists of T2-weighted Turbo Spin Echo without gadolinium enhanced sequences. This applies when the purpose of the MRI is to judge the relation of the primary tumor to the mesorectal fascia. This may change in the future if lymph node imaging becomes important.

For primary staging of rectal cancer, high resolution MRI is the prefered imaging modality. If MRI is unavailable or contra-indicated, MDCT can be considerd as an alternative to MRI for the selection of tumors located in high rectum with a wide tumor free margin. For all other rectal tumors including distal tumors, effective staging capabilities of MDCT have not been proven yet. For these tumors, the staging accuracy of state of the art MDCT and viewing the dataset in multi-planar reconstructions should be explored. However, despite improvements in CT technique, one has to take into acount the limitation of unreliable assessment of metastatic lymph node disease by CT.

Introduction of effective chemoradiation therapy with high response rates, downsizing and staging even to a complete pathological tumor remission, have opened a debate whether or not to resect previously involved organs and whether or not local excision, or wait and see strategies are justified. In these 
decisions, the role of imaging is important. At present MRI can be helpful in selected cases as a restaging tool for the assessment of downstaging and sizing but the prediction of a complete pathological remission remains difficult. This remains problematic because conventional MRI will never be able to identify microscopic tumor disease within areas of chemoradiation induced fibrosis. We believe that if imaging of small amounts of viable tumor cells and its differentiation from inflammatory tissue is ever to be successful, it will be through the advances of molecular imaging. Experience with selective antibodies conjugated to gadolinium have already shown selective accumulation in tumor cells (40). This suggests that these new generation of specific MR contrast agents are potentially suitable to detect local recurrence at an earlier stage as well as to identify areas of tumor within a background of regressive and reactive tissue reaction after chemoradiation treatment. It is clear that it has high priority to further invest in new methods of molecular imaging and add a new functional imaging dimension to conventional anatomical imaging.

PET is a strong predictor of pathological tumor response (tumor cell killing) but the preoperative differentiation between responders versus non responders during or shortly after the course of chemoradiation has currently no therapeutical impact. The prediction of tumor downsizing and staging (anatomical tumor response) by PET-CT is poor and therefore there is currently no role of PET-CT for the preoperative restaging of rectal cancer patients treated with neo-adjuvant chemoradiation.

Although not a subject of our studies, PET-CT could play a role in radiotherapeutical planning. Studies of patients with lung cancer and patients with head and neck tumors have shown that the gross tumor volume determined by automated contouration of PET-CT information correlates better with histology than with conventional imaging methods and therefore this should be evaluated for rectal cancer (41-42). Also, PET may provide additional information by the introduction of specific tracers such as proliferation and hypoxic tracers and open the way for a so called dose painting and dose escalation in hypoxic areas of the tumor.

\section{References}

1. MERCURY study group. Diagnostic accuracy of preoperative magnetic resonance imaging in predicting curative resection of rectal cancer: prospective observational study. BMJ 2006; 333:779-790.

2. Beets-Tan R, Beets $G$, Vliegen $R$, et al. Accuracy of magnetic resonance imaging in prediction of tumour-free resection margin in rectal cancer surgery. The Lancet 2001; 357:497-504.

3. Engelen S, Beets G, Beets-Tan R. Role of preoperative local and distant staging in rectal cancer. Onkologie 2007; 30:141-145.

4. Lahaye $M$, Engelen $S$, Nelemans $P$, et al. Imaging for predicting the risk factors-the circumferential resection margin and nodal disease-of local recurrence in rectal cancer: a meta-analysis. Semin Ultrasound CT MR 2005; 26:259-268. 
5. Valentini V, Glimelius B, Minsky B, et al. The multidisciplinary rectal cancer treatment: main convergences, controversial aspects and investigational areas which support the need for an European consensus. Radiother Oncol 2005; 76:241-250.

6. Okizuka H, Sugimura K, Yoshizako $T$, et al. Rectal carcinoma: prospective comparison of conventional and gadopentetate dimeglumine enhanced fat-suppressed MR imaging. J Magn Reson Imaging 1996; 6:465-471.

7. Maier A, Kersting-Sommerhoff B, Reeders J, et al. Staging of rectal cancer by doublecontrast MR imaging using the rectally administered superparamagnetic iron oxide contrast agent ferristine and IV gadodiamide injection: results of a multicenter phase II trial. J Magn Reson Imaging 2000; 12:651-660.

8. Vogl T, Pegios W, Mack M, et al. Accuracy of staging rectal tumors with contrastenhanced transrectal MR imaging. Am J Roentgenol 1997; 168:1427-1434.

9. Wallengren N, Holtås S, Andrén-Sandberg $\AA$, et al. Rectal carcinoma: double contrast MR imaging for preoperative staging. Radiology 2000; 215:108-114.

10. Van der Molen A. Nefrogene systemische fibrose en de rol van gadoliniumcontrastmiddelen. Radiologie actueel 2007.

11. Brown G, Radcliffe A, Newcombe R, et al. Preoperative assessment of prognostic factors in rectal cancer using high-resolution magnetic resonance imaging. $\mathrm{Br} J$ Surg 2003; 90:355-364.

12. Brown G, Richards C, Newcombe R, et al. Rectal carcinoma: thin-section MR imaging for staging in 28 patients. Radiology 1999; 211:215-222.

13. Winter L, Bruhn $\mathrm{H}$, Langrehr J, et al. Magnetic resonance imaging in suspected rectal cancer: determining tumor localization, stage, and sphincter saving resectability at 3-Tesla-sustained high resolution. Acta Radiol 2007; 48:379-387.

14. Beets-Tan R, Beets G. Rectal cancer: review with emphasis on MR imaging. Radiology 2004; 232:335-346.

15. Koh D, Brown G, Temple L, et al. Rectal cancer: mesorectal lymph nodes at MR imaging with USPIO versus histopathologic findings-initial observations. Radiology 2004; 231:9199.

16. Harisinghani M, Barentsz J, Hahn P, et al. Noninvasive detection of clinically occult lymph node metastases in prostate cancer. N Engl J Med 2003; 348:2491-2499.

17. de Lussanet Q, Backes W, Griffioen A, et al. Dynamic contrast-enhanced magnetic resonance imaging of radiation therapy-induced microcirculation changes in rectal cancer. Int J Radiat Oncol Biol Phys 2005; 63:1309-1315.

18. de Vries $A$, Kremser $C$, Hein $P$, et al. Tumor microcirculation and diffusion predict therapy outcome for primary rectal carcinoma. Int J Radiat Oncol Biol Phys 2003; 56:958-965.

19. de Vries A, Griebel J, Kremser C, et al. Monitoring of tumor microcirculation during fractionated radiation therapy in patients with rectal carcinoma: preliminary results and implications for therapy. Radiology 2000; 217:385-391.

20. Ong K, Leen E. Radiological staging of colorectal liver metastases. Surg Oncol 2007; 16:714.

21. Wolberink S, Beets-Tan R, de Haas-Kock D, et al. Conventional CT for the prediction of an involved circumferential resection margin in primary rectal cancer. Dig Dis 2007; 25:8085.

22. Taylor A, Slater A, Mapstone N, et al. Staging rectal cancer: MRI compared to MDCT. Abdom Imaging 2006; 32:323-327.

23. Kulinna C, Eibel R, Matzek W, et al. Staging of rectal cancer: diagnostic potential of multiplanar reconstructions with MDCT. Am J Roentgenol 2004;183:421-427.

24. Bipat S, Glas A, Slors F, et al. Rectal cancer: local staging and assessment of lymph node involvement with endoluminal US, CT, and MR imaging-A meta-analysis. Radiology 2004; 232:773-783. 
25. Hartley A, Ho K, McConkey $\mathrm{C}$, et al. Pathological complete response following preoperative chemoradiotherapy in rectal cancer: analysis of phase II/III trials. $\mathrm{Br} \mathrm{J}$ of Radiol 2005; 78:934-938.

26. Bonnen M, Crane C, Vauthey J, et al. Long-term results using local excision after preoperative chemoradiation among selected T3 cancer patients. Int J Radiat Oncol Biol Phys 2004; 60:1098-1105.

27. Habr-Gama A, Perez R, Nadalin W, et al. Operative versus nonoperative treatment for stage 0 distal rectal cancer following chemoradiation therapy: long-term results. Ann Surg 2004; 240:711-717.

28. Toricelli P, Pecchi A, Luppi G, et al. Gadolinium-enhanced MRI with dynamic evaluation in diagnosing the local recurrence of rectal cancer. Abdom Imaging 2003; 28:19-27.

29. Rudisch A, Kremser C, Judmaier W, et al. Dynamic contrast-enhanced magnetic resonance imaging: a non-invasive method to evaluate significant differences between malignant and normal tissue. Eur J Radiol 2005; 53:514-519.

30. Capirci C, Rubello D, Chierichetti F, et al. Restaging after neoadjuvant chemoradiotherapy for rectal adenocarcinoma: role of F18-FDG PET. Biomed Pharmacother 2004; 58:451457.

31. Denecke T, Rau B, Hoffmann K, et al. Comparison of CT, MRI and FDG-PET in response prediction of patients with locally advanced rectal cancer after multimodal preoperative therapy: Is there a benefit in using functional imaging? Eur Radiol 2005; 15:1658-1666.

32. Capirci C, Rampin L, Erba $P$, et al. Sequential FDG-PET-CT reliably predicts response of locally advanced rectal cancer to neo-adjuvant chemo-radiation therapy. Eur J Nucl Med Mol Imaging 2007; 34:1583-1593.

33. Capirci $C$, Rubello D, Chierichetti $F$, et al. Restaging after neoadjuvant chemoradiotherapy for rectal adenocarcinoma : role of F18-FDG PET. Biomed Pharmacother 2004; 58:451457.

34. Cascani G, Avallone A, Delrio P, et al.18F-FDG PET is an early predictor of pathologic tumor response to preoperative radiochemotherapy in locally advanced rectal cancer. J Nucl Med 2006; 47:1241-1248.

35. Melton $G$, Lavely $W$, Jacene $H$, et al. Efficacy of preoperative combined 18Fluorodeoxyglucose Positron Emission Tomography and Computed Tomography for assessing primary rectal cancer response to neoadjuvant therapy. J Gastrointest Surg 2007; 11:961-969.

36. Young $\mathrm{H}$, Baum R, Cremerius $\mathrm{U}$, et al. Measurement of clinical and subclinical tumour response using [18F]-fluorodeoxyglucose and positron emission tomography: review and 1999 EORTC recommendations. Eur J Cancer 1999; 35:1773-1782.

37. Schiepers C, Haustermans K, Geboes K, et al. The effect of preoperative radiation therapy on glucose utilization and cell kinetics in patients with primary rectal carcinoma. Cancer 1999; 85:803-811.

38. van Ginkel R, Hoekstra H, Pruim J, et al. FDG-PET to evaluate response to hyperthermic isolated limb perfusion for locally advanced sift tissue sarcoma. J Nucl Med 1996; 37:984990.

39. Haberkorn U, Strauss L, Dimitrakopoulou A, et al: PET studies of fluorodeoxyglucose metabolism in patients with recurrent colorectal tumours receiving radiotherapy. J Nucl Med 1991; 32:1485-1490.

40. Kuriu $Y$, Otsuij E, Nakase $Y$, et al. Monoclonal antibody conjugated to gadolinium as a contrast agent for magnetic resonance imaging of human rectal carcinoma. J Surg Oncol 2006; 94:144-148.

41. Van Baardwijk A, Bosmans G, Boersma L, et al. PET-CT-based auto-contouration in nonsmall-cell lung cancer correlates with pathology and reduces interobserver variability in 
the delineation of the primary tumor and involved nodal volumes. Int J Radiat Oncol Biol Phys 2007; 68:771-778.

42. Daisne J, Duprez $T$, Weynand $B$, et al. Tumor volume in pharyngolaryngeal squamous cell carcinoma: comparison at CT, MR imaging, and FDG PET and validation with surgical specimen. Radiology 2004; 233:93-100. 



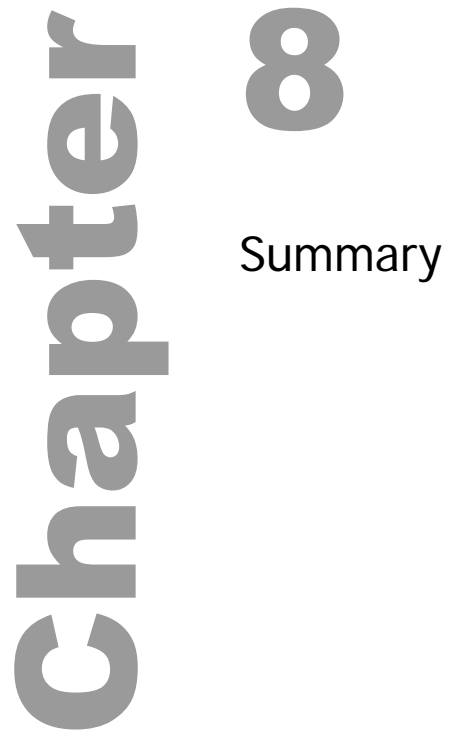




\section{Summary}

Chapter 2 gives a general overview of the role, performance, limitations and technique of Magnetic Resonance Imaging (MRI) in rectal cancer. In conclusion, MRI is worldwide accepted as the modality of first choice for the diagnostic work-up of rectal cancer patients.

The study presented in chapter $\mathbf{3}$ evaluates the value of gadolinium (Gd) enhanced T1-weighted MR images as a supplement to T2-weighted Fast Spin Echo (FSE) MR imaging sequences for the preoperative assessment of tumor invasion of the mesorectal fascia. The results showed that the addition of Gd enhanced T1-weighted images to T2-weigted FSE MR sequences did not improve the diagnostic accuracy of MRI.

An MR imaging protocol of T2-weighted FSE sequences alone performs well (Area Under the Receiver Operating Characteristic Curves=AUCs of 0.900.96), and therefore should be considered as standard for rectal cancer staging. Gadolinium enhanced sequences can be omitted. This saves acquisition time, costs and prevents nefrogenic systemic sclerosis, a disease which has been recently linked to the use of gadolinium contrast media in patients with renal insufficiency.

Chapter 4 evaluates the accuracy of Multi-Dector row Computed Tomography (MDCT) for the prediction of tumor invasion of the mesorectal fascia (MRF). Our data demonstrated a poor accuracy (54-66\%) of MDCT for the determination of tumor invasion of the MRF. Evaluation of the staging accuracy of MDCT at different tumor locations showed very poor staging results for the assessment of distally located rectal tumors (AUCS of $0.31-0.50$ ). This poor performance significantly improved for the determination of MRF tumor invasion of proximally located tumors (AUCs of $0.84-0.88$ ).

Our data demonstrate that MDCT can not be considered as a good alternative to MRI, especially not for staging distal tumors. Maybe one exception is when MRI is contra-indicated or unavailable and the tumor is expected to be located proximally in the rectum.

Chapter 5 assesses the performance of MRI for predicting tumor invasion of the mesorectal fascia (MRF) in locally advanced rectal cancer patients treated with chemoradiation therapy (CRT), and determines morphologic MR criteria for tumor invasion of the MRF after CRT.

Our data showed a high sensitivity of $100 \%$, a high negative predictive value of $100 \%$, but a moderate specificity $(32-59 \%)$ and a moderate positive predictive value (57-68\%) of postCRT MRI for the prediction of tumor invasion of the MRF. The moderate specificity appeared to be almost exclusively related to the limitation of postCRT MRI to differentiate between diffuse fibrotic tissue with and without small tumor nests, beyond any detection level of MRI. 
The results showed four different types of morphologic tissue patterns identified on MRI, associated with tumor invasion of the MRF at histology, in an upclimbing order from $0 \%$ to $90 \%$.

Our data demonstrate that despite limitations, postCRT MRI is able to identify patients with tumors that have regressed from the mesorectal fascia with a high negative predictive value. Based on the identified morphological MR patterns, surgeons get informed whether there is a high, intermediate or low risk to obtain a tumor positive circumferential resection margin when a resection plane is chosen along the mesorectal fascia. It is obvious that this is important information for planning the extent of the operation. Therefore, MRI is recommended as standard examination in the pre-operative work-up of rectal cancer patients undergoing long courses of CRT.

Chapter 6 evaluates the value of integrated 18F-fluorodeoxyglucose Positron Emission Tomography-Computed Tomography (PET-CT) for the prediction of tumor regression from the mesorectal fascia (MRF) in locally advanced rectal cancer patients treated with neo-adjuvant combined chemoand radiotherapy (CRT). For this purpose, patients were scanned with a PET-CT before and on average 6.3 weeks after CRT. The Standard Uptake Values (SUVmax) measured in the tumor volume and the shortest distance between the automatically outlined tumor volume and the mesorectal fascia were registered.

The results showed a statistical significant correlation between SUVmax changes and pathological tumor response expressed by tumor regression grades (TRG) which confirmed previous studies. This finding implies that chemoradiation induced tumor cell killing, expressed by TRG can be monitored by PET-CT. This may have important clinical implications as it suggests that adjustments of the neo-adjuvant therapy regime can be contemplated in non responders identified at an early stage by PET.

However, the pathologically verified tumor regression from the mesorectal fascia was not correlated with neither any tested SUV parameters nor with the shortest distance between the residual tumor and the mesorectal fascia. This suggests that PET-CT is not a useful tool for the evaluation of anatomical tumor changes in rectal tumors and therefore not accurate for the prediction of tumor regression from the mesorectal fascia. This suggests that the role of PET-CT as a planning tool for more conservative (less extensive) surgical resections is limited. 


\section{Samenvatting}

Rectumtumor is een vorm van kanker die zich afspeelt in het laatste deel van de dikke darm ook wel endeldarm genoemd. In de endeldarm verzamelt zich de ontlasting die via de anus het lichaam verlaat.

De behandeling van patiënten met een rectumtumor is afhankelijk van factoren die de prognose ongunstig beïnvloeden. Eén van de meest belangrijke factoren is de aanwezigheid van tumorgroei in de mesorectale fascie. Deze bindweefselplaat bakent het gebied af waarin het rectum gelegen is en dient als snijvlak voor de operatieve behandeling van tumoren gelegen binnen deze begrenzing. Deze vorm van operatieve behandeling wordt Totale Mesorectale Excisie (TME) genoemd en wordt in de regel voorafgegaan door een korte voorbestraling. Dit om het risico op een lokale terugkomst van de tumor in het bekken te beperken. Echter, deze behandeling is niet afdoende voor uitgebreidere tumoren met tumordoorbraak in de mesorectale fascie. De huidige trend is, om deze gevorderde tumoren agressief te behandelen met een combinatie van chemo- en radiotherapie voorafgaande aan de operatie. Het doel van deze agressieve voorbehandeling is een afname van het hoge risico op een lokale terugkomst van de tumor te bewerkstelligen. Daarnaast bestaat er in toenemende mate interesse voor een beperking van het operatiegebied, afhankelijk van de mate van reactie van de tumor (tumorrespons) op de agressieve voorbehandeling. Deze laatste trend wordt met name gestimuleerd door recent beschikbare effectieve combinaties van chemo- en radiotherapie die de kans op een tumorrespons aanzienlijk hebben doen toenemen.

Het spreekt voor zich dat voorafgaande aan de operatie een nauwkeurige voorspelling van de mate van tumoruitbreiding voor het bepalen van het type voorbehandeling en de mate van tumorrespons na voorbehandeling, ook wel stagering en re-stagering genoemd, noodzakelijk is voor een dergelijke behandelingsstrategie toegespitst op de individuele situatie van de patiënt. Het voorliggende proefschrift onderzoekt de rol die moderne beeldvormende technieken zoals Magnetische Resonantie Imaging (MRI), Multi Detector Row Computed Tomography (MDCT) en Positron Emission Tomography-Computed Tomography (PET-CT) in het kader van staging en re-staging zouden kunnen vervullen.

Hoofdstuk 2 beschrijft de rol van Magnetische Resonantie Imaging (MRI) in het behandelingstraject van patiënten met een rectumtumor. In dit hoofdstuk komen de nauwkeurigheid en beperkingen van deze techniek aan bod. Concluderend kan gesteld worden dat MRI thans wereldwijd wordt gezien als beeldvormende techniek van eerste keuze voor het bepalen van een behandelingsplan bij patiënten met een rectumtumor.

De studie in hoofdstuk 3 onderzoekt de aanvullende waarde van T1-gewogen MRI-afbeeldingen na intra-veneuze toediening van gadolinium contrastmiddel, toegevoegd aan een standaardprotocol van T2-gewogen Fast 
Spin Echo (FSE) MRI-afbeeldingen, voor het voorspellen van tumorgroei in de mesorectale fascie.

De resultaten van dit onderzoek tonen geen toegevoegde waarde aan van T1-gewogen MRI-afbeeldingen na gadolinium contrastmiddel. Een standaardprotocol bestaande uit alléén T2-gewogen FSE MRI-beelden geeft een nauwkeurige voorspelling van de tumorgroei in de mesorectale fascie, bespaart kosten en voorkomt potentiële complicaties samenhangend met gadolinium contrastmiddelen.

Hoofdstuk 4 onderzoekt de nauwkeurigheid van Multi-Detector row Computed Tomography (MDCT) voor het voorspellen van tumorgroei in de mesorectale fascie.

De resultaten van dit onderzoek tonen een Area Under the Receiver Operating Curves (AUC) van 0.62-0.71 aan, hetgeen een zeer matige nauwkeurigheid van deze techniek betekent voor het voorspellen van tumorgroei in de mesorectale fascie. De nauwkeurigheid van MDCT blijkt sterk af te hangen van de tumorlokatie in het rectum. De AUC voor het beoordelen van tumoren gelokaliseerd aan de voorzijde en het uiteinde van het rectum dichtbij de sluitspier, zogenaamde lage tumoren, bedraagt slechts 0.31-0.51. Echter, statistisch significante betere resultaten worden vastgesteld voor de beoordeling van tumoren gelokaliseerd aan het begin van het rectum hoog in het bekken, zogenaamde hoge tumoren ( $A U C=0.84-0.88$ ).

Dit betekent dat MDCT geen goed alternatief is voor MRI, de huidige techniek van eerste keuze voor de beoordeling van tumorgroei in de mesorectale fascie. Dit geldt met name voor lage tumoren aan de voorzijde van het rectum. Indien er een contra-indicatie bestaat voor het gebruik van MRI, bijvoorbeeld een pacemaker, vormen hoge rectum tumoren misschien een uitzondering op deze bevinding.

Hoofdstuk 5 onderzoekt de nauwkeurigheid van MRI voor het voorspellen van tumorgroei in de mesorectale fascie bij patiënten met gevorderde rectumtumoren die voorbehandeld zijn met chemo- en radiotherapie (CRT). In dit hoofdstuk worden tevens MRI-criteria bepaald voor het voorspellen van tumorgroei in de mesorectale fascie na voorbehandeling met CRT.

De MRI-resultaten tonen een hoge sensitiviteit (100\%), een hoge negatief voorspellende waarde (100\%) maar een matige specificiteit (32-59\%) aan voor het voorspellen van tumorgroei in de mesorectale fascie na CRT. Deze matige specificiteit is een gevolg van een op basis van MRI onmogelijk te maken onderscheid tussen littekenweefselvorming (ontstaan in het tumorgebied door de CRT) met tumorresten en littekenweefselvorming zonder tumorresten. Deze kleine tumorresten ingebed in littekenweefsel gaan in feite het onderscheidend vermogen van de MRI te boven.

Verder laten de resultaten van het onderzoek vier verschillende MRIpatronen van weefselinvasie zien die in opklimmende mate samenhangen met tumorgroei in de mesorectale fascie na CRT. Dit variërend van $0 \%$ tot $90 \%$. 
Onze studie laat ondanks ondervonden beperkingen van de MRI zien dat het mogelijk is om tumorregressie van de mesorectale fascie (tumorrespons waarbij de tumor zich terugtrekt van de mesorectale fascie) te voorspellen met een hoge negatief voorspellende waarde. Aan de hand van MRI-patronen worden chirurgen geïnformeerd of er een hoog, gemiddeld of laag risico bestaat op tumorgroei in de mesorectale fascie na voorbehandeling met CRT. Dit is een belangrijke bijdrage in de keuze te opereren volgens het TME principe langs de mesorectale fascie of een meer uitgebreide operatie. Het uiteindelijke doel van de chirurgische behandeling is immers een vrije (niet met tumor besmette) snijrand. Daarom is een MRI-onderzoek aan te bevelen bij iedere patiënt die voorbehandeld is met CRT.

Hoofdstuk 6 onderzoekt de waarde van Positron Emission Tomography-Computed Tomography (PET-CT) voor het voorspellen van tumorregressie van de mesorectale fascie (tumorrespons zodat de tumor zich terugtrekt van de mesorectale fascie) bij patiënten met gevorderde tumoren voorbehandeld met een combinatie van chemo- en radiotherapie (CRT). Voor dit doel wordt de SUVmax gemeten in het tumorvolume aan de hand van PETCT onderzoeken vóór en na CRT. De SUVmax (maximal Standard Uptake Value) is een maat voor de stofwisselingsactiviteit in de tumor. Verder wordt de afstand gemeten tussen het automatisch (met behulp van een computer programma) omlijnde tumorvolume en de mesorectale fascie op de PET-CT beelden na CRT. Deze metingen worden in verband gebracht met de bevindingen van het pathologisch anatomisch onderzoek van de operatiepreparaten.

De resultaten laten een statistisch significant verband zien tussen het percentage afname van de gemeten SUVmax en pathologische tumorrespons uitgedrukt in tumor regressie graden (TRG), hetgeen eerdere PET studies bevestigt. Dit betekent, dat de mate van tumorcel versterf ten gevolge van CRT, in feite uitgedrukt door de tumor regressie graad (TRG), voorspeld kan worden aan de hand van PET-CT. Deze bevindingen hebben mogelijk belangrijke klinische implicaties. Patiënten met slecht reagerende tumoren op CRT zouden in een vroeg stadium voor de operatie door PET-CT geïdentificeerd kunnen worden. Dit maakt een vroege aanpassing van het type CRT mogelijk.

Echter, de onderzoeksresultaten tonen geen statistisch verschil aan tussen patiënten met en zonder tumorregressie van de mesorectale fascie, zowel aan de hand van SUVmax metingen alsmede door middel van software die de tumor aflijnt. Dit suggereert dat in feite anatomische tumorregressie van de mesorectale fascie niet betrouwbaar kan worden voorspeld door middel van PET-CT. De rol van PET-CT als modaliteit voor het plannen van minder uitgebreide operaties voor patiënten met een goede reactie op CRT, is derhalve beperkt. 


\section{Dankwoord}

Een proefschrift is een product dat tot stand komt door teamwork. Een persoonlijk woord van dank wil ik graag richten tot:

Prof. dr. J.M.A. van Engelshoven: Beste Jos, je zag, zoals dat een echte hoogleraar betaamt, onmiddellijk de zwakke schakels in de artikelen die ik je aanbood, ook al lagen ze niet echt op het gebied van jouw interesse. Ik bewonder je kritische grondhouding en vooruitziende blik ten aanzien van het vakgebied Radiologie en dat heb je als leermeester goed overgebracht op je discipelen. Nu je dit leest ben je met pensioen. Geniet samen met Els van je welverdiende rust. Bedankt dat je me de kans gaf om radioloog te worden!

Dr. R.G.H. Beets-Tan, mijn GE-maatje: Beste Regina, je gaf mij het vertrouwen door mij op te nemen in je team. Je bent een belangrijke stimulans voor mijn wetenschappelijke ontwikkeling geweest en je hebt mij aangezet tot het schrijven van een proefschrift. I $\mathrm{k}$ ben blij dat je de erkenning voor je wetenschappelijke werk hebt ontvangen in de vorm van een hoogleraarschap. We blijven samenwerken. Bedankt!

Dr. G.L. Beets: Beste Geerard, ik heb je leren kennen als een vakman pur sang op alle fronten. Soepel, met humor en relativerend vermogen heb je mij, samen met Regina, door het promotietraject geloodst. Je bent niet alleen chirurg maar ook een beetje radioloog, een ijzersterke combinatie voor radiologisch research. Bedankt!

Drs. A.G. Kessels: Beste Fons, met mijn eigen statistische brouwsels heb ik je menig maal hoofdpijn bezorgd. Als een echte genie wist je altijd de vinger op de zere plek van het artikel te leggen. Ik ben er soms gek van geworden, maar je had meestal gelijk. Door je objectieve en waardevolle inbreng is ons waarschijnlijk veel commentaar van reviewers bespaard gebleven. Bedankt!

Dr. G. Lammering: Beste Guido, ik heb mij als een pitbull in je "vastgebeten" nadat ik je PET studiepopulatie op het spoor kwam. Je hebt mij een plek aangeboden in je oncologische research-groep waardoor ik een kijkje heb kunnen nemen in de Maastro high-tech keuken. Een must voor iedere oncologisch geïnteresseerd radioloog. Bedankt voor je aandeel in mijn proefschrift.

Drs. R.C. Dresen: Beste Elleke, je stage op onze afdeling was zeer welkom in een tijd dat mijn promotie en loopbaan in een stroomversnelling kwam. Je hebt ontzettend veel werk verricht. Dit heeft heeft er toe geleid dat je 
een aantal voordrachten en medeauteurschappen hebt verkregen. Het bleek dat je een fijne collega was. I $k$ wens je veel succes met je eigen promotie en opleiding.

Prof. dr. A.P. de Bruïne: Beste Adriaan, stiekem hoopt elke radioloog met beeldvorming de pathologie te kunnen evenaren. Voorlopig is dit echter nog niet het geval. Bedankt voor het werk dat je gedaan hebt en voor de fijne samenwerking in onze rectum-club.

Drs. A. Driesen: Beste Anne, waarschijnlijk ben je een beetje horendol van me geworden van alles wat ik wilde weten. Onderzoek roept nou eenmaal vele vragen op en die kun je niet allemaal beantwoorden met retrospectief materiaal. Bedankt voor de tijd die je gestoken hebt in dit proefschrift.

Drs. S.M. Engelen en Drs. M.J. Lahaye: Beste Sanne en Max, jullie waren altijd bereid mij met kleine dingetjes te helpen ook al had dat niet direct iets van doen met jullie onderzoek. Bedankt voor de fijne samenwerking in ons rectum-team. Succes met jullie promotie en opleiding.

Drs. B. van Houten: Beste Bart, we hebben flink gestoeid met de PET. Je hebt onze message met hand en tand verdedigd tijdens een grote boze plenaire sessie op de RSNA in Chicago. Bedankt voor je inzet en succes met je opleiding.

Dr. M.C. Oellers: Beste Michel, klinisch fysici zijn onmisbaar en ongekend populair in vakgebieden met veel high-tech. Jammer dat ik je heb moeten delen met anderen. I had namelijk nog zo veel vragen. Bedankt voor alles wat je hebt bijgedragen.

Drs. A.L. Arens: Beste Anne, je bent waarschijnlijk de enige (naast Wendy Schreurs) nucleair geneeskundige die precies de mesorectale fascie weet te lokaliseren. Je interesse en kennis van de Radiologie en een gezonde portie zeker niet SUVfe humor vormden een goede basis voor onze samenwerking. Bedankt.

Dr. A. Daniels-Gooszen: Beste Alette, in het jaar dat je bij ons was heb je een substantiële bijdrage geleverd aan onze afdeling, niet alleen in vrouwkracht, maar ook in de vorm van enthousiaste participatie in onderzoek. Ik hoop dat we in onze regio nog veel zullen samenwerken. Succes met je verdere carrière in de Eindhovense maatschap.

Dr. H.J. Rutten: Beste Harm, je patiëntenpopulatie heeft meer power gegeven aan een van de hoofdpublicaties van mijn boekje. Er ligt ongetwijfeld 
nog een schat aan informatie in Eindhoven te wachten die vraagt om gepubliceerd te worden. Bedankt voor de samenwerking en voor je gewaardeerde commentaar.

Drs. T.K. Oei: Beste Kiam, bedankt voor het altijd aanwezige enthousiasme om te participeren in het lopende rectumonderzoek.

Drs. A.A. van Baardwijk: Beste Angela, bedankt dat je ons op het idee bracht van de SBR.

Dr. R.J. Lamers: Beste Rob, je hebt mijn proefschrift van a tot $z$ gelezen. Bedankt dat je die moeite nam en mij hebt voorzien van waardevolle adviezen.

Etienne Lemaire: Als toenmalig onderzoekslaborant heb jij en uiteraard ook de rest van de MRI-groep veel betekend voor het lopende rectumonderzoek. MR-research begint namelijk met de acquisitie van de MRI data. Bedankt.

Ine Kengen: Bedankt voor de secretariële ondersteuning. Je hebt mij veel werk uit handen genomen en de puntjes op de i gezet.

Staf vakgroep Radiologie azM: Beste Riël, Ed, Jan, Michiel, Paul, BertJan, René, Dick, Wim, Simon, Elvier en Jan, het dagelijkse werk gaat gewoon door en dat zal toch door iemand gedaan moeten worden. Mede dankzij jullie kon er tijd voor mijn onderzoek vrijgemaakt worden. Bedankt.

Lei en Ria Quaedvlieg, mijn schoonouders: Beste Lei en Ria, bedankt voor jullie interesse in het wel en wee van een promoverende schoonzoon en Lei voor het onder de aandacht brengen van spelregels uit het groene boekje die ik blijkbaar al lang vergeten ben.

Huub en Dorothé Vliegen, mijn ouders: Lieve Huub en Dorothé, zonder jullie was ik er simpelweg niet geweest. Jullie hebben mij de mogelijkheid gegeven om te studeren hetgeen een belangrijke basis heeft gevormd voor mijn verdere leven. Bedankt voor jullie altijd aanwezige steun en liefde.

Mr. A.A.J. Quaedvlieg, mijn vrouw: Lieve Andrea, sinds ik je ken heb ik je zolder in beslag genomen en aldaar op zogenaamd 48b grote delen van de tijd doorgebracht. Ondanks dit ben je toch met me getrouwd. Ik hoop dat het niet tegenvalt nu ik van zolder afkom. Je bent waarschijnlijk de enige jurist die bekend is met de mesorectale fascie. I $k$ vond het erg knap dat je als leek een objectieve mening kon vormen en in staat was een bijdrage te leveren aan 
stukken die ik je heb laten lezen. Ik hoop dat er nu een tijd aanbreekt waarin we iets meer kunnen gaan genieten, van ons nieuwe huis en van ons in september te verwachten eerste kindje. Ik houd van je. 


\section{Curriculum vitae}

Roy Vliegen werd op 6 december 1969 geboren te Heerlen. In 1988 behaalde hij het Atheneum-B diploma aan het College Sancta Maria te Kerkrade. De studie Geneeskunde werd in 1996 afgesloten met het arts-examen. Tijdens zijn studie vervulde hij bestuurlijke functies en werd hij lid van het Medisch Genootschap SO.DA.NO.GO. Na het artsexamen was hij werkzaam als AGNIO Obstetrie en Gynaecologie in het Maasland Ziekenhuis te Sittard en aansluitend in het Academisch Medisch Centrum in hetzelfde vakgebied. In 1998 werd aangevangen met de opleiding Radiologie in het Academisch Ziekenhuis Maastricht onder leiding van Professor dr. Jos van Engelshoven. De opleiding werd in 2003 afgerond waarna hij staflid Radiologie in dit ziekenhuis werd. Sinds 2007 is hij lid van de maatschap Radiologie in het Atrium Medisch Centrum Parkstad. In 2006 is hij gehuwd met Andrea Quaedvlieg. 